\title{
WEAR TESTING OF CARPETS
}

\author{
By Herbert F. Schiefer
}

ABSTRACT

The NBS and Shawmut machines for testing the wear resistance of carpets, a gage for measuring the thickness of carpets, and an instrument for measuring the length of a tuft of pile from a carpet are described. The effects of the height of the vacuum-cleaner nozzle above the pile, the amount of suction at the nozzle, and the relative humidity and temperature of the air in which the test is made, on the results obtained with the NBS machine were studied. The effect of systematic changes in carpet construction on the wear resistance was investigated with each machine and under severe service conditions. Tests on 24 carpets of Axminster, velvet, and Wilton weaves were made on the two types of machines in several laboratories, and the results obtained in the different laboratories were correlated with each other and also with the results of 3 years of service tests on the same carpets. The correlations were highly significant, but large systematic differences between the weaves and between the laboratories were obtained. The latter difference is attributed primarily to a lack of uniformity in the testing procedures. Three types of vacuum-cleaning systems were used during the service tests, and their effect on the wear of the carpets and the effectiveness of cleaning were observed. The $S$ index, which is the time required to wear the pile of a carpet down to one-fourth of the matted pile thickness, corresponds closely with the time at which a carpet in a service test would be discarded on account of visible wear. The deviation of the $S$ index of a single test from the average is less than 10 percent in 9 trials out of 10 . The nature of the wear produced by the two machines on the pile fibers was found to be similar to that produced during the service tests. The analyses of carpets do not yield sufficient data to determine the probable durability of carpets in general. The relative wear of carpets for a given use can be evaluated by means of laboratory wear tests. For general interlaboratory comparison of the relative wear resistance of carpets, the testing machines and the testing procedures must be rigidly standardized and controlled.

\section{CONTENTS}

I. Introduction _.

II. Testing machines _...

1. NBS machine for testing the wear of carpets _._.

2. Shawmut machine for testing the wear of carpets _._.

3. Thickness gage for carpets _

4. Instrument for measuring the length of a tuft of pile $\ldots \ldots \ldots$

III. Testing procedures_._.

1. Analysis of carpet construction

2. Thickness _... 340

3. Wear of carpets

(a) NBS machine......... 340

(b) Shawmut machine

(c) Service tests

4. Wear indices

IV. Materials tested _.

V. Standardization of test on NBS machine

1. Effect of braking torque, downward force at each pulley, and speed of turntable........... 348

2. Effect of relative humidity and temperature

3. Effect of height of nozzle and suction at the nozzle $\ldots \ldots \ldots$ 
VI. Results

1. Wear indices

2. Service tests $\ldots \ldots \ldots \ldots \ldots$.

3. Comparison of service, NBS, and Shawmut tests on 24 carpets _ 357

4. Comparison of wear tests with analyses of carpets

5. Interlaboratory tests

6. Effect of type of vacuum-cleaning system on the service wear of carpets

7. Nature of wear of the fibers

8. Correlation of wear test results with analysis of carpets $\ldots \ldots \ldots 370$

9. Limitations of wear-testing machines _

VII. Summary _.......... 377

\section{INTRODUCTION}

After the publication of Research Papers RP315 and RP640, ${ }^{1}$ describing the NBS machine for testing the wear of carpets and discussing the results obtained with it, extensive wear-testing of carpets was undertaken in the laboratories of the carpet industry. Section IV on Pile Floor Coverings, of Subcommittee A-3, Committee D-13, American Society for Testing Materials, served as a clearing house for the discussion of these tests. It became apparent from these tests that there were some inconsistencies in the results obtained, between different laboratories and also within the same laboratory, on carpets of similar quality. It was found that the inconsistencies in the results could be attributed in a large measure to a lack of uniformity of production in carpet manufacturing. This information led to the early use of statistical control by a number of carpet manufacturers, following the method introduced by Shewhart. ${ }^{2}$ The results of the tests indicated also that some of the inconsistencies resulted from a lack of standardization of the wear-testing equipment, testing conditions, testing procedure, and method of evaluation of the results.

The investigation which forms the basis of this paper was undertaken in cooperation with Subcommittee A-3 to obtain information on the influence of a number of testing factors on the results and to shed much needed light on the interpretation of the results obtained in testing carpets tor wear. The improved NBS machine and the Shawmut machine for testing the wear resistance of carpets, a gage for measuring the thickness of carpets, and an instrument for measuring the length of a tuft of pile from a carpet are described. The results of laboratory tests of the wear of 24 carpets are compared with those obtained on the same carpets when subjected to 3 years of severe wear in actual service. Additional information is presented on the relationship between construction factors of carpets and the resistance to wear.

\section{TESTING MACHINES}

\section{NBS MACHINE FOR TESTING THE WEAR OF CARPETS}

The NBS machine for testing the wear of carpets is shown in figure 1. The specimen, circular in shape, is fastened to a metal turntable, 17 in. in diameter, shown in figures 2 and 3 . The turntable is placed

${ }^{1}$ H. F. Schiefer and A. S. Best, Carpet wear testing machine, BS J. Research 6, 927-936 (1931) RP315. Herbert F. Schiefer and Richard S. Celveland, Wear of carpets, BS J. Research 12, 155-166 (1934) RP640.

${ }^{2}$ W. A. Shewhart, Economic Control of Quality of Manufactured Product (D. Van Nostrand Co., Inc., New York, N. Y., 1931). 


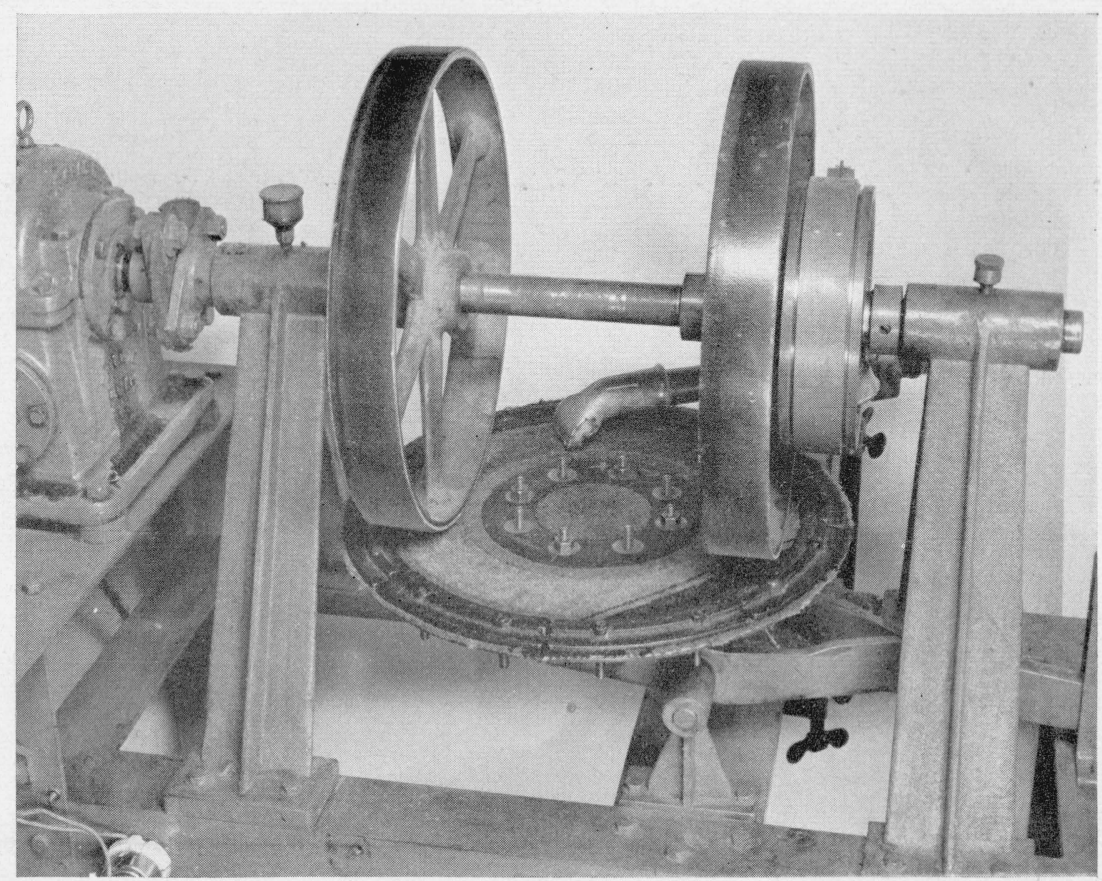

FiguRE 1.-The NBS machine for testing the wear of carpets.

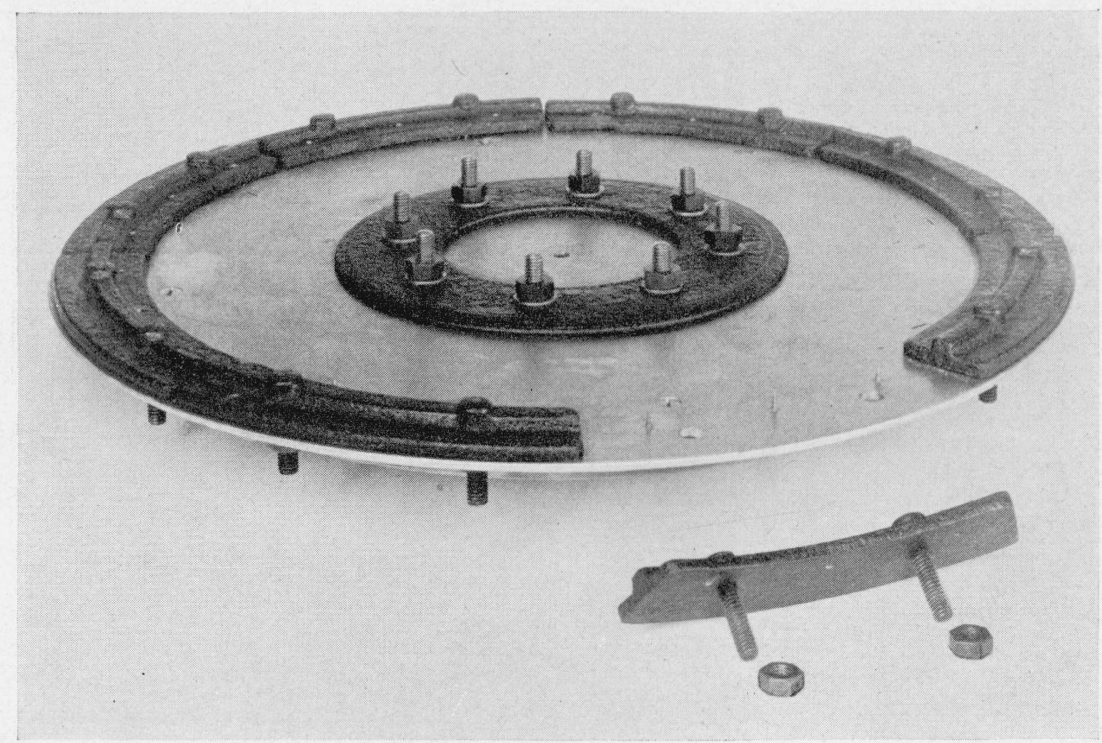

Figure 2.-Metal turntable. 


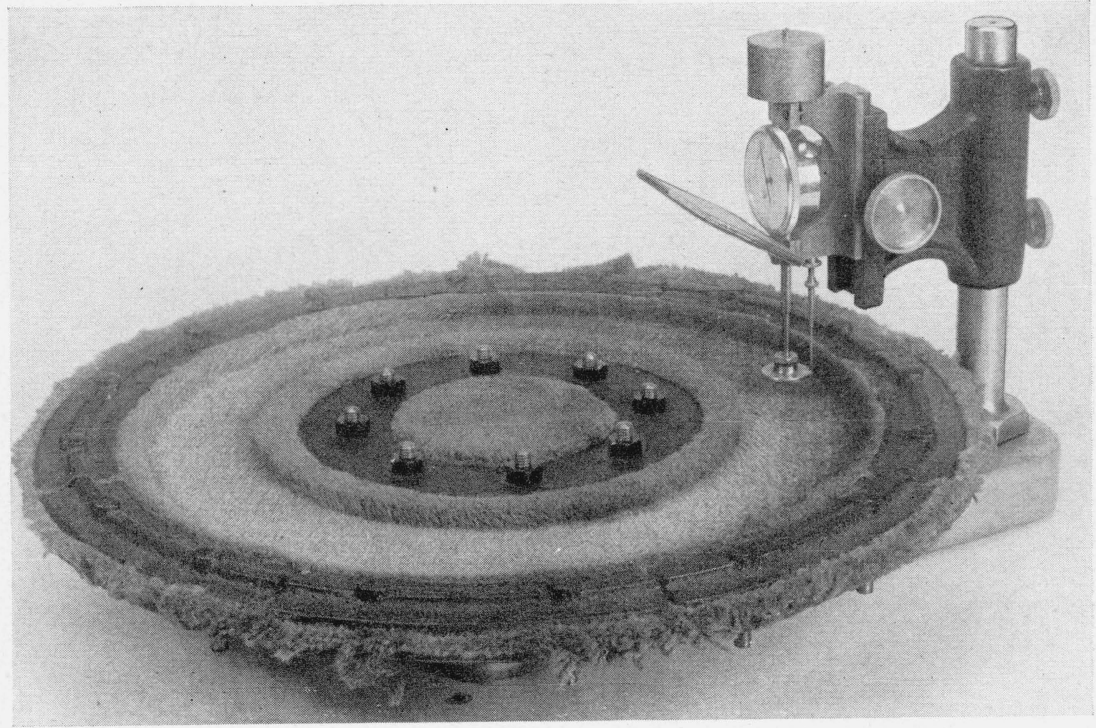

Figure 3.-Gage for measuring thickness of carpet mounted on turntable.

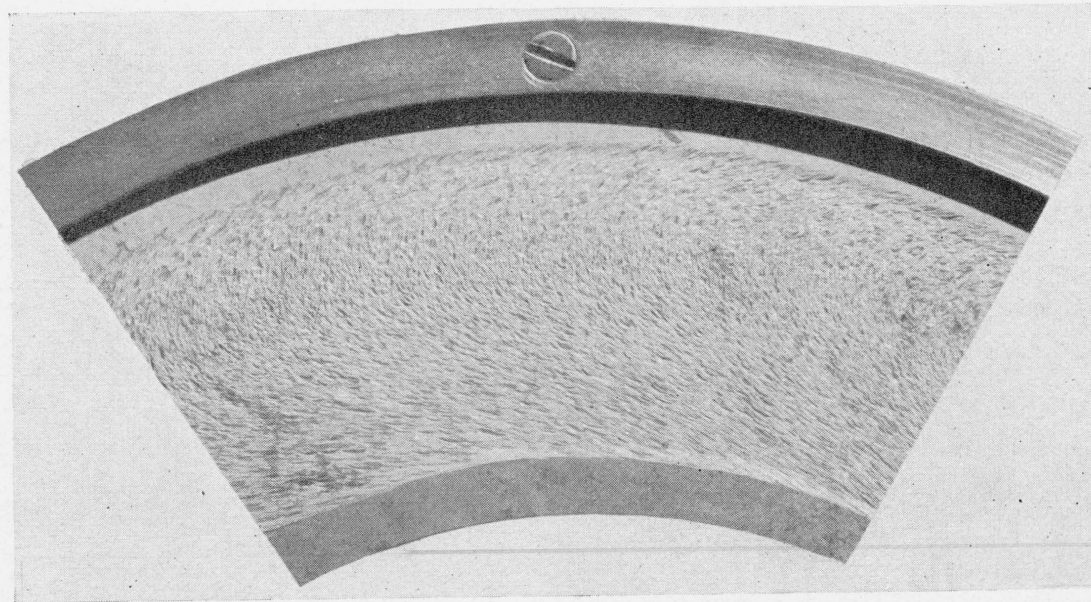

FIGURE 4.-Specimen of tile showing the twisting action produced by the NBS carpet-wear-testing machine. 


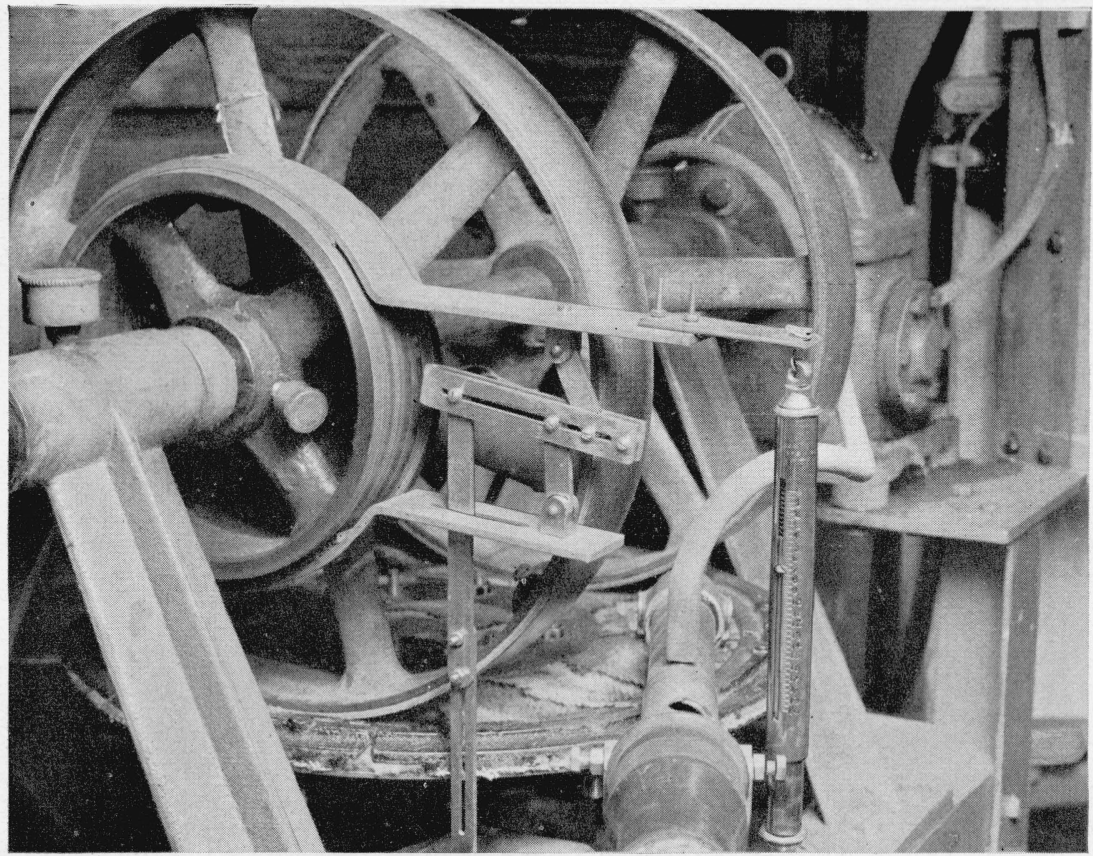

FiguRE 5.-Link-type brake for the NBS carpet-wear-testing machine.

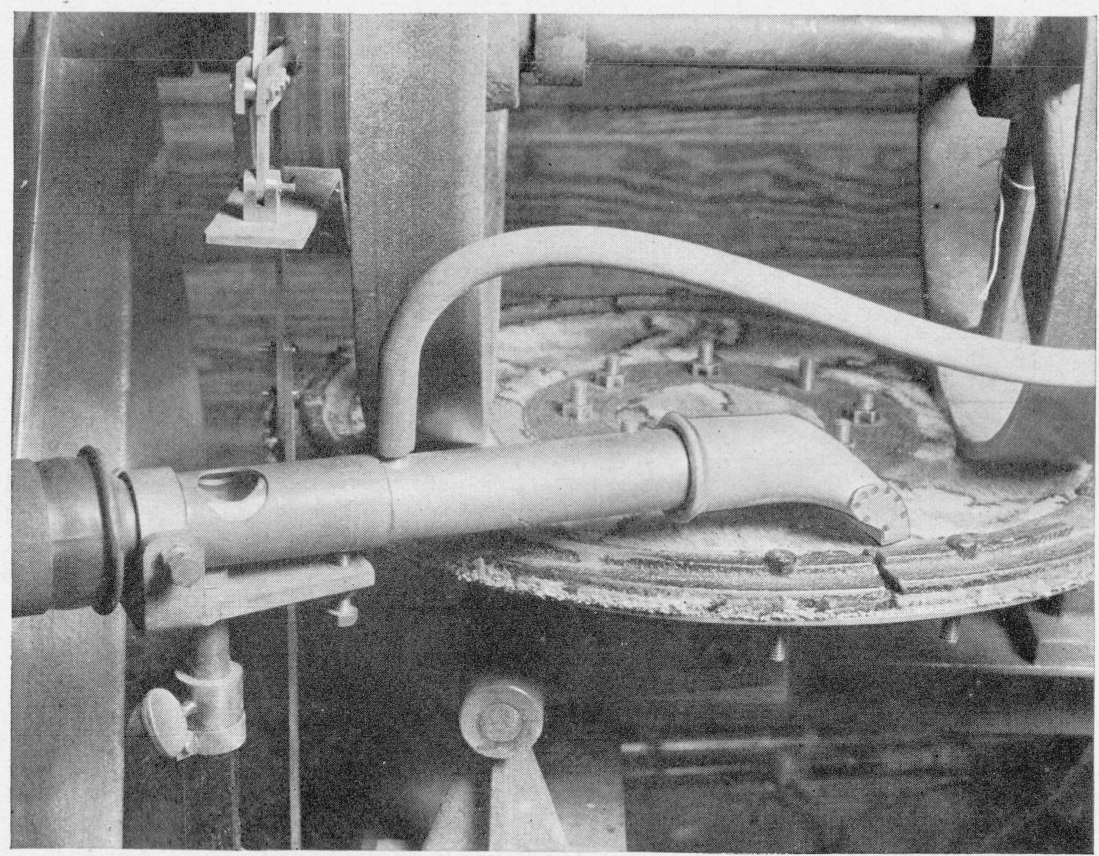

Figure 6.-Nozzle assembly of vacuum cleaner. 


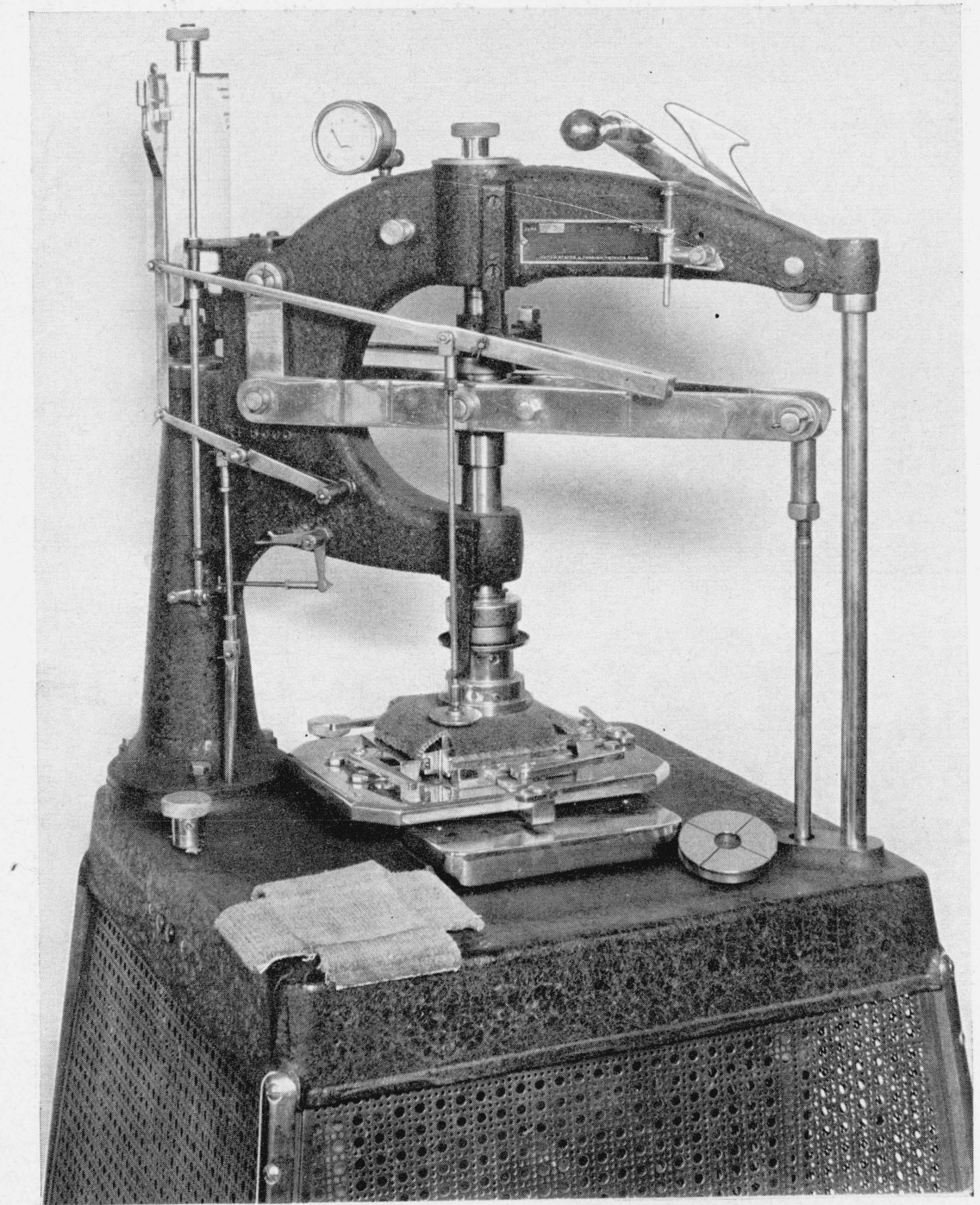

FIGURE 7.- Shawmut machine for testing the wear of carpet. 
over the end of a vertical shaft which is mounted in a special bearing at one end of a lever, so that when a weight is hung at the other end of the lever the carpet is brought to bear with the same vertical force against each of two leather-covered pulleys. The metal segments that are bolted to the turntable and the pointed pins which pierce the backing of the carpet specimen are effective in holding the specimen firmly during a test. The specimen is mounted with sufficient initial tension so that the exposed surface is flat and free from wrinkles. It is convenient to use a template, which exactly matches the turntable surface, in preparing the specimens for this test. The turntable is readily removed from the machine and may be transferred to a special gage (fig. 3) for measuring the thickness of the carpet specimen.

One of the pulleys which bears against the specimen during a test is driven by a shaft connected through a reduction gear to a motor. This pulley drives the turntable by friction. The other pulley, which is free to rotate on the driving shaft, is used as a brake to produce slipping of both pulleys on the carpet specimen as it rotates. A slight twisting action is produced on the pile of the carpet, because the carpet specimen is rotated in a plane perpendicular to the plane of rotation of the pulleys. This twisting action is shown qualitatively in figure 4 on a specimen of tile for floors. This specimen was tested with the driving pulley and using a coarse abasive in place of the leather. The markings on the surface of the specimen indicate the twisting action.

The load on the brake is indicated by the spring balance shown in figure 5. This load may be varied slightly by means of a wing nut at the bottom of the spring balance. The link-type brake ${ }^{3}$ shown in figure 5 has been found satisfactory for maintaining constant the load on the brake during a test. Large variations in the load on the brake can be obtained by changing the relative positions of the links. A Prony brake is also satisfactory, but requires manual adjustment and closer watching during operation.

The nozzle used in conjunction with a vacuum system to remove the worn material from the specimen during a test is shown in figure 6 . The nozzle opening is 2 by $1 / 4$ in. A connection to a manometer, located 9 in. from the opening of the nozzle, is provided to indicate the amount of suction. It was possible to maintain a suction of $12 \mathrm{~cm}$ $\mathrm{Hg}$ with the vacuum system when the opening of the nozzle was closed. The amount of suction can be varied by rotating the sleeve surrounding the nozzle tube. This rotation varies the amount of air which enters the nozzle tube through an opening of the sleeve. The mounting of the nozzle tube permits rotations about vertical and horizontal axes. The entire nozzle assembly can be displaced in a vertical direction. The rotation about the horizontal axis is controlled by a screw. The distance between the nozzle and the specimen can thus be easily adjusted and maintained at a constant value. This distance is measured by means of a plate, having the selected thickness, which can just be inserted between the pile and the bottom of the nozzle.

The distance between the centers of the two leather-covered pulleys is $11 \mathrm{in.}$ The width of each pulley is $2 \mathrm{in.} \mathrm{and} \mathrm{the} \mathrm{diameter} 15 \mathrm{in}$. The leather is vegetable-tanned belting leather, curried to contain $10 \pm 2$ percent of extractable oil. Each strip is $2 \frac{1}{4} \mathrm{in}$. wide, at least 48 in. long without any splices, and is cut from the butt center between 8

\footnotetext{
${ }^{3}$ This link-typ brake was designed by John Langton in the laboratory of Alex. Smith \& Sons Carpet Co., Yonkers, N. Y.
} 
and $12 \mathrm{in.}$ from the backbone. The flesh side is leveled by machine, so that the average thickness of the piece is $0.205 \pm 0.005$ in. An endless belt is made of each strip to fit very snugly on the pulley. The leather is fastened to the pulley with fish glue. The glue is applied to the flesh side of the leather and to the pulley surface, which should be rough. The pulley surface should be clean, dry, and hot when the glue is applied. This can be accomplished by immersing the pulley in boiling water for 15 minutes just prior to mounting the leather. After the glue is dry, the pulley is mounted in a lathe and the leather is turned down to a width of 2 in. and an over-all diameter of pulley and leather of $15^{5 / 16}$ in. The surface is then polished with fine sandpaper. The machine should be operated for at least 100,000 revolutions of the turntable, using any available carpet specimen that will not wear out during this period, to produce a highly polished surface on the leather. This highly polished surface is retained for several million revolutions of the turntable during normal testing.

The speed of the driving shaft is constant in the NBS testing machine, and depends upon the speed of the motor and the ratio of the reduction gear. The speeds of the turntable and braking pulley depend upon the speed of the driving pulley, the frictional forces between the leather and the carpet specimen, and upon the braking torque. The speed of the driving pulley and the braking torque can be maintained constant. The frictional force between the carpet specimen and the pulleys may vary, however, with different carpets and during a test of the same specimen. The relationship between the speeds of the pulleys and the turntable, for a considerable range of speeds of the driving pulley, is such that the amount of slippage at the center of the driving pulley relative to the carpet specimen remains very nearly equal to the amount of slippage at the center of the braking pulley relative to the carpet specimen. The slippages at the centers of the two pulleys relative to the carpet specimen are in opposite directions and range between 9 and 13 percent during normal testing.

In a series of tests for which the braking torque was $7.5 \mathrm{lb} \mathrm{ft}$ and the speed of the driving pulley $64 \mathrm{rpm}$, the speed of the turntable and of the braking pulley had the frequency distributions given in table 1. It was also found that the speeds of the two pulleys and of the turntable are related linearly to each other over a 20-percent change in speed of the driving pulley. In other words, for a given increase in speed of the turntable, the percentage increase in the speed of the driving pulley is equal to the percentage increase in speed of the braking pulley. If the speed of the driving pulley and the braking torque are maintained constant during a test, it is possible that the frictional force between the carpet and the leather of the pulleys may change owing to a change in the fibers of the pile of the specimen and in the surface of the leather. If the frictional force is increased, then the speeds of the turntable and of the braking pulley are automatically increased and the amount of slippage between the pulleys and the pile of the carpet specimen is decreased. The energy expended on the specimen per revolution of the turntable probably remains very nearly constant for these conditions. This constant expenditure of energy per revolution of the turntable probably explains why no marked increase in temperature of the leather and of the pile of the specimen has been observed in any of the carpets tested on this type of machine. 
TABLE 1.-Frequency distribution of the speeds of the turntable and braking pulley when the speed of the driving pulley and the braking torque were maintained at 64 rpm and 7.5 pound-feet, respectively

\begin{tabular}{|c|c||c|c|}
\hline \hline \multicolumn{2}{|c|}{ Turntable } & \multicolumn{2}{c|}{ Braking pulley } \\
\cline { 1 - 2 } Speed & Frequency & Speed & Frequency \\
\cline { 2 - 3 } & & & \\
\cline { 3 - 4 }$r p m$ & & $r p m$ & \\
78 & 1 & 47 & 1 \\
79 & 10 & 48 & 0 \\
80 & 25 & 49 & 1 \\
81 & 16 & 50 & 11 \\
83 & 6 & 51 & 29 \\
& 1 & 52 & 15 \\
& & 53 & 2 \\
\hline
\end{tabular}

\section{SHAWMUT MACHINE FOR TESTING THE WEAR OF CARPETS}

The Shawmut machine for testing the wear of carpets is shown in figure 7 . The test specimen is $6 \frac{1}{2}$ in. square. A square $1 \frac{1 / 2}{2}$ inches

$3.5^{\prime \prime}$

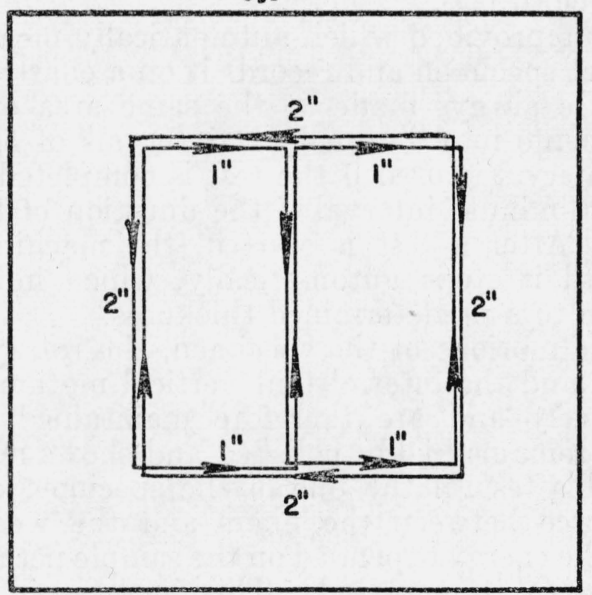

FIGURE 8.-Direction and magnitude of the transverse motions of the specimen during a test on the Shawmut machine.

on a side is cut from each of the four corners of this specimen. The resultant specimen, also shown in figure 7 , contains a central square,

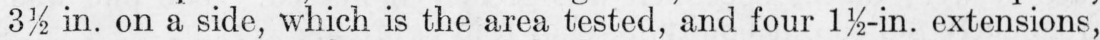
one on each side of this square, which are used to fasten the specimen in the special clamps of the machine. The clamps are provided with means for keeping the specimen under tension throughout the test. The clamps and the horizontal platen over which the specimen is stretched are mounted on two horizontal plates, each plate being actuated by a special cam to produce alternate transverse motions at right angles to each other. These motions follow a definite sequence which is repeated after two cycles. The directions and magnitudes of these motions for the two cycles are indicated in figure 8, where the indicated trace shows the motion of the center of the wearing surface relative to the specimen. These motions bring the various 
sections underneath the wearing surface, which is parallel to the specimen. This wearing surface, also shown in figure 7 , is annular in shape, $2 \frac{3 / 4}{4}$. outside diameter and $3 / 4$ in. inside diameter. It is made of special metal and is very smooth. It contains four radial slots, which are at right angles to each other. The wearing surface is given a reciprocating rotary motion about the vertical axis of the annular disk. The disk is brought to bear against the carpet specimen with a constant force exerted by a dead weight through a lever. A cam lifts the disk from the carpet specimen intermittently. The pile of the specimen is thus subjected to slipping, twisting, and compressive forces which continually change in direction and magnitude, but repeat regularly during the test. The motions of the edges of the radial slots in the wearing surface relative to the pile produce the wear on the specimen. A typically worn-out specimen is also shown in figure 7 .

The worn-off material is removed from the specimen during a test by suction, which is applied through the central hole of the wearing disk. The amount of suction is indicated on a gage and may be adjusted to a predetermined value by means of a valve. The debris is collected in a cloth bag.

A mechanism is provided which automatically measures the thickness of the carpet specimen and records it on a chart. The thickness measurements are always made at the same area of the specimen. These measurements may be made at intervals of 5 or 20 minutes. The 5-minute interval is used if the test is completed in less than 15 hours and the 20-minute interval if the duration of the test is more than 15 hours. After a test is started, the machine runs without interruption until it stops automatically, which indicates that the specimen is worn to a predetermined thickness.

The transverse motions of the specimen, the rotary motion of the wearing surface, and the intermittent vertical motion of the disk are produced positively and are therefore maintained constant. The wearing surface remains highly polished and shows no change during the tests. In this test, if the pile of the specimen changes so that the frictional force between the fibers and the wearing surface is increased, then the energy expended on the sample per rotary motion of the wearing surface is increased. This increase in expenditure of energy for each rotary motion of the wearing surface probably explains the excessive heating of the wearing disk during the tests of some of the carpets. This condition usually produced a shimmy motion between the specimen and the wearing surface, and the resultant rate of wear of the specimen was greatly increased. The pile was frequently pulled out bodily, as shown in figure 9 .

\section{THICKNESS GAGE FOR CARPETS}

The thickness gage for measuring the wear of carpets is shown in figure 3. It is provided with a base on which the turntable of the wear-testing machine is mounted, so that the ceiter of the worn track of the specimen is located directly underneath the 1-in. circular foot of the dial micrometer. The foot is fastened to the stem of the dial micrometer with a ball-and-socket connection. A weight is placed on top of the stem to make the pressure which the foot will exert on the specimen equal to $0.75 \mathrm{lb} / \mathrm{in}^{2}$. The dial-micrometer assembly 


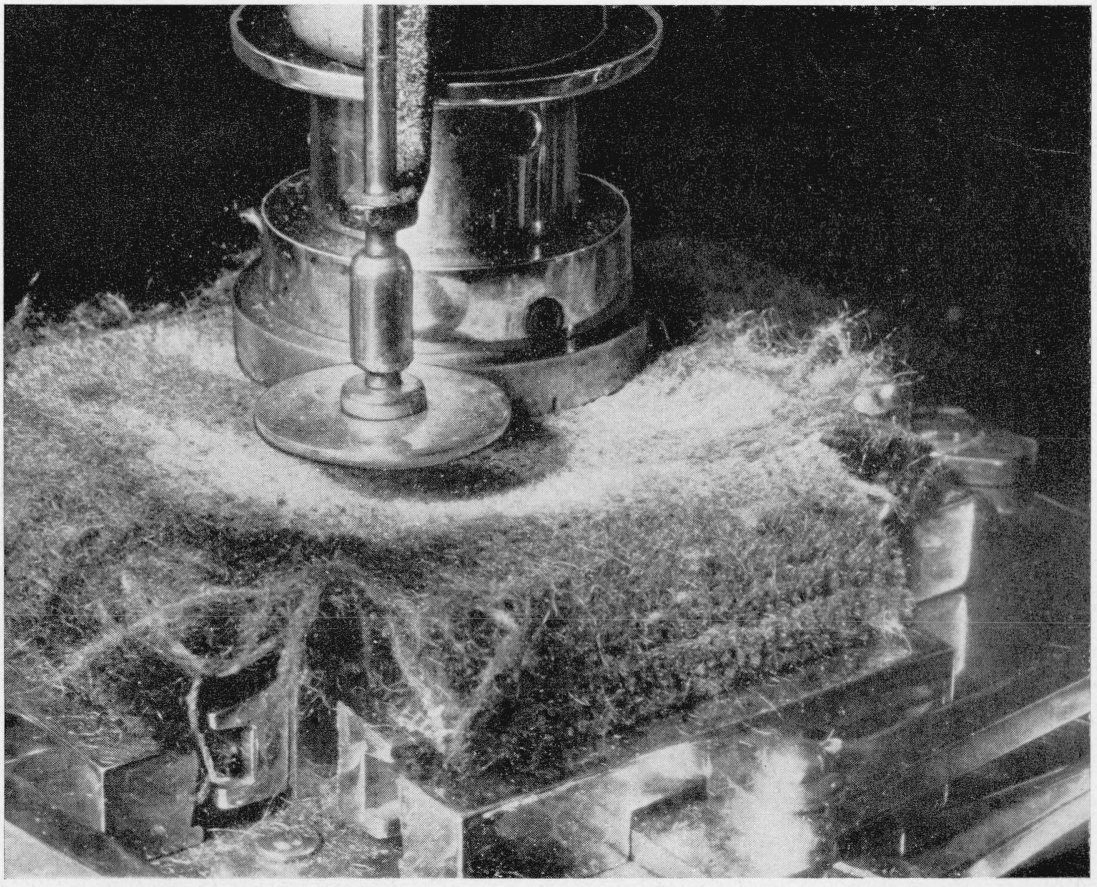

Figure 9.-Appearance of specimen which became excessively heated during a test on the Shawmut machine. 


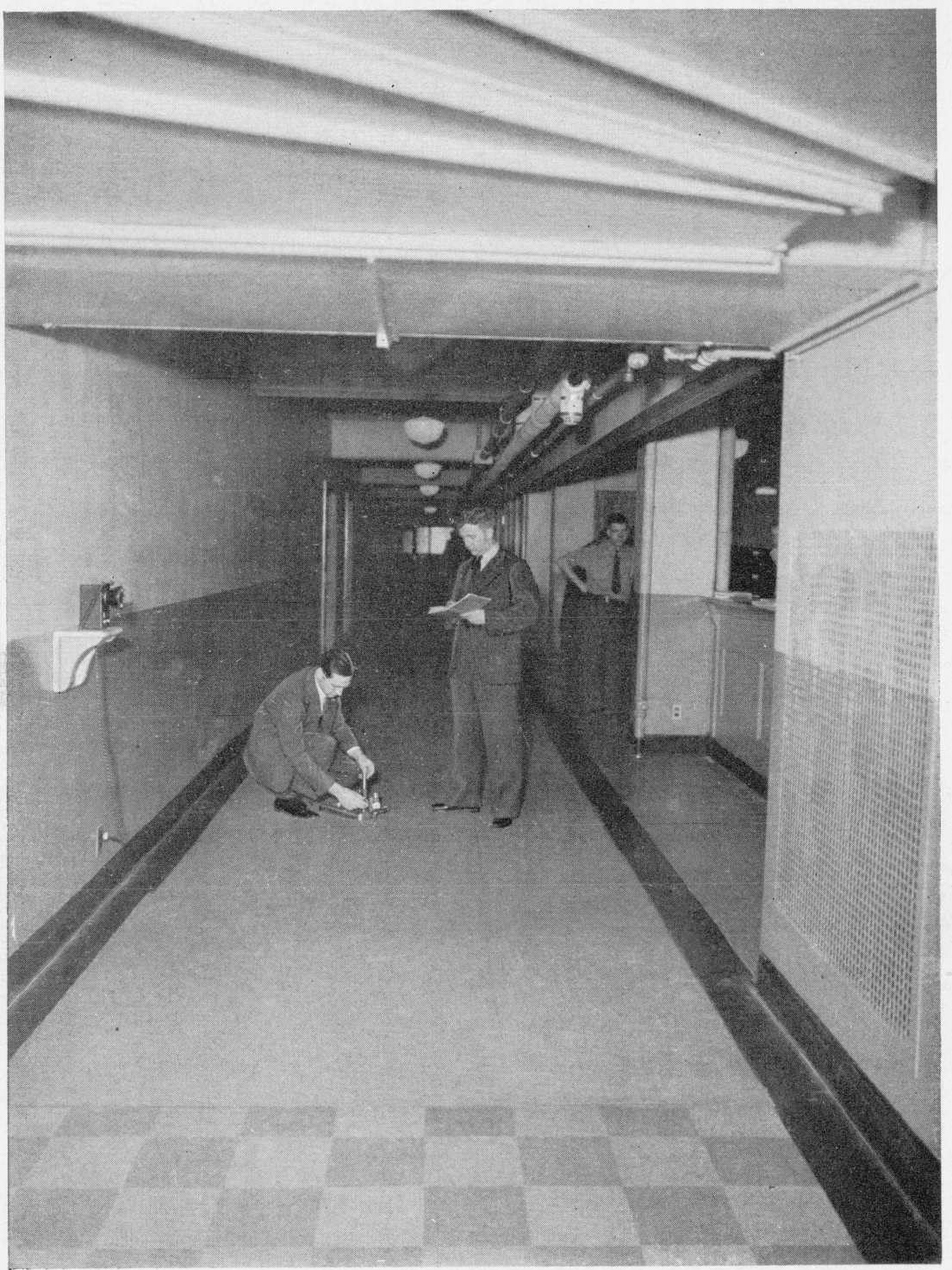

FIGURE 10.-Gage for measuring the thickness of the carpets laid in a long corridor for service wear tests. 


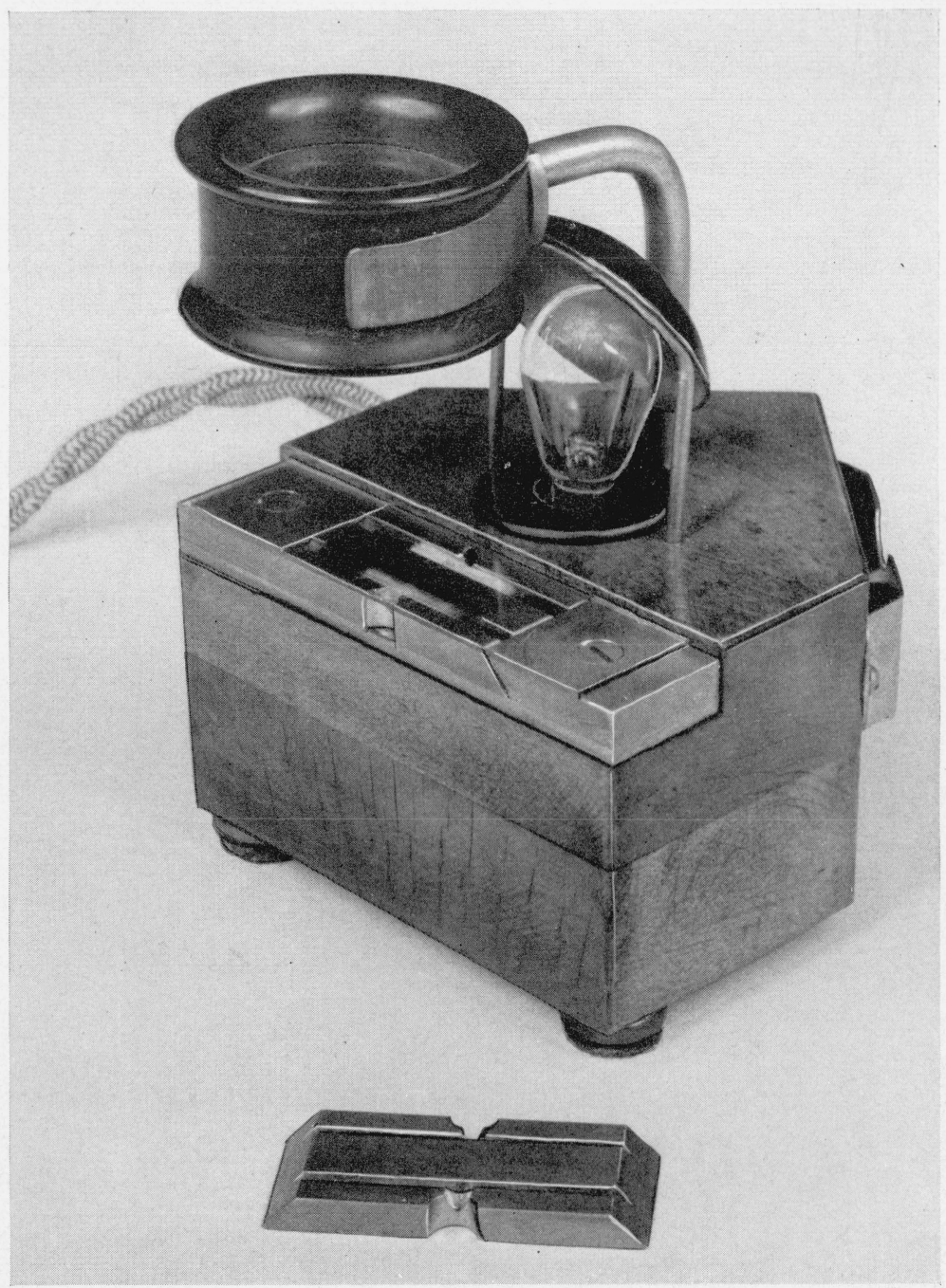

FiguRE 11.-Instrument for measuring the length of a tuft of pile. 
may be moved vertically by means of a rack and pinion. A needle is fastened to this assembly. The point of the needle is in the plane of the bottom surface of the foot when the pointer of the dial micrometer coincides with the zero of the scale. It is apparent that when the dial-micrometer assembly is lowered, the foot will rest on the specimen and exert a pressure of $0.75 \mathrm{lb} /$ in. $^{2}$ and the needle will pierce the carpet until the point comes in contact with the steel plate of the turntable. The distance between the point of the needle and the bottom surface of the foot is equal to the thickness of the carpet. This value is read directly from the dial to the nearest $0.001 \mathrm{in}$. The turntable may be rotated and the thickness measured at a number of positions around the worn track.

The entire gage mounting may be rotated through $180^{\circ}$, and the thickness of carpets which are laid on hard-surfaced floors may be measured. Figure 10 shows the instrument being used for thickness measurements of carpets laid in a long corridor. A mirror mounted in front of the dial greatly facilitates reading of the scale when these thickness measurements are made.

\section{INSTRUMENT FOR MEASURING THE LENGTH OF A TUF'T OF PILE}

The instrument for measuring the length of a tuft of pile yarn taken from a sample of carpet is shown in figure $11 .{ }^{4}$ The instrument is provided with two in terchangeable brass plates. Each plate contains two $\mathrm{V}$-shaped slots. The base angle of each slot is $60^{\circ}$. The four slots are of different sizes, the widths across the top equalling $0.150,0.125,0.100$, and 0.050 in., respectively. The largest slot is used for measuring very coarse pile yarns and the smallest slot for measuring very fine pile yarns or the plies of a coarse pile yarn. The other two slots are for yarns of intermediate sizes. A plate is inserted in the instrument with the slot of appropriate size in the front position, directly underneath a wide-field lens having a magnification of about 5 diameters. A glass plate on which is reproduced a scale, $1.5 \mathrm{in}$. long and graduated in 0.01 in., fits over the slot. The scale is on the bottom surface of the glass plate to minimize the effect of parallax. The scale is illuminated by a small lamp. The U-shaped tuft to be measured is picked up with tweezers at the bottom of the $U$, the glass scale is tilted up at the front, and the two ends of the tuft are inserted into the $V$-slot. The tuft is readily straightened by applying a slight force with the tweezers. The glass scale is lowered, which further straightens the tuft and presses it slightly into the $V$-slot. The tuft is then released, and the tweezers are withdrawn through a slot and semicircular recess at the front of the plate. The scale is moved horizontally to aline the zero line of the scale with one end of the tuft. The line of the scale which coincides with the other end of the tuft is noted, and the length is recorded to the nearest 0.01 inch. With this instrument the lengths of 100 tufts can be measured in 1 hour with ease. The standard error of 50 measurements was found to be 0.002 inch.

\footnotetext{
${ }^{4}$ A similar instrument has been described by Hopkins and Dow in Textile World 87, 1159 (1937).
} 


\section{TESTING PROCEDURE}

\section{ANALYSIS OF CARPET CONSTRUCTION}

The number of rows of tufts per inch of length of carpet was determined by counting the rows in 10 in. at three different places, and the pitch, number of tufts per inch of width, was determined by counting the tufts in 10 in. at three different places. The pile height, length of the pile tuft projecting above the back, was measured with a steel scale graduated in $1 / 64$ in. The average of five measurements was taken as the pile height. The tuft length was measured with the instrument described in section II, 4. The average of 50 measurements was taken as the tuft length. These 50 tufts were weighed, and the weight per tuft and the weight of the pile yarn per inch of length were computed. The density of the pile was computed by the expression $2 R P W / L$, where $R$ is the number of rows per inch, $P$ is the pitch per inch, $W$ is the weight per tuft in grains, and $L$ is the length of the tuft in inches.

\section{THICKNESS}

The thickness of the pile of a carpet was obtained by subtracting the thickness of the back of the carpet from the total thickness.

To measure the thickness of the back, a 6 - by 10 -in. specimen was taken, a flame was directed over the area of the specimen, and the pile was destroyed by alternate charring and brushirg without damage to the back. The average of 10 measurements with the instrument described in section II, 3, was taken as the thickness of the back.

The total thickness of a carpet was obtained by measuring a specimen after it was mounted on the turntable. The average of 10 measurements, made at different places around the annular area to be tested for wear, was taken as the total thickness of the carpet. The total thickness and pile thickness of a specimen after it had been subjected to wear on the wear-testing machine were measured in like manner.

\section{WEAR OF CARPETS}

(a) NBS MACHINE

Unless otherwise noted, the wear tests on the NBS machine were made under the following conditions:

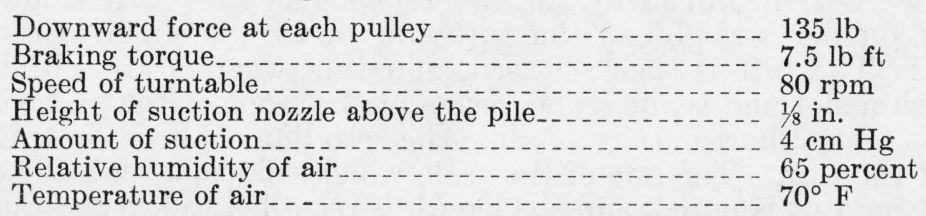

The thickness of the carpet specimen was measured at the beginning of a test and again after $500,1,000,2,000,5,000,10,000$, and after every additional 10,000 revolutions of the turntable. The thickness of the pile was plotted against the number of revolutions of the turntable. A test was discontinued when the plot showed that the pile was worn off. Two or more specimens of each carpet were tested. 
A chart was mounted on the recording drum and reference marks corresponding to zero thickness were recorded. The machine was set to stop when the thickness was equal to the back thickness, previously determined on a specimen of the same carpet. The specimen, already described, was mounted in the machine and its thickness was recorded. The machine was started and the suction was adjusted until the gage indicated a suction of 20 in. of water. ${ }^{5}$ The thickness was recorded at regular intervals. The test was continued without interruption until the pile was worn off and the machine automatically stopped.

\section{(c) SERVICE TESTS}

The service tests on 24 carpets of different constructions were conducted in the south corridor located on the fifth floor of the Procurement Division Building, Washington, D. C. This location was selected because several thousand people walked through this corridor each day. Each carpet consisted of three strips $27 \mathrm{in}$. wide and $5 \mathrm{ft}$. long (see fig. 10). The three strips were sewed together to cover the width of the corridor. The 24 carpets were sewed together to form a strip $120 \mathrm{ft}$. long. A 5 -ft. length of a spare carpet was sewed to each end to absorb any excess wear occasioned at the ends. The carpets were laid in one continuous length over a fairly hard-surfaced floor of asphalt tile. The carpet strip was tacked directly to the floor around the edges. The thicknesses of the carpets were measured on the floor at the beginning of the test and at regular intervals during the test. Thirty measurements were made on each carpet construction. These measurements were spaced approximately $1 \mathrm{ft}$. along the length and across the width of each carpet. A photoelectric cell counter was installed to count the number of persons walking over the carpets. each day. The carpets were cleaned once every day, using the central suction system of the building. The duration of each cleaning period was approximately 45 minutes. Detached vacuum-cleaning units of the rotating brush and agitator types were used during a portion of the duration of the service tests. The amount of dirt removed each day by each of the three cleaning systems was weighed during a test period lasting about 6 months. The service tests were continued for 162 weeks, during which time about 2,000,000 people walked over the carpets. The carpet strips were then washed in a commercial carpetcleaning plant and each carpet was photographed.

\section{WEAR INDICES}

The change in thickness of the pile of a carpet during a test has been found to be the best measure of the amount of wear. The curve shown in figure 12 is typical of the change in thickness of the pile that takes place during a laboratory or a service-wear test. The thickness of the pile decreases very rapidly during the early stage of a test. This rapid decrease is caused primarily by a matting of the pile. After the pile has been matted, its thickness decreases at a fairly uniform rate for the remainder of the wear test.

The wear index used in Research Paper RP640, ${ }^{6}$ referred to as the $K$ index, is defined as the number of thousands of revolutions of the

\footnotetext{
5 A mercury manometer probably would perform more satisfactorily than the gage furnished with the machine.

${ }^{6}$ See footnote 1 on p. 334.

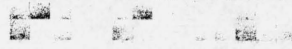


turntable required to wear the pile down to one-fourth of its original thickness. This point is marked $K$ in figure 12 . It was believed by some members of the carpet industry that the $K$ index did not evaluate all weaves of carpets in the correct relationship, particularly the lower-quality carpets of the Axminster weave. To overcome this criticism, the author suggested a new wear index, referred to as the $S$ index, which is based upon the matted thickness of the pile. The matted thickness is obtained by extrapolating the more or less flat portion of the curve back to the zero of the abscissa scale, shown by

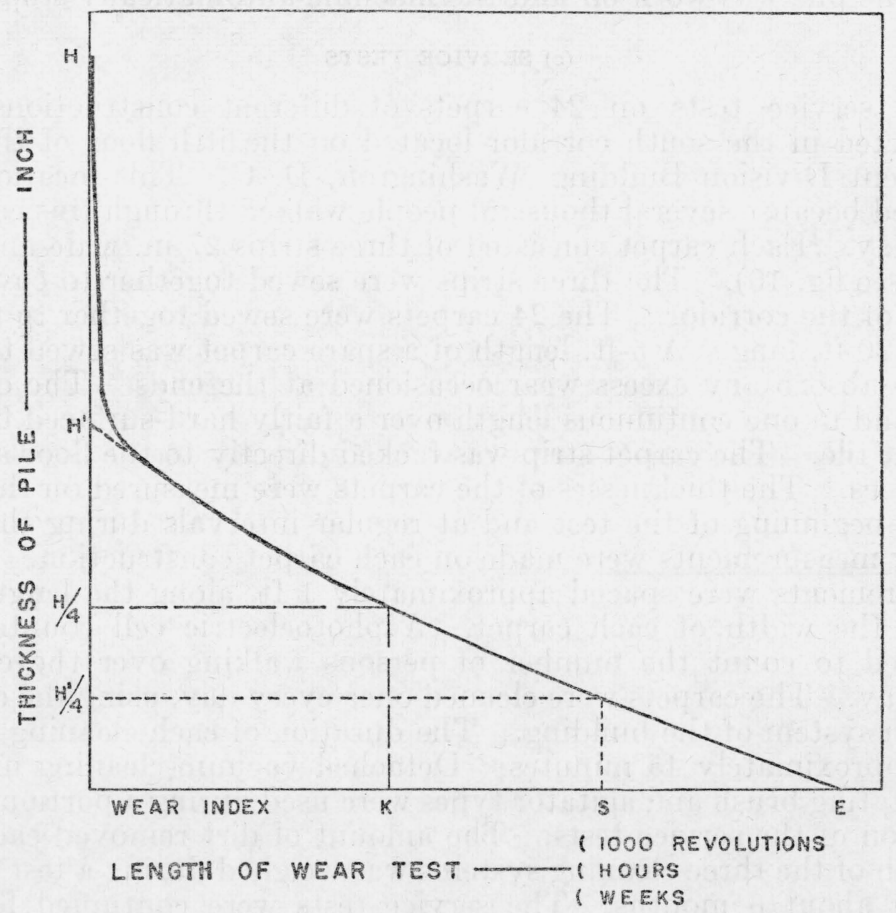

FIGURE 12.-Typical curve showing the change in thickness of the pile of a carpet during a wear test.

the broken line in figure 12. The $S$ index is defined as the number of thousands of revolutions of the turntable required to wear the pile down to one fourth of its matted thickness. This point is marked $S$ in figure 12. Another wear index which has been proposed is the number of thousands of revolutions of the turntable required to wear the pile down to zero thickness. This wear index is known as the $E$ index and is marked $E$ in figure 12. The unit for these wear indices obtained with the Shawmut machine is expressed in hours and the unit for these wear indices obtained in the service tests is expressed in weeks. 
IV. MATERIALS TESTED

The carpets for this investigation were furnished by the Bigelow Sanford Carpet Co., the Mohawk Carpet Mills, Inc., and the Alexander Smith \& Sons Carpet Co. Some of the carpet series were especially woven according to specified constructions. Several carpets from miscellaneous sources were added to the list. The carpets and pertinent information regarding their construction are listed in table 2 . 
TABLE 2.-Analysis of carpets and test results

\begin{tabular}{|c|c|c|c|c|c|c|c|c|c|c|c|c|c|c|}
\hline \multirow{2}{*}{ Weave A } & \multirow{2}{*}{$\begin{array}{c}\text { Rows } \\
\text { per } \\
\text { inch, } \\
R\end{array}$} & \multirow{2}{*}{$\begin{array}{c}\text { Pitch } \\
\text { per } \\
\text { inch, } \\
P\end{array}$} & \multirow{2}{*}{$\begin{array}{c}\text { Weight } \\
\text { per } \\
\text { tuft, } \\
W\end{array}$} & \multirow{2}{*}{$\begin{array}{c}\text { Length } \\
\text { of } \\
\text { tuft, } \\
L\end{array}$} & \multirow{2}{*}{$\begin{array}{l}\text { Weight } \\
\text { of tuft } \\
\text { per inch } \\
\text { length } \\
B=W / L\end{array}$} & \multirow{2}{*}{$\begin{array}{c}\text { Height } \\
\text { of } \\
\text { pile,b } \\
H\end{array}$} & \multirow{2}{*}{$\begin{array}{l}\text { Initial } \\
\text { thick- } \\
\text { ness } \\
\text { of } \\
\text { pile。 }\end{array}$} & \multirow{2}{*}{$\begin{array}{c}\text { Initial } \\
\text { thick- } \\
\text { ness } \\
\text { of } \\
\text { back 。 }\end{array}$} & \multirow{2}{*}{$\begin{array}{c}\text { Density } \\
\text { of pile, } \\
D= \\
2 R P B\end{array}$} & \multirow{2}{*}{$\begin{array}{c}\text { (Density } \\
\text { of pile) } \times \\
\text { (Pile } \\
\text { height) } \\
D H\end{array}$} & \multirow{2}{*}{$\begin{array}{c}\text { (Density } \\
\text { of pile) } \\
\text { (Pile } \\
\text { height) } \\
D^{2} H\end{array}$} & \multicolumn{3}{|c|}{$S$ index } \\
\hline & & & & & & & & & & & & $\underset{\text { machine }}{\text { NBS }}$ & $\begin{array}{c}\text { Shawmut } \\
\text { machine }\end{array}$ & $\begin{array}{c}\text { Service } \\
\text { tests }\end{array}$ \\
\hline Wilton-- & $\begin{array}{r}7.9 \\
8.0 \\
9.9 \\
9.9 \\
11.9 \\
11.8\end{array}$ & $\begin{array}{l}9.4 \\
9.4 \\
9.4 \\
9.3 \\
9.4 \\
9.3\end{array}$ & $\begin{array}{r}\text { Grains } \\
0.097 \\
.097 \\
.088 \\
.102 \\
.093 \\
.096\end{array}$ & $\begin{array}{r}\text { Inches } \\
0.46 \\
.51 \\
.46 \\
.50 \\
.47 \\
.51\end{array}$ & $\begin{array}{r}\text { Grains } \\
0.211 \\
.190 \\
.191 \\
.204 \\
.198 \\
.188\end{array}$ & $\begin{array}{r}\text { Inches } \\
0.17 \\
.19 \\
.17 \\
.19 \\
.17 \\
.19\end{array}$ & $\begin{array}{r}\text { Inches } \\
0.162 \\
.174 \\
.169 \\
.192 \\
.187 \\
.202\end{array}$ & $\begin{array}{c}\text { Inches } \\
0.108 \\
.108 \\
.108 \\
.108 \\
.108 \\
.108\end{array}$ & $\begin{array}{r}\text { Grains/ } \\
\text { in. } \\
31.3 \\
28.6 \\
35.5 \\
37.6 \\
44.3 \\
41.3\end{array}$ & $\begin{array}{r}\text { Grains/ } \\
\text { in. }{ }^{2} \\
5.3 \\
5.4 \\
6.0 \\
7.2 \\
7.5 \\
7.8\end{array}$ & $\begin{array}{c}\text { Grains }{ }^{2 /} \\
\text { in. }^{5} \\
167 \\
155 \\
214 \\
269 \\
334 \\
324\end{array}$ & $\begin{array}{c}1,000 \text { rev- } \\
\text { olutions } \\
34 \\
43 \\
56 \\
62 \\
87 \\
84\end{array}$ & $\begin{array}{r}\text { Hours } \\
7.6 \\
7.3 \\
9.6 \\
8.9 \\
12.0 \\
11.3\end{array}$ & Weeks \\
\hline $\begin{array}{l}1 \\
2 \\
3 \\
4 \\
4 \\
5 \\
6 \\
6\end{array}$ & $\begin{array}{r}8.0 \\
10.0 \\
11.8 \\
10.0 \\
9.9 \\
9.9\end{array}$ & $\begin{array}{l}9.3 \\
9.3 \\
9.3 \\
9.3 \\
9.3 \\
9.3\end{array}$ & $\begin{array}{l}.091 \\
.094 \\
.089 \\
.066 \\
.092 \\
.108\end{array}$ & $\begin{array}{l}.48 \\
.46 \\
.45 \\
.36 \\
.52 \\
.57\end{array}$ & $\begin{array}{l}.189 \\
.204 \\
.198 \\
.183 \\
.177 \\
.189\end{array}$ & $\begin{array}{l}.17 \\
.19 \\
.17 \\
.16 \\
.20 \\
.23\end{array}$ & $\begin{array}{l}.170 \\
.172 \\
.178 \\
.150 \\
.203 \\
.225\end{array}$ & $\begin{array}{l}.105 \\
.107 \\
.110 \\
.105 \\
.107 \\
.107\end{array}$ & $\begin{array}{l}28.1 \\
37.9 \\
43.5 \\
34.0 \\
32.6 \\
34.8\end{array}$ & $\begin{array}{l}4.8 \\
7.2 \\
7.4 \\
5.4 \\
6.5 \\
8.0\end{array}$ & $\begin{array}{l}134 \\
273 \\
322 \\
185 \\
213 \\
278\end{array}$ & $\begin{array}{l}39 \\
78 \\
99 \\
36 \\
71 \\
74\end{array}$ & $\begin{array}{r}10.2 \\
12.0 \\
14.8 \\
9.8 \\
12.5 \\
12.8\end{array}$ & $\begin{array}{l}205 \\
308 \\
370 \\
171 \\
368 \\
398\end{array}$ \\
\hline${ }^{*}$ Chenille_. & $\begin{array}{l}5.1 \\
5.2 \\
5.1 \\
5.9 \\
6.0 \\
6.0 \\
6.8 \\
6.9 \\
6.8\end{array}$ & $\begin{array}{l}10.3 \\
10.5 \\
10.0 \\
12.0 \\
12.2 \\
12.1 \\
14.2 \\
13.6 \\
14.0\end{array}$ & $\begin{array}{l}.123 \\
.148 \\
.191 \\
.126 \\
.148 \\
.199 \\
.121 \\
.155 \\
.216\end{array}$ & $\begin{array}{l}.58 \\
.68 \\
.88 \\
.58 \\
.68 \\
.90 \\
.56 \\
.72 \\
.98\end{array}$ & $\begin{array}{l}.212 \\
.218 \\
.217 \\
.217 \\
.218 \\
.221 \\
.216 \\
.215 \\
.220\end{array}$ & $\begin{array}{l}.26 \\
.29 \\
.41 \\
.24 \\
.31 \\
.40 \\
.26 \\
.33 \\
.46\end{array}$ & $\begin{array}{l}.190 \\
.230 \\
.275 \\
.223 \\
.275 \\
.370 \\
.238 \\
.310 \\
.424\end{array}$ & $\begin{array}{l}.199 \\
.199 \\
.199 \\
.208 \\
.208 \\
.208 \\
.217 \\
.217 \\
.217\end{array}$ & $\begin{array}{l}22.3 \\
23.8 \\
22.1 \\
30.7 \\
31.9 \\
32.1 \\
41.7 \\
40.4 \\
41.9\end{array}$ & $\begin{array}{r}5.8 \\
7.0 \\
9.1 \\
7.4 \\
9.9 \\
12.8 \\
10.9 \\
13.3 \\
19.3\end{array}$ & $\begin{array}{l}129 \\
164 \\
201 \\
226 \\
315 \\
412 \\
452 \\
538 \\
808\end{array}$ & $\begin{array}{r}4.7 \\
5.6 \\
6.0 \\
9.4 \\
11.7 \\
12.1 \\
19.7 \\
18.8 \\
22.5\end{array}$ & $\begin{array}{r}.7 \\
1.0 \\
1.3 \\
1.1 \\
1.5 \\
1.9 \\
2.1 \\
2.1 \\
3.0\end{array}$ & - \\
\hline Axminster & $\begin{array}{l}4.6 \\
4.6 \\
5.4 \\
5.4 \\
7.3 \\
7.3 \\
9.1 \\
9.0\end{array}$ & $\begin{array}{l}\text { 7. } 0 \\
\text { 7. } \\
\text { 7. } 0 \\
\text { 7. } 0 \\
\text { 7. } 0 \\
\text { 7. } 0 \\
\text { 7. } 0 \\
\text { 7. }\end{array}$ & $\begin{array}{l}.140 \\
.187 \\
.137 \\
.192 \\
.139 \\
.186 \\
.138 \\
.195\end{array}$ & $\begin{array}{l}.62 \\
.84 \\
.64 \\
.86 \\
.64 \\
.85 \\
.62 \\
.85\end{array}$ & $\begin{array}{l}.226 \\
.223 \\
.214 \\
.223 \\
.217 \\
.219 \\
.223 \\
.229\end{array}$ & $\begin{array}{l}.20 \\
.28 \\
.19 \\
.28 \\
.20 \\
.28 \\
.22 \\
.31\end{array}$ & $\begin{array}{l}.116 \\
.150 \\
.119 \\
.187 \\
.174 \\
.262 \\
.188 \\
.305\end{array}$ & $\begin{array}{l}.133 \\
.133 \\
.146 \\
.146 \\
.140 \\
.140 \\
.140 \\
.140\end{array}$ & $\begin{array}{l}14.5 \\
14.6 \\
16.2 \\
16.9 \\
22.2 \\
22.4 \\
28.3 \\
28.9\end{array}$ & $\begin{array}{l}2.9 \\
4.1 \\
3.8 \\
4.7 \\
4.4 \\
6.3 \\
6.2 \\
9.0\end{array}$ & $\begin{array}{r}42 \\
60 \\
61 \\
80 \\
99 \\
140 \\
176 \\
259\end{array}$ & $\begin{array}{r}6.6 \\
6.9 \\
8.4 \\
12.3 \\
21.3 \\
24.8 \\
30.3 \\
40.3\end{array}$ & $\begin{array}{l}2.8 \\
1.4 \\
1.5 \\
2.0 \\
3.9 \\
2.8 \\
4.0 \\
4.2\end{array}$ & 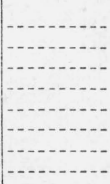 \\
\hline $\begin{array}{l}14 \\
13 \\
12 \\
11 \\
8 \\
10 \\
9 \\
7\end{array}$ & $\begin{array}{l}4.2 \\
4.4 \\
4.7 \\
4.8 \\
6.2 \\
8.0 \\
6.1 \\
6.1\end{array}$ & $\begin{array}{l}\text { 7. } 0 \\
\text { 7. } \\
\text { 7. } 0 \\
7.0 \\
\text { 7. } 0 \\
\text { 7. } 0 \\
\text { 7. } 0 \\
\text { 7. } 0\end{array}$ & $\begin{array}{l}.171 \\
.173 \\
.175 \\
.175 \\
.167 \\
.196 \\
.133 \\
.210\end{array}$ & $\begin{array}{r}.86 \\
.87 \\
.84 \\
.85 \\
.86 \\
.87 \\
.65 \\
1.04\end{array}$ & $\begin{array}{l}.199 \\
.199 \\
.208 \\
.206 \\
.194 \\
.225 \\
.205 \\
.202\end{array}$ & $\begin{array}{l}.33 \\
.33 \\
.33 \\
.33 \\
.33 \\
.33 \\
.23 \\
.42\end{array}$ & $\begin{array}{l}.151 \\
.139 \\
.146 \\
.164 \\
.212 \\
.295 \\
.180 \\
.255\end{array}$ & $\begin{array}{l}.125 \\
.125 \\
.130 \\
.130 \\
.135 \\
.135 \\
.135 \\
.135\end{array}$ & $\begin{array}{l}11.6 \\
12.4 \\
13.5 \\
13.8 \\
16.8 \\
25.2 \\
17.5 \\
17.3\end{array}$ & $\begin{array}{l}3.8 \\
4.1 \\
4.5 \\
4.6 \\
5.5 \\
8.3 \\
4.0 \\
7.3\end{array}$ & $\begin{array}{r}44 \\
51 \\
60 \\
63 \\
93 \\
210 \\
70 \\
126\end{array}$ & $\begin{array}{r}5.6 \\
8.0 \\
8.8 \\
11.6 \\
22.0 \\
44.2 \\
21.8 \\
23.2\end{array}$ & \begin{tabular}{r|r}
1.5 \\
3.5 \\
2.8 \\
3.8 \\
7.2 \\
10.0 \\
6.4 \\
7.9
\end{tabular} & $\begin{array}{r}65 \\
70 \\
80 \\
102 \\
152 \\
260 \\
114 \\
220\end{array}$ \\
\hline
\end{tabular}




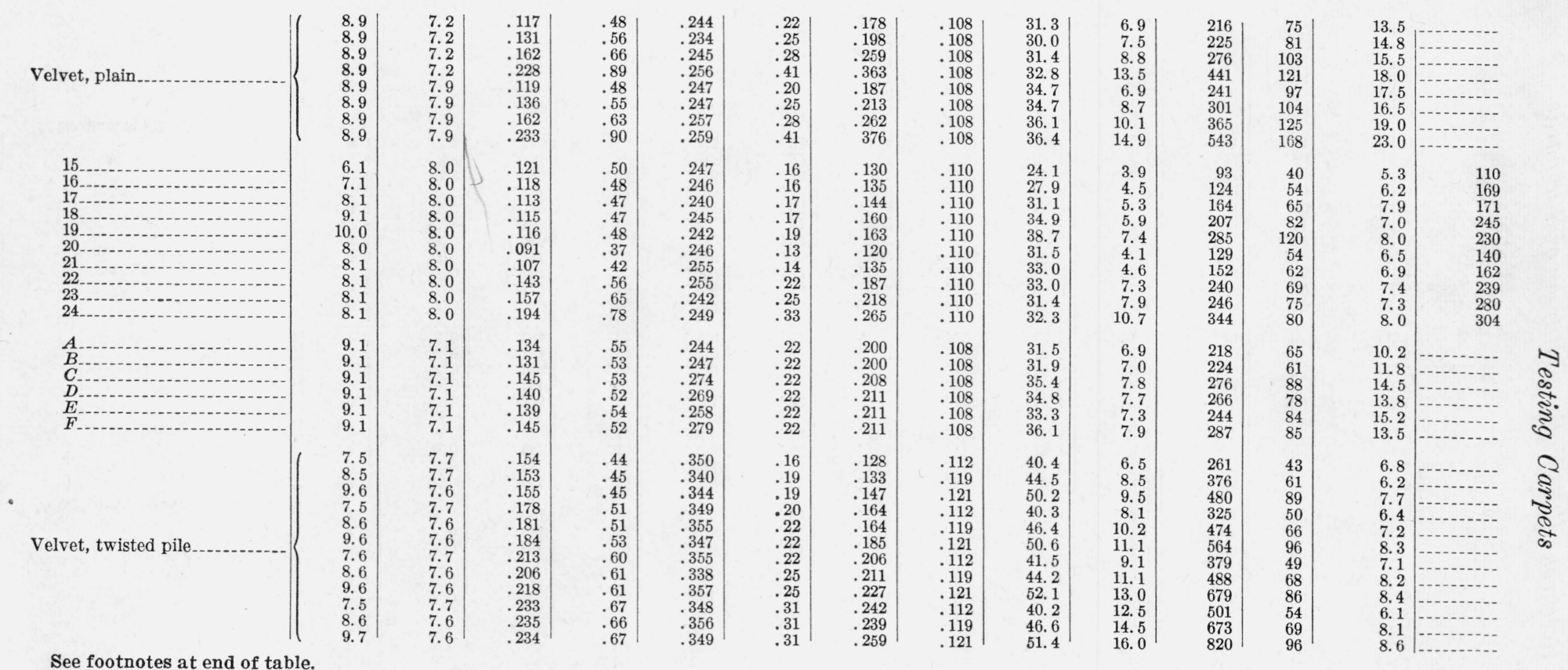


TABLE 2.-Analysis of carpets and test results-Continued

\begin{tabular}{|c|c|c|c|c|c|c|c|c|c|c|c|c|c|c|}
\hline \multirow{2}{*}{ Weave $\mathrm{s}$} & \multirow{2}{*}{$\begin{array}{l}\text { Rows } \\
\text { per } \\
\text { inch, } \\
R\end{array}$} & \multirow{2}{*}{$\begin{array}{l}\text { Pitch } \\
\text { per } \\
\text { inch, } \\
P\end{array}$} & \multirow{2}{*}{$\begin{array}{c}\text { Weight } \\
\text { per } \\
\text { tuft, } \\
\text { W }\end{array}$} & \multirow{2}{*}{$\begin{array}{l}\text { Length } \\
\text { of } \\
\text { tuft, } \\
L\end{array}$} & \multirow{2}{*}{$\begin{array}{l}\text { Weight } \\
\text { of tuft } \\
\text { per inch } \\
\text { length } \\
B=W / L\end{array}$} & \multirow{2}{*}{$\begin{array}{c}\text { Height } \\
\text { of } \\
\text { pile, b } \\
H\end{array}$} & \multirow{2}{*}{$\begin{array}{l}\text { Initial } \\
\text { thick- } \\
\text { ness } \\
\text { of } \\
\text { pile c }\end{array}$} & \multirow{2}{*}{$\begin{array}{l}\text { Initial } \\
\text { thick- } \\
\text { ness } \\
\text { of } \\
\text { back c }\end{array}$} & \multirow{2}{*}{$\begin{array}{c}\text { Density } \\
\text { of pile, } \\
D= \\
2 R P B\end{array}$} & \multirow{2}{*}{$\begin{array}{c}\text { (Density } \\
\text { of pile) } \\
\text { (Pile } \\
\text { height) } \\
D H\end{array}$} & \multirow{2}{*}{$\begin{array}{c}\text { (Density } \\
\text { of pile) })^{2} \times \\
\text { (Pile } \\
\text { height) } \\
D^{2} H\end{array}$} & \multicolumn{3}{|c|}{$S$ index } \\
\hline & & & & & & & & & & & & $\begin{array}{c}\mathrm{NBS} \\
\text { machine }\end{array}$ & $\begin{array}{l}\text { Shawmut } \\
\text { machine }\end{array}$ & $\begin{array}{c}\text { Service } \\
\text { tests }\end{array}$ \\
\hline \multirow{5}{*}{ Velvet, printed . . } & $\begin{array}{l}7.0 \\
7.0 \\
7.0 \\
8.9 \\
8.9 \\
8.9\end{array}$ & $\begin{array}{l}8.0 \\
8.0 \\
8.0 \\
7.9 \\
8.0 \\
7.9\end{array}$ & $\begin{array}{c}\text { Grains } \\
0.199 \\
.287 \\
.358 \\
.194 \\
.298 \\
.324\end{array}$ & $\begin{array}{r}\text { Inches } \\
0.58 \\
.82 \\
.94 \\
.58 \\
.85 \\
.95\end{array}$ & $\begin{array}{c}\text { Grains } \\
0.343 \\
.350 \\
.381 \\
.335 \\
.351 \\
.341\end{array}$ & $\begin{array}{r}\text { Inches } \\
0.25 \\
.34 \\
.39 \\
.25 \\
.34 \\
.41\end{array}$ & $\begin{array}{c}\text { Inches } \\
0.223 \\
.339 \\
.390 \\
.230 \\
.358 \\
.416\end{array}$ & $\begin{array}{c}\text { Inches } \\
0.128 \\
.128 \\
.128 \\
.133 \\
.133 \\
.133\end{array}$ & $\begin{array}{c}\text { Grains/ } \\
\text { in. } 3 \\
38.4 \\
39.2 \\
42.7 \\
47.1 \\
50.0 \\
47.9\end{array}$ & \begin{tabular}{|r|}
$\begin{array}{c}\text { Grains/ } \\
\text { in. }\end{array}$ \\
9.6 \\
13.3 \\
16.7 \\
11.8 \\
17.0 \\
19.6
\end{tabular} & \begin{tabular}{|r|} 
Grains $^{2 /}$ \\
in. $^{5}$ \\
369 \\
522 \\
711 \\
554 \\
850 \\
941
\end{tabular} & \begin{tabular}{|c}
1,000 rev- \\
olutions \\
71 \\
92 \\
105 \\
149 \\
172 \\
171
\end{tabular} & $\begin{array}{c}\text { Hours } \\
\text { e } 3.0 \\
\text { e 2.2 } \\
e 3.3 \\
\text { e } 3.3 \\
\text { e } 5.6 \\
\text { e } 7.4\end{array}$ & (1) \\
\hline & $\begin{array}{l}9.0 \\
9.1 \\
9.0\end{array}$ & $\begin{array}{l}8.0 \\
8.0 \\
8.0\end{array}$ & $\begin{array}{l}.151 \\
.153 \\
.150\end{array}$ & $\begin{array}{l}.42 \\
.42 \\
.40\end{array}$ & $\begin{array}{l}.360 \\
.364 \\
.375\end{array}$ & $\begin{array}{l}.19 \\
.19 \\
.19\end{array}$ & $\begin{array}{l}.175 \\
.171 \\
.176\end{array}$ & $\begin{array}{l}.110 \\
.110 \\
.110\end{array}$ & $\begin{array}{l}51.8 \\
53.0 \\
54.0\end{array}$ & $\begin{array}{r}9.9 \\
10.1 \\
10.3\end{array}$ & $\begin{array}{l}510 \\
534 \\
554\end{array}$ & $\begin{array}{l}(t) \\
(t) \\
(t)\end{array}$ & $\begin{array}{l}18 \\
86 \\
15.8\end{array}$ & \\
\hline & $\begin{array}{l}9.0 \\
9.0 \\
9.0 \\
9.0\end{array}$ & $\begin{array}{l}8.0 \\
8.0 \\
8.0 \\
8.0\end{array}$ & $\begin{array}{l}.134 \\
.141 \\
.137 \\
.139\end{array}$ & $\begin{array}{l}.45 \\
.45 \\
.45 \\
.45\end{array}$ & $\begin{array}{l}.298 \\
.313 \\
.304 \\
.309\end{array}$ & $\begin{array}{l}.16 \\
.16 \\
.16 \\
.16\end{array}$ & $\begin{array}{l}.170 \\
.167 \\
.168 \\
.170\end{array}$ & $\begin{array}{l}.115 \\
.115 \\
.115 \\
.115\end{array}$ & $\begin{array}{l}42.9 \\
45.1 \\
43.8 \\
44.5\end{array}$ & $\begin{array}{l}6.9 \\
7.2 \\
7.0 \\
7.1\end{array}$ & $\begin{array}{l}295 \\
325 \\
307 \\
317\end{array}$ & $\begin{array}{l}\text { (f) } \\
\text { (i) } \\
\text { (f) } \\
\text { (i) }\end{array}$ & $\begin{array}{l}14.0 \\
14.3 \\
16.0 \\
17.0\end{array}$ & \\
\hline & $\begin{array}{l}9.0 \\
9.0 \\
8.9 \\
9.0 \\
9.0\end{array}$ & $\begin{array}{l}7.9 \\
8.0 \\
8.0 \\
8.0 \\
8.0\end{array}$ & $\begin{array}{l}.206 \\
.191 \\
.196 \\
.188 \\
.188\end{array}$ & $\begin{array}{l}.57 \\
.57 \\
.56 \\
.57 \\
.57\end{array}$ & $\begin{array}{l}.361 \\
.335 \\
.350 \\
.330 \\
.330\end{array}$ & $\begin{array}{l}.23 \\
.23 \\
.23 \\
.23 \\
.23\end{array}$ & - & $\begin{array}{l}.125 \\
.125 \\
.125 \\
.125 \\
.125\end{array}$ & $\begin{array}{l}51.4 \\
48.2 \\
49.8 \\
47.5 \\
47.5\end{array}$ & $\begin{array}{l}11.8 \\
11.1 \\
11.5 \\
10.9 \\
10.9\end{array}$ & \begin{tabular}{l|}
608 \\
534 \\
570 \\
519 \\
519
\end{tabular} & $\begin{array}{l}\text { (1) } \\
\text { (1) } \\
\text { (1) } \\
\text { (1) } \\
(1)\end{array}$ & \begin{tabular}{l|}
11.1 \\
15.3 \\
13.3 \\
e 7.6 \\
07.1
\end{tabular} & \\
\hline & $\begin{array}{r}9.3 \\
8.0 \\
9.3 \\
9.3 \\
9.1 \\
\text { g } 7.8 \\
\mathrm{~h} 4.7 \\
i .7 \\
12.0 \\
8.4 \\
9.1 \\
9.1\end{array}$ & $\begin{array}{l}7.9 \\
8.0 \\
7.9 \\
7.9 \\
7.9 \\
7.8 \\
7.0 \\
6.7 \\
9.3 \\
7.8 \\
7.3\end{array}$ & $\begin{array}{r}.213 \\
.112 \\
.197 \\
.221 \\
.243 \\
.163 \\
.720 \\
.082 \\
.137 \\
.134\end{array}$ & $\begin{array}{r}.65 \\
.49 \\
.65 \\
.69 \\
.71 \\
1.09 \\
.70 \\
1.21 \\
.44 \\
.56 \\
.55\end{array}$ & $\begin{array}{l}.328 \\
.229 \\
.303 \\
.320 \\
.342 \\
.150 \\
.241 \\
.595 \\
.186 \\
.245 \\
.245\end{array}$ & $\begin{array}{l}.25 \\
.16 \\
.27 \\
.25 \\
.27 \\
.25 \\
.47 \\
.19 \\
.22 \\
.22\end{array}$ & $\begin{array}{l}.268 \\
.165 \\
.261 \\
.228 \\
.257 \\
.212 \\
.164 \\
.400 \\
.161 \\
.194 \\
.184\end{array}$ & $\begin{array}{l}.109 \\
.107 \\
.102 \\
.133 \\
.125 \\
.113 \\
.133 \\
.115 \\
.110 \\
.132 \\
.111\end{array}$ & $\begin{array}{l}48.4 \\
29.3 \\
44.5 \\
47.0 \\
49.2 \\
18.2 \\
15.9 \\
55.8 \\
41.5 \\
32.1 \\
32.4\end{array}$ & $\begin{array}{r}12.1 \\
4.7 \\
12.0 \\
11.8 \\
13.3 \\
7.5 \\
4.0 \\
26.2 \\
7.9 \\
7.1 \\
7.1\end{array}$ & $\begin{array}{r}586 \\
137 \\
534 \\
552 \\
654 \\
136 \\
64 \\
1463 \\
327 \\
227 \\
231\end{array}$ & $\begin{array}{r}137 \\
40 \\
96 \\
260 \\
262 \\
171 \\
20 \\
73 \\
68 \\
.63 \\
81\end{array}$ & $\begin{array}{r}17.0 \\
12.8 \\
17.4 \\
8.0 \\
12.3 \\
6.2 \\
20.0 \\
10.0 \\
\mathrm{e} 6.0 \\
12.5 \\
14.0\end{array}$ & \\
\hline & & & & & & & $\begin{array}{r}.440 \\
.250\end{array}$ & $\begin{array}{r}.133 \\
.142\end{array}$ & & & & $\begin{array}{l}124 \\
{ }_{318}\end{array}$ & $\begin{array}{r}20.0 \\
17.0\end{array}$ & \\
\hline & & & & & 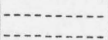 & 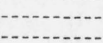 & $\begin{array}{r}.237 \\
.274\end{array}$ & $\begin{array}{l}.105 \\
.140\end{array}$ & & & & $\begin{array}{r}154 \\
47\end{array}$ & $\begin{array}{l}11.0 .0 \\
10.0\end{array}$ & 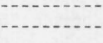 \\
\hline & & & & & & & $\begin{array}{l}.274 \\
.286\end{array}$ & $\begin{array}{l}.115 \\
.115\end{array}$ & & & & $\begin{array}{r}91 \\
90 \\
140\end{array}$ & $\begin{array}{r}8.0 \\
83.0\end{array}$ & \\
\hline
\end{tabular}




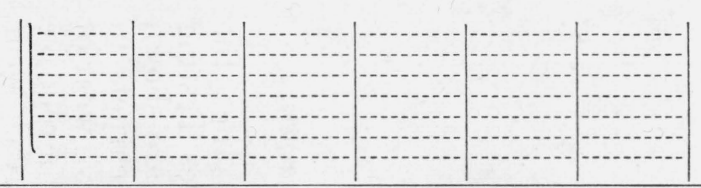

.257
.300
.562
.499
.318
.260
.308
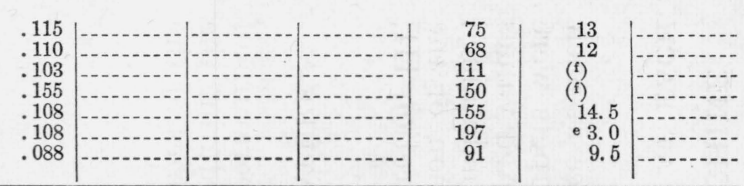

a Numerals indicate the number designation of the service-wear tests. Capital letters indicate a series of carpets in which 20 percent of various wastes were added to the base blend of the pile used in carpet $A$.

- Measured with a gage having a circular foot $1 \mathrm{in}$. in diameter and exerting a pressure of $0.75 \mathrm{lb} / \mathrm{in} .^{2}$

d Two-shot construction.

excessive heating and shimmying were produced in the test with the Shawmut machine.

1 Not tested.

h Jute pile. 50 percent of rayon and 50 percent of wool rile.
i Twisted pile.

*The wool forming the pile of the chenille carpets showed serious degradation according to the alkali-solubility test. This degradation of wool and the low wear indices which were obtained is not typical of commercial production of chenille or other carpets. The results for these chenille carpets are included in this paper because they do furnish an excellent example of the definite relationship which exists between the wear test results and the chemical degradation of the wool forming the pile of a carpet. 


\section{STANDARDIZATION OF TEST ON NBS MACHINE}

\section{EFFECT OF BRAKING TORQUE, DOWNWARD FORCE AT EACH PULLEY, AND SPEED OF TURNTABLE}

The effects of varying the braking torque, downward force at each pulley, and the speed of the turntable on the wear of carpets were reported in Research Paper RP315. ${ }^{7}$ It was found that fixed values must be set for these quantities, and these values must be maintained constant during each test in order to effect standardization of the test. The values for these quantities which are given in section III, $3 \mathrm{a}$, are based upon these tests.

\section{EFFECT OF RELATIVE HUMIDITY AND TEMPERATURE}

Tests, to show the effect of relative humidity and temperature of the air with which the carpet specimens are in equilibrium during the wear test on the NBS machine were made in the laboratories of the

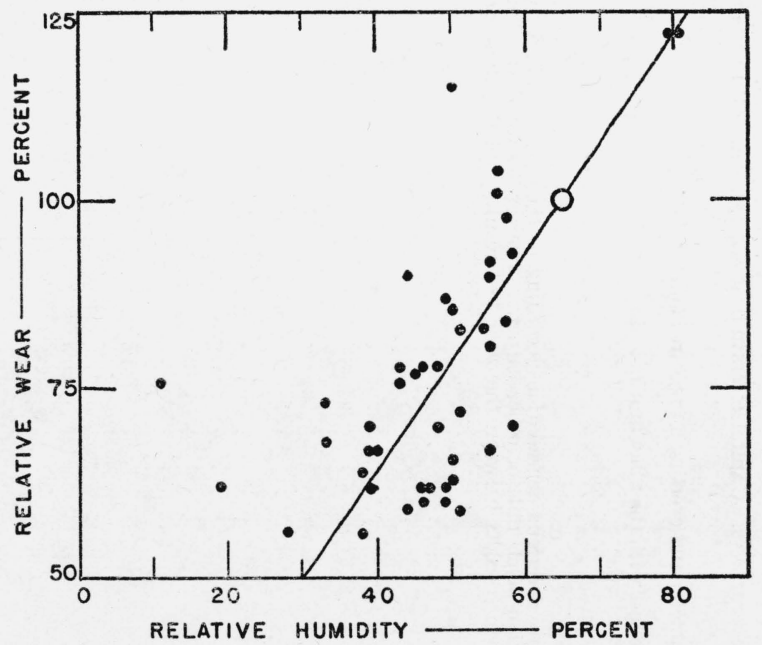

FIGURE 13.-Effect of relative humidity on the relative wear of carpets.

Bigelow Sanford Carpet Co., the Mohawk Carpet Mills, Inc., the Alexander Smith \& Sons Carpet Co., and the United States Testing Co. The results of the tests at different relative humidities are shown graphically in figure 13, where the relative wear of the carpets in percent is plotted against the relative humidity. The relative wear of each carpet tested is taken to be 100 percent when tested at a relative humidity of 65 percent. The results show that the relative wear increases significantly with the relative humidity. A 10-percent change in relative humidity from 65 percent corresponds to a 15percent change in the relative wear of the carpets. The data regarding the effect of the temperature of the air on the relative wear of carpets are rather meager. The relative wear of a carpet appears to decrease as the temperature of the air is increased. It is thus important that the wear tests of carpets be made in an atmosphere of controlled temperature and relative humidity.

\footnotetext{
${ }^{7}$ See footnote 1 on p. 334 .
} 


\section{EFFECT OF HEIGHT OF NOZZLE AND SUCTION AT THE NOZZLE}

Twelve carpets were tested to show the effects of the height of the nozzle above the pile and of the amount of suction at the nozzle. The wear indices of these carpets increased an average of 3 percent when the height was decreased from $1 / 8$ to $1 / 16$ in. "This increase results from less effective cleaning because of the decrease in the volume of air entering the nozzle as it is lowered from $1 / 8$ to $1 / 16$ in. The wear indices increased an average of 75 percent when the height was increased from $1 / 8$ to $3 / 8$ in. This large increase is attributed to ineffective cleaning at the $3 / 8$-in. height. These results indicate that the optimum height of the nozzle above the pile is between $1 / 8$ and $1 / 16 \mathrm{in}$. The wear indices increased an average of 24 percent when the suction was decreased from 4 to $2 \mathrm{~cm} \mathrm{Hg}$. This increase is attributed to less effective cleaning at the lower suction. It is apparent that the height of the nozzle above the pile and the amount of suction must be maintained at fixed values. Obviously the dimensions of the opening of the nozzle and the position of the suction connection relative to the opening of the nozzle must also be fixed to effect standardization of the test with this machine.

\section{RESULTS}

\section{WEAR INDICES}

The cumulative frequencies, in percentages, of the deviations of the individual wear tests for the $K, S$, and $E$ indices obtained with the NBS and Shawmut machines are given in table 3. The deviation of a test from the average of several tests on the carpet includes the variation between the specimens tested, the experimental errors in singeing the pile from a carpet specimen and in measuring the back thickness, in measuring the total thickness of a carpet before and during a wear test, in obtaining the matted pile thickness by extrapolation of the wear curve, and in operating the wear-testing machines, and small changes in atmospheric conditions. In view of these different errors which enter in the measurement of the wear of a carpet, it seems rather remarkable that the deviation of a single wear test is less than 10 percent in 9 trials out of 10 . On the basis of the frequency distributions of the deviations for the three wear indices, it is concluded that the $S$ index is more reliable than either the $K$ or the $E$ index. This conclusion applies to both the NBS and the Shawmut machines. The larger deviation for the $K$ index probably results because the initial pile thickness, which enters in this index, varies more between specimens because of partial matting of the pile in the handling of the carpet after manufacturing. The initial pile thickness as measured by the Shawmut machine is uncertain, for it is based upon one measurement only and it is also greatly influenced by the mounting of the specimen in the machine. The larger deviation for the $E$ index probably results because of the uncertainty of determining the point of intersection of the pile thickness curve with the abscissa axis. This determination frequently involves some extrapolation of the curve, which becomes nearly paralled to the axis at the end of a test. 
TABLE 3.-Cumulative frequencies, in percentages, of deviations of the individual wear tests for the $K, S$, and $E$ indices obtained with the NBS and Shawmut machines

[The deviation of a test on a carpet is expressed as a percentage of the average of two or more tests on the carpet]

\begin{tabular}{|c|c|c|c|c|c|c|}
\hline \multirow{3}{*}{ Deviation } & \multicolumn{6}{|c|}{ Cumulative frequency } \\
\hline & \multicolumn{3}{|c|}{ NBS wear indices a } & \multicolumn{3}{|c|}{ Shawmut wear indices b } \\
\hline & $K$ & $S$ & $E$ & $K$ & $S$ & $E$ \\
\hline Percent & $\begin{array}{r}\text { Percent } \\
11\end{array}$ & $\underset{10}{\text { Percent }}$ & ${ }_{11}^{\text {Percent }}$ & $\underset{12}{\text { Percent }}$ & $\begin{array}{r}\text { Percent } \\
13\end{array}$ & Percent \\
\hline $\begin{array}{l}1 \\
\\
4\end{array}$ & $\begin{array}{l}25 \\
35 \\
47 \\
59 \\
65\end{array}$ & $\begin{array}{l}27 \\
38 \\
45 \\
53 \\
63\end{array}$ & $\begin{array}{l}19 \\
38 \\
48 \\
61 \\
65\end{array}$ & $\begin{array}{l}20 \\
26 \\
36 \\
42 \\
55\end{array}$ & $\begin{array}{l}23 \\
36 \\
48 \\
61 \\
70\end{array}$ & $\begin{array}{l}23 \\
38 \\
51 \\
62 \\
71\end{array}$ \\
\hline $\begin{array}{l}6 \\
6 \\
\end{array}$ & $\begin{array}{l}75 \\
79 \\
85 \\
89 \\
89\end{array}$ & $\begin{array}{l}77 \\
79 \\
87 \\
88 \\
91\end{array}$ & $\begin{array}{l}75 \\
79 \\
83 \\
83 \\
89\end{array}$ & $\begin{array}{l}58 \\
64 \\
69 \\
75 \\
78\end{array}$ & $\begin{array}{l}77 \\
81 \\
86 \\
90 \\
93\end{array}$ & $\begin{array}{l}76 \\
80 \\
83 \\
86 \\
90\end{array}$ \\
\hline $\begin{array}{l}11 \\
12 \\
13 \\
13\end{array}$ & $\begin{array}{l}93 \\
95 \\
97 \\
97 \\
97\end{array}$ & $\begin{array}{r}94 \\
97 \\
98 \\
100 \\
\end{array}$ & $\begin{array}{l}90 \\
93 \\
94 \\
95 \\
95\end{array}$ & $\begin{array}{l}81 \\
82 \\
86 \\
90 \\
90\end{array}$ & $\begin{array}{l}95 \\
97 \\
98 \\
98 \\
98\end{array}$ & $\begin{array}{l}92 \\
95 \\
96 \\
96 \\
97\end{array}$ \\
\hline 16 & $\begin{array}{l}98 \\
98 \\
98 \\
98 \\
98\end{array}$ & 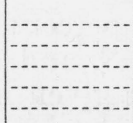 & $\begin{array}{l}95 \\
96 \\
97 \\
97 \\
98\end{array}$ & $\begin{array}{l}90 \\
92 \\
94 \\
95 \\
97\end{array}$ & $\begin{array}{r}99 \\
99 \\
99 \\
99 \\
100\end{array}$ & $\begin{array}{r}97 \\
97 \\
98 \\
99 \\
100\end{array}$ \\
\hline 21 & $\begin{array}{l}98 \\
98 \\
99 \\
99 \\
99\end{array}$ & 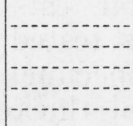 & $\begin{array}{l}98 \\
98 \\
98 \\
98 \\
98\end{array}$ & $\begin{array}{l}97 \\
97 \\
98 \\
98 \\
99\end{array}$ & 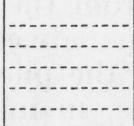 & 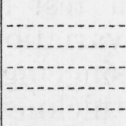 \\
\hline $\begin{array}{l}26 \\
27 \\
28 \\
29\end{array}$ & 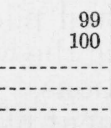 & 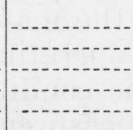 & $\begin{array}{r}98 \\
98 \\
98 \\
99 \\
100\end{array}$ & $\begin{array}{r}99 \\
100 \\
\end{array}$ & 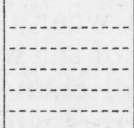 & - \\
\hline
\end{tabular}

a The NBS results are based upon 179 tests on 85 carpets, 2.1 tests per carpet.

b The Shawmut results are based upon 210 tests on 86 carpets, 2.4 tests per carpet.

The relationship between the $S$ index and the $K$ and $E$ indices is shown in figures 14, 15, and 16 for the NBS, Shawmut, and service tests, respectively. A good linear relationship is shown between the indices, although the points are scattered considerably more for the Shawmut machine. The effect of the greater matting of the jute and of the part-rayon-pile carpets is indicated by the NBS machine but not by the Shawmut machine. On the average a test would have to be continued 40 percent beyond the $S$ index to obtain the $E$ index on the NBS machine. The prolongations for the Shawmut and service tests are 100 and 35 percent, respectively. These prolongations of the tests do not seem justified in view of the good linear relationship between the $S$ and $E$ indices. On the other hand, the prolongation of a test to obtain the $S$ index compared with the $K$ index, amounting to 20,50 , and 45 percent of the $S$ index for the NBS, Shawmut, and service tests, respectively, can be justified because of the increase in the precision of the results and also, as shown in the next section, because the $S$ index of the service tests corresponds 
more nearly with the time at which a carpet would be discarded on account of visible wear.

\section{SERVICE TESTS}

Photographs of the middle strip of the carpets after 162 weeks of

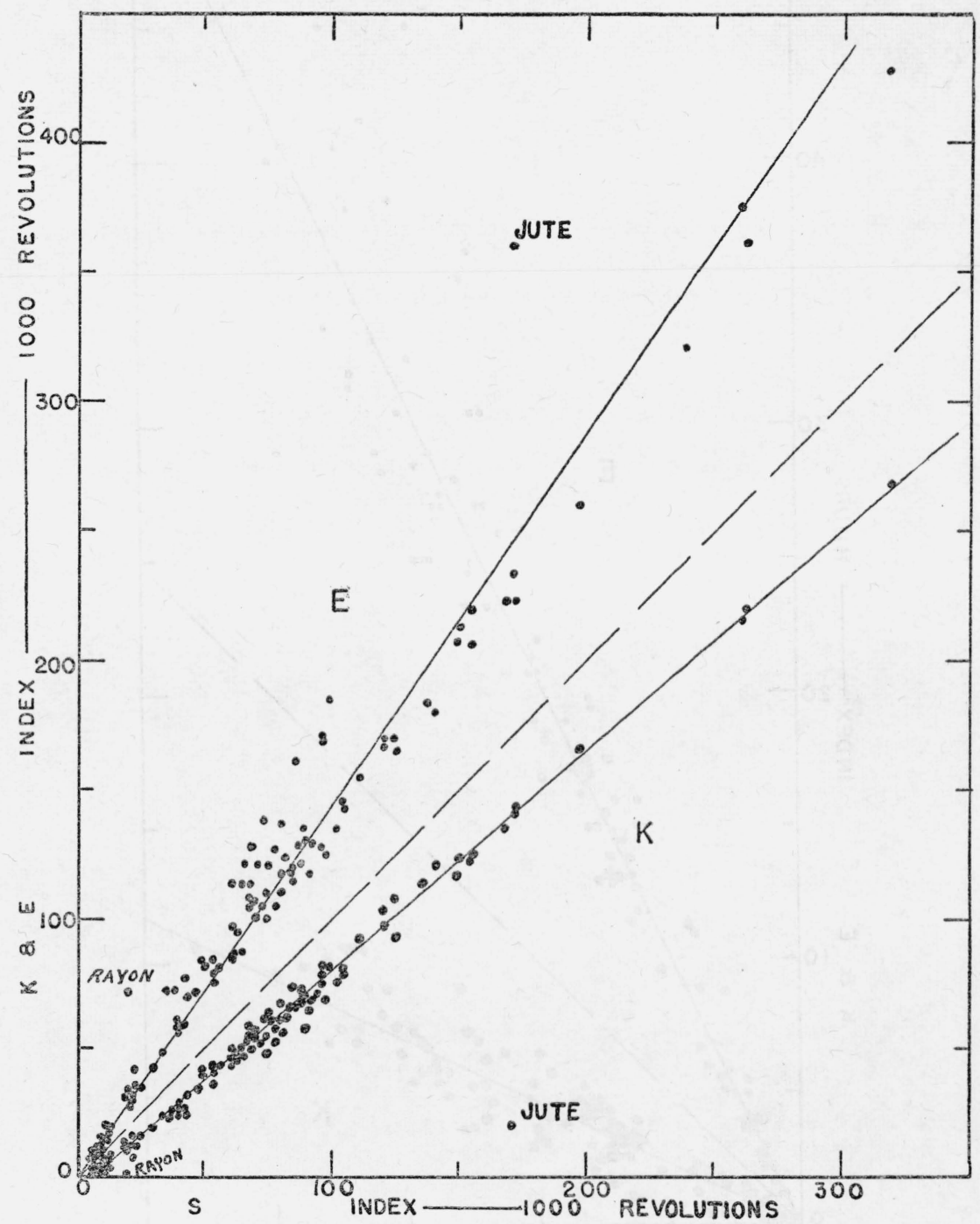

FIGURE 14.-Relationship between $S$ and $K$ and $E$ indices of carpets obtained with the NBS machine.

service wear, followed by washing in a commercial carpet-cleaning plant, are shown in figures 17 to 29 , inclusive. The photographs and the discussion of the measurements of thickness of the carpets are confined to the middle strip because it received more uniform wear. 


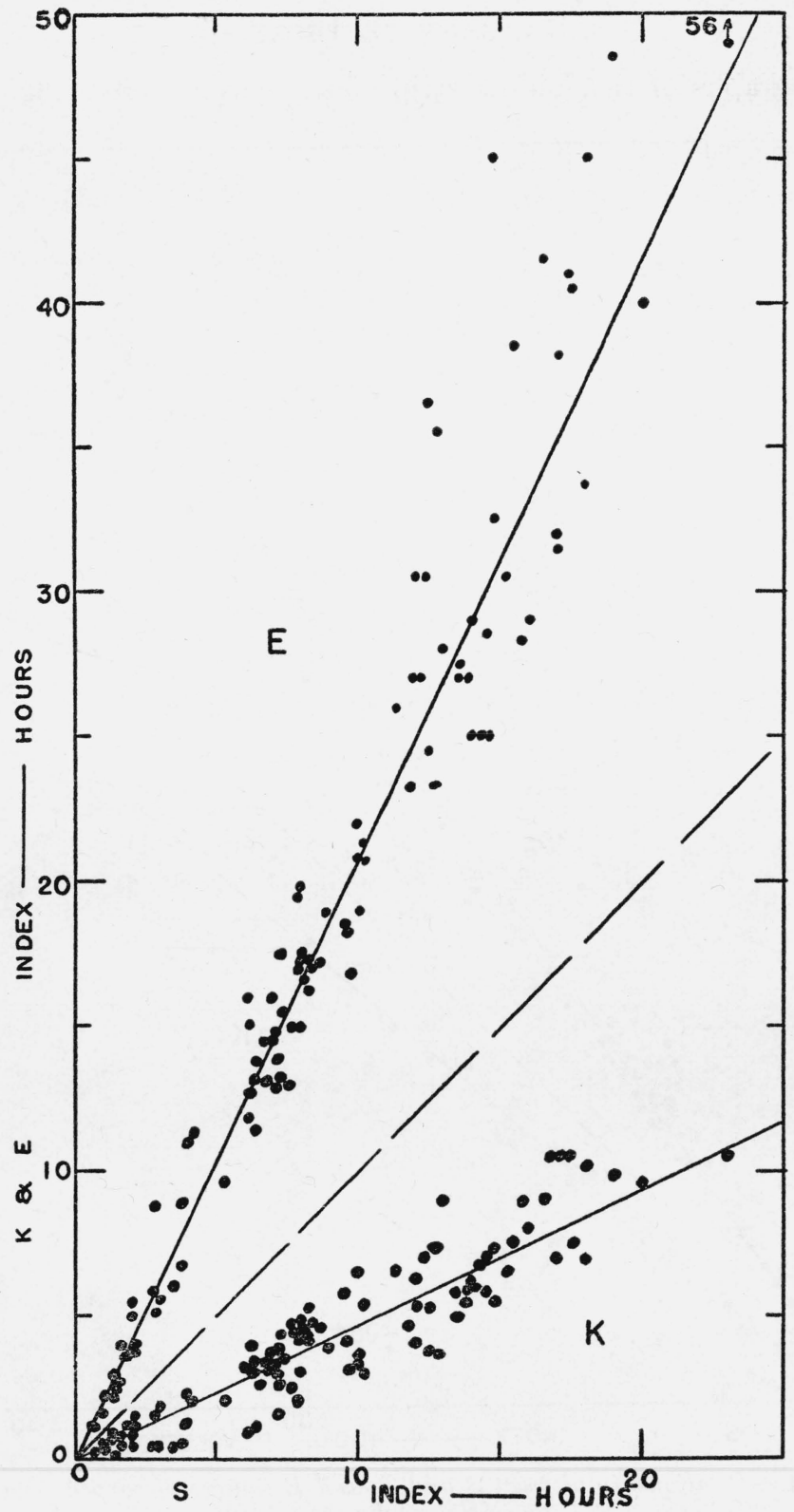

FIGURE 15.-Relationship between $S$ and $K$ and $E$ indices of carpets obtained with the Shawmut machine. 

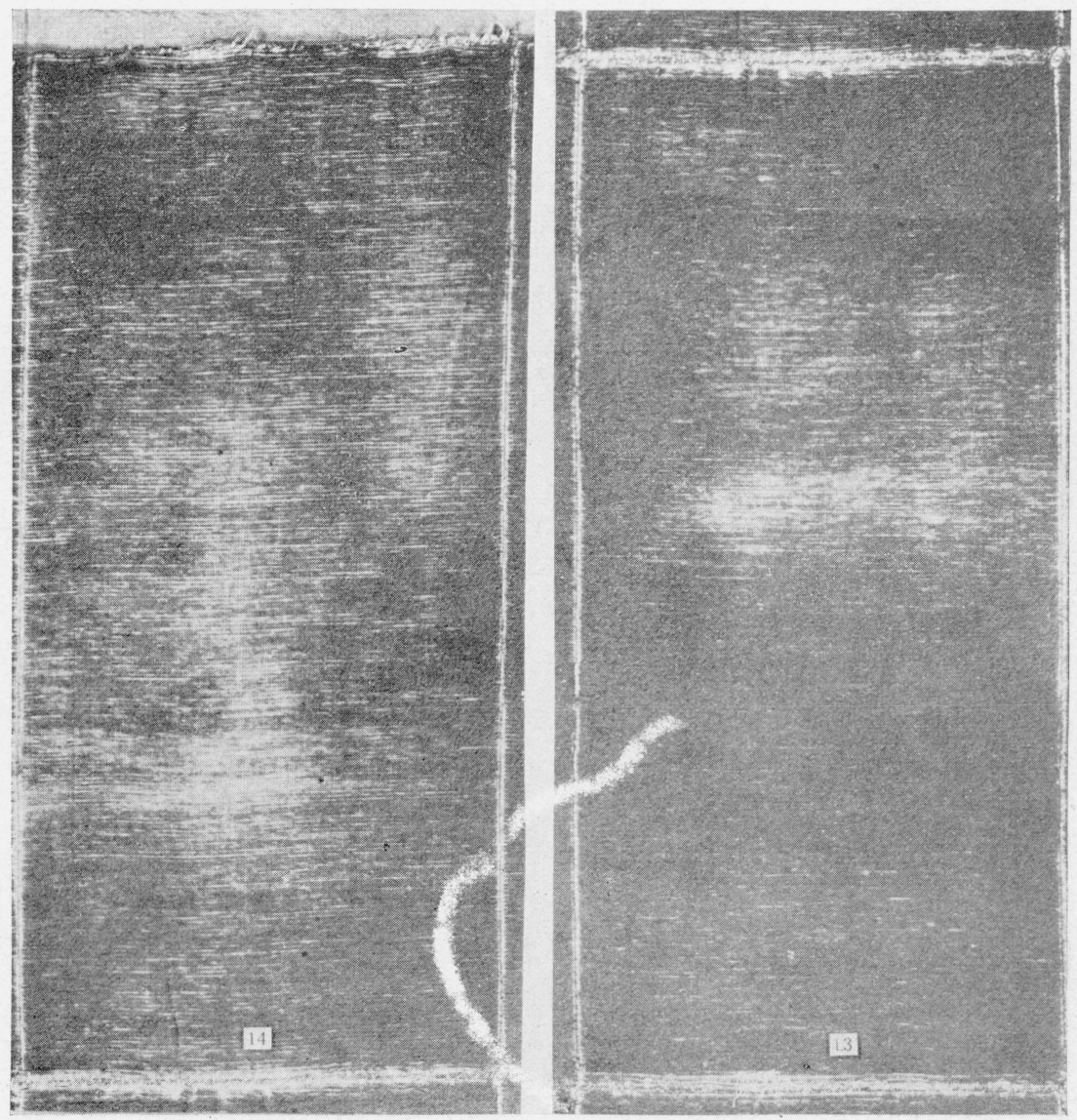

Figure 17.-Carpets 14 and 13 after 162 weeks of service wear; S indices, 65 and 70 weeks, respectively. 


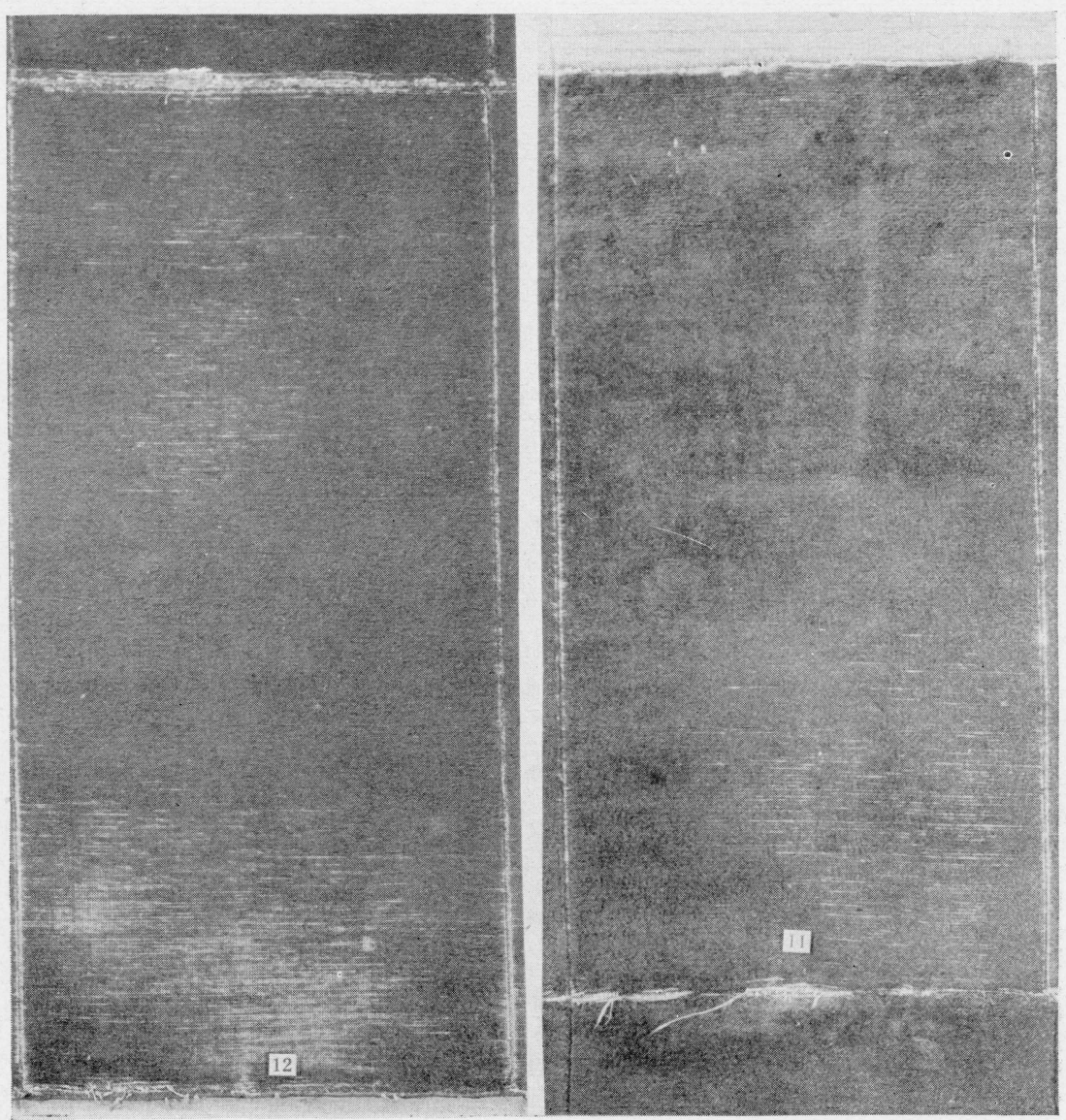

FIGURE 18.-Carpets 12 and 11 after 162 weeks of service wear; $S$ indices, 80 and 102 weeks, respectively. 

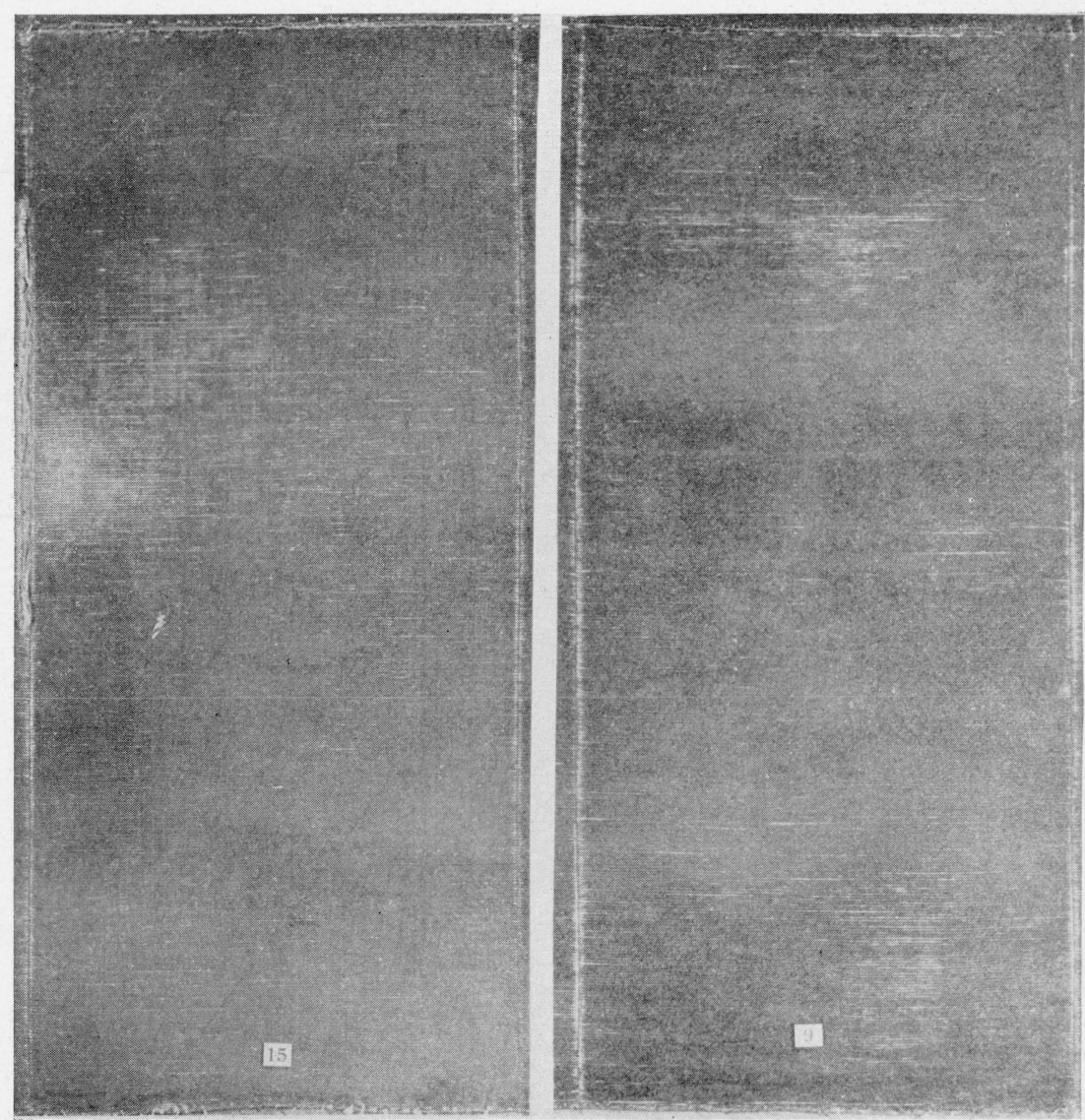

Figure 19.-Carpets 15 and 9 after 162 weeks of service wear; S indices, 110 and 114 weeks, respectively. 

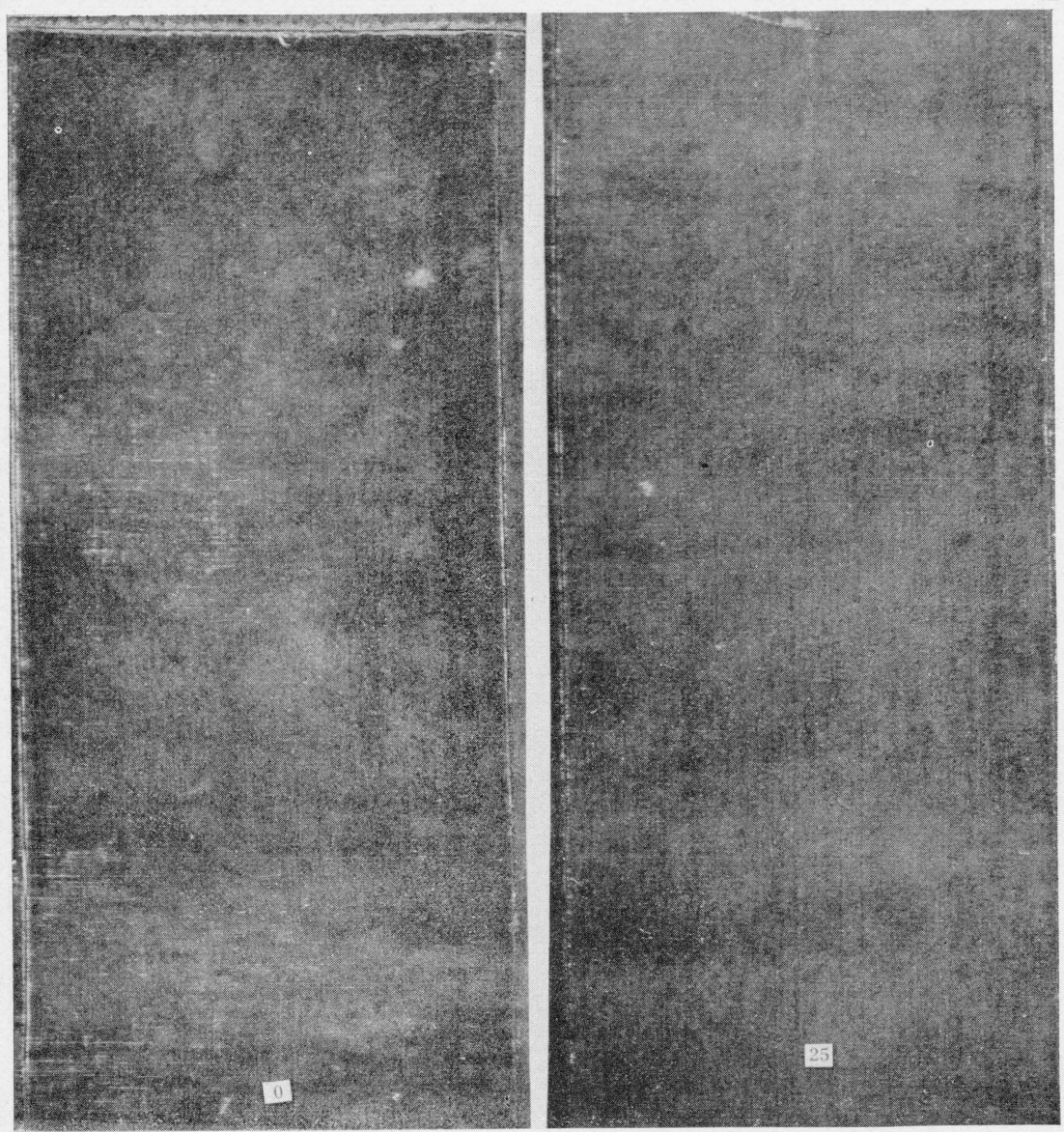

Figure 20.-Carpets 0 and 25 after 162 weeks of service wear; S indices, 138 and 150 weeks, respectively.

Carpet 0 was located at the east end of the corridor and carpet 25 was located at the west end. 

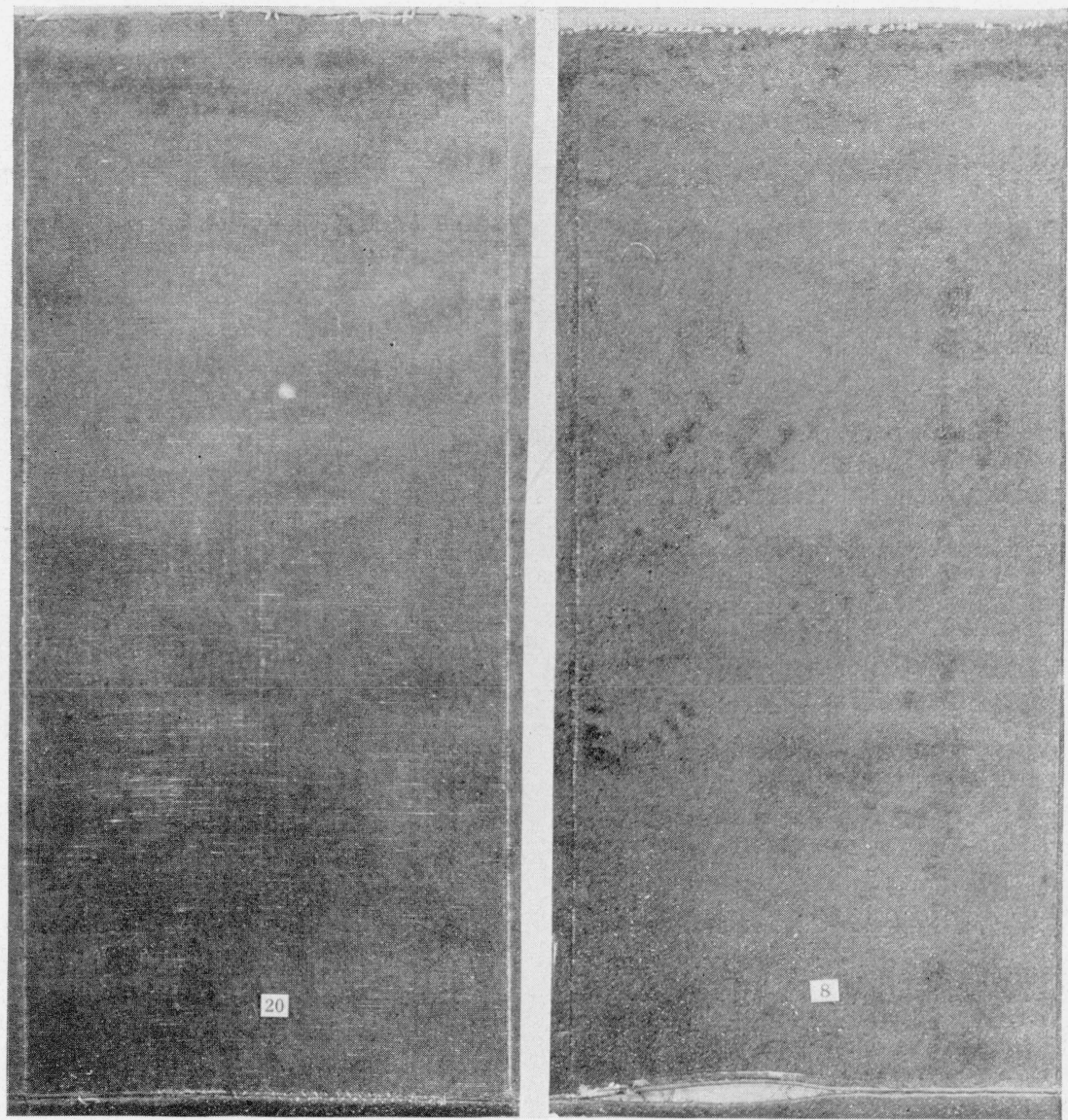

FIGURE 21.-Carpets 20 and 8 after 162 weeks of service wear; S indices, 140 and 152 weeks, respectively. 


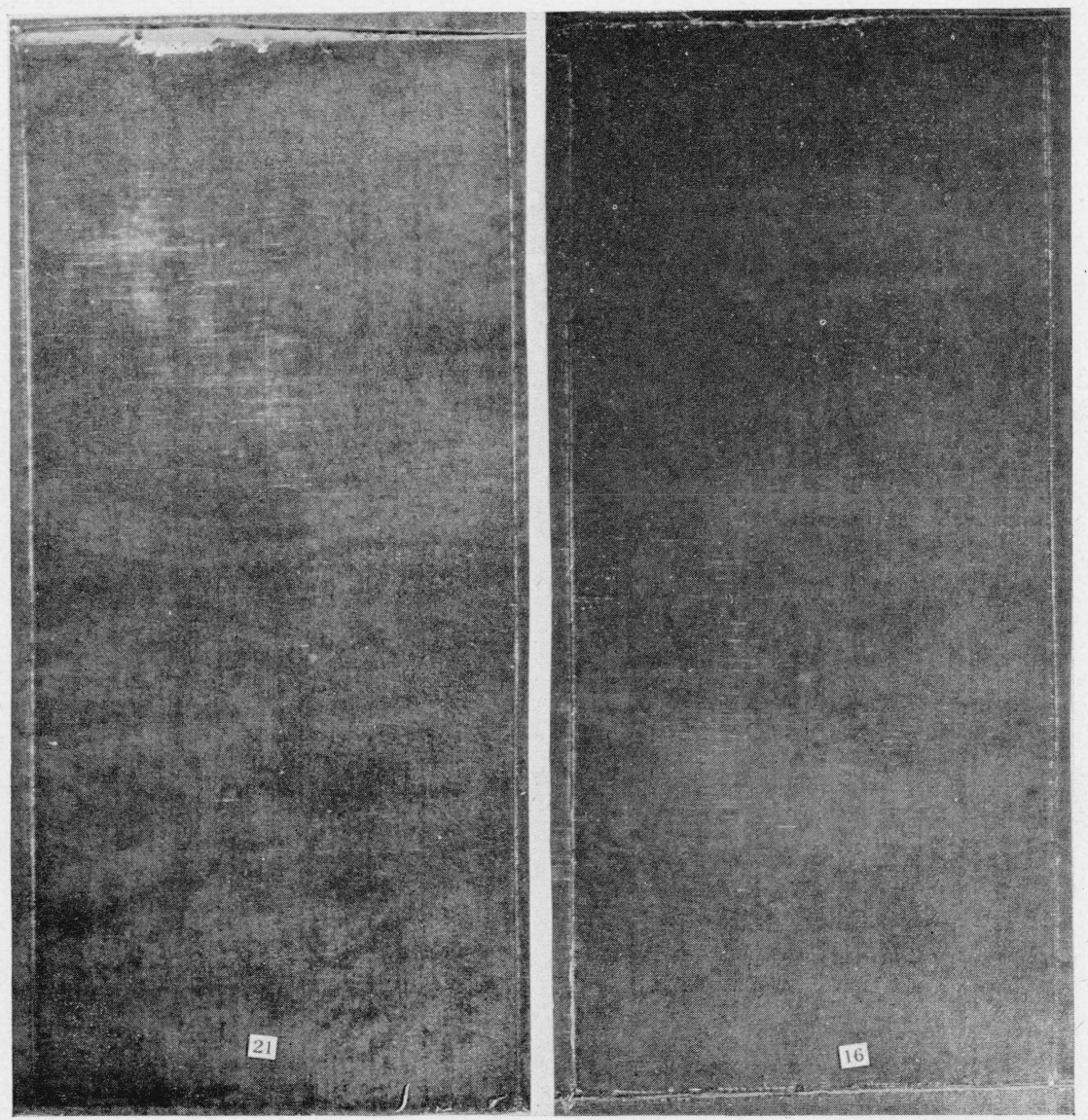

Figure 22.-Carpets 21 and 16 after 162 weeks of service wear; $S$ indices, 162 and 169 weeks, respectively. 

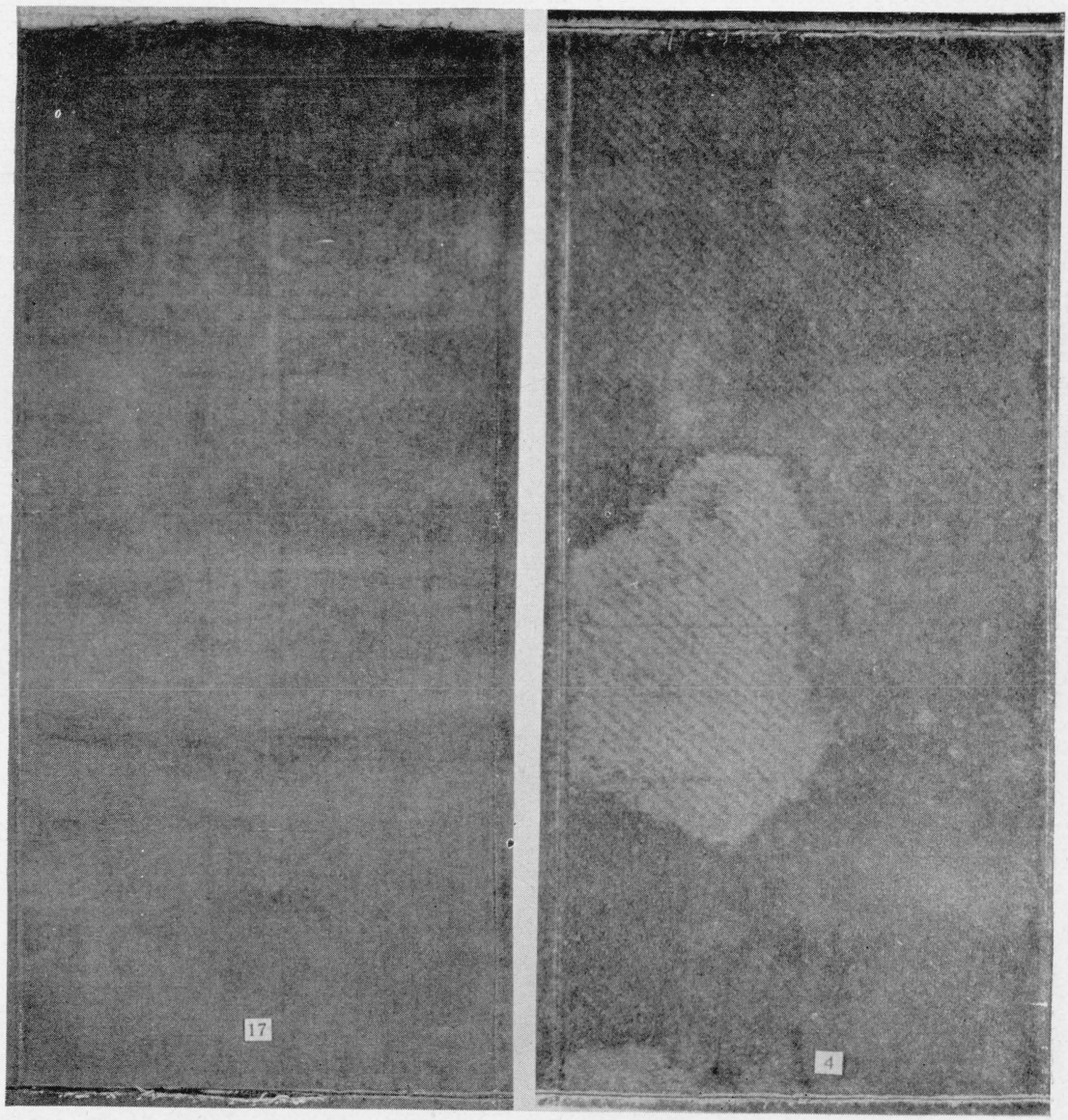

FIGURE 23.-Carpets 17 and 4 after 162 weeks of service wear; $S$ indices, 171 and 171 weeks, respectively. 

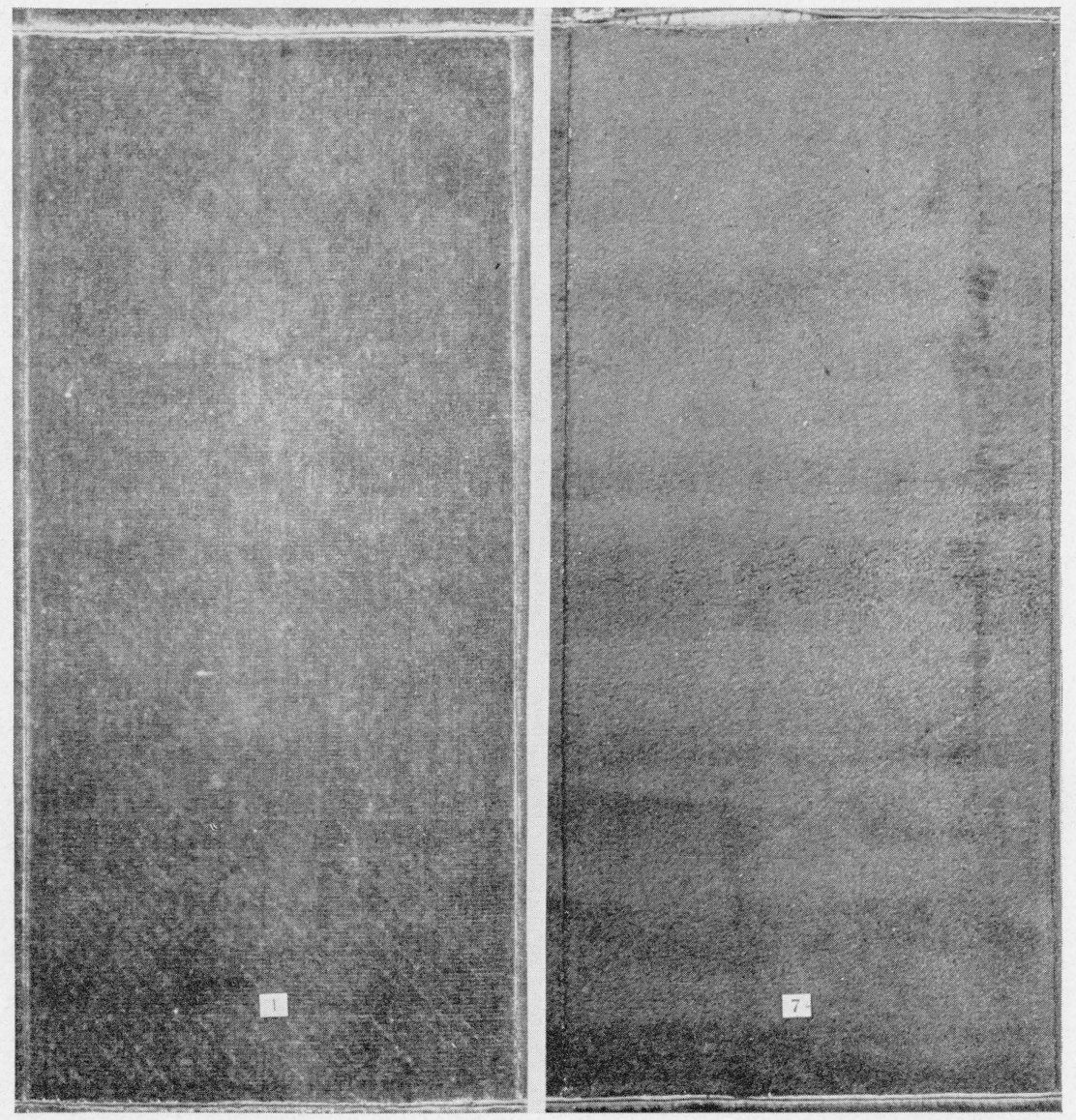

Figure 24.-Carpets 1 and $\%$ after 162 weeks of service wear; $S$ indices, 205 and 220 weeks, respectively. 

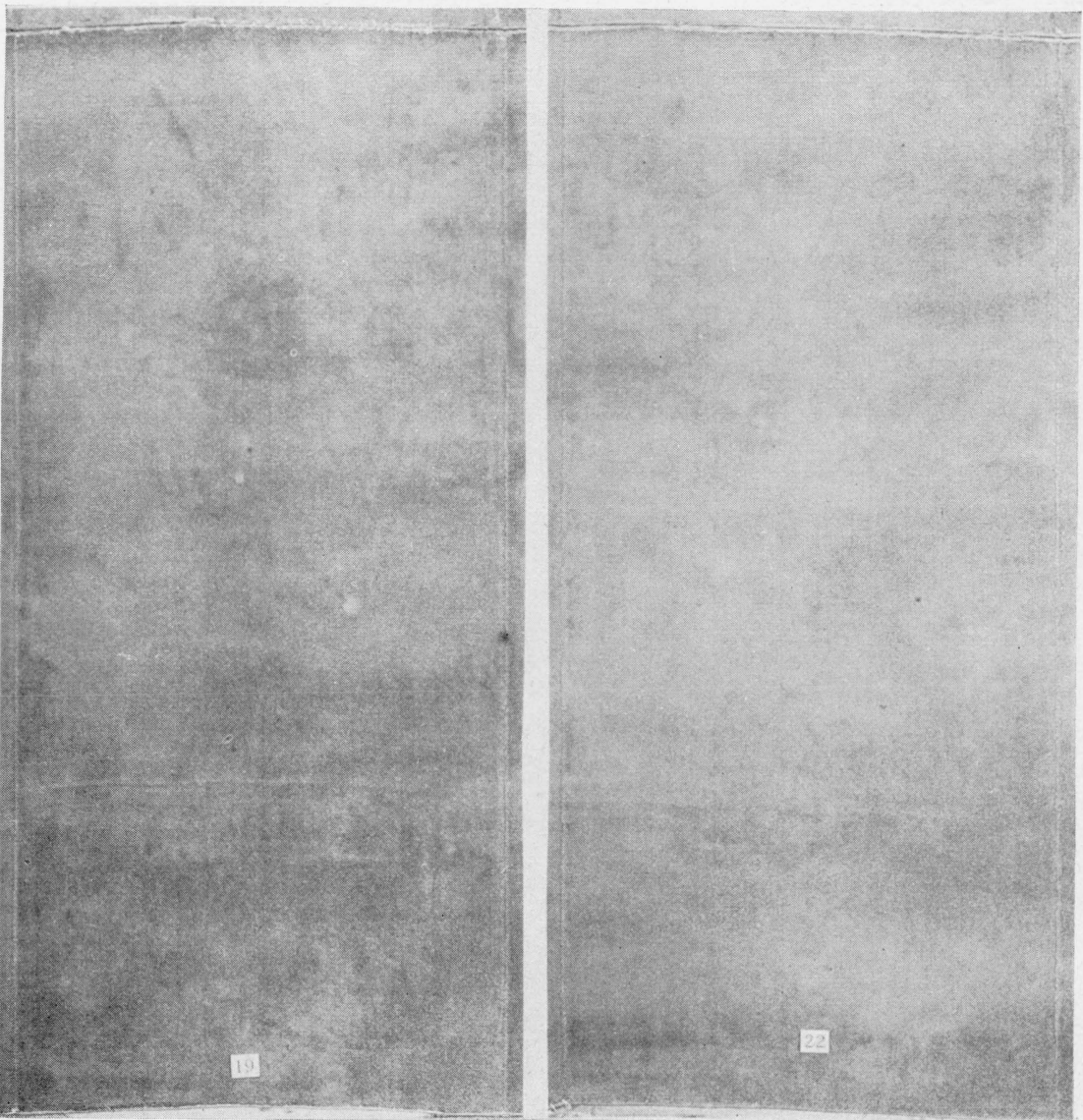

Figure 25.-Carpets 19 and 22 after 162 weeks of service wear; $S$ indices, 230 and 239 weeks, respectively. 


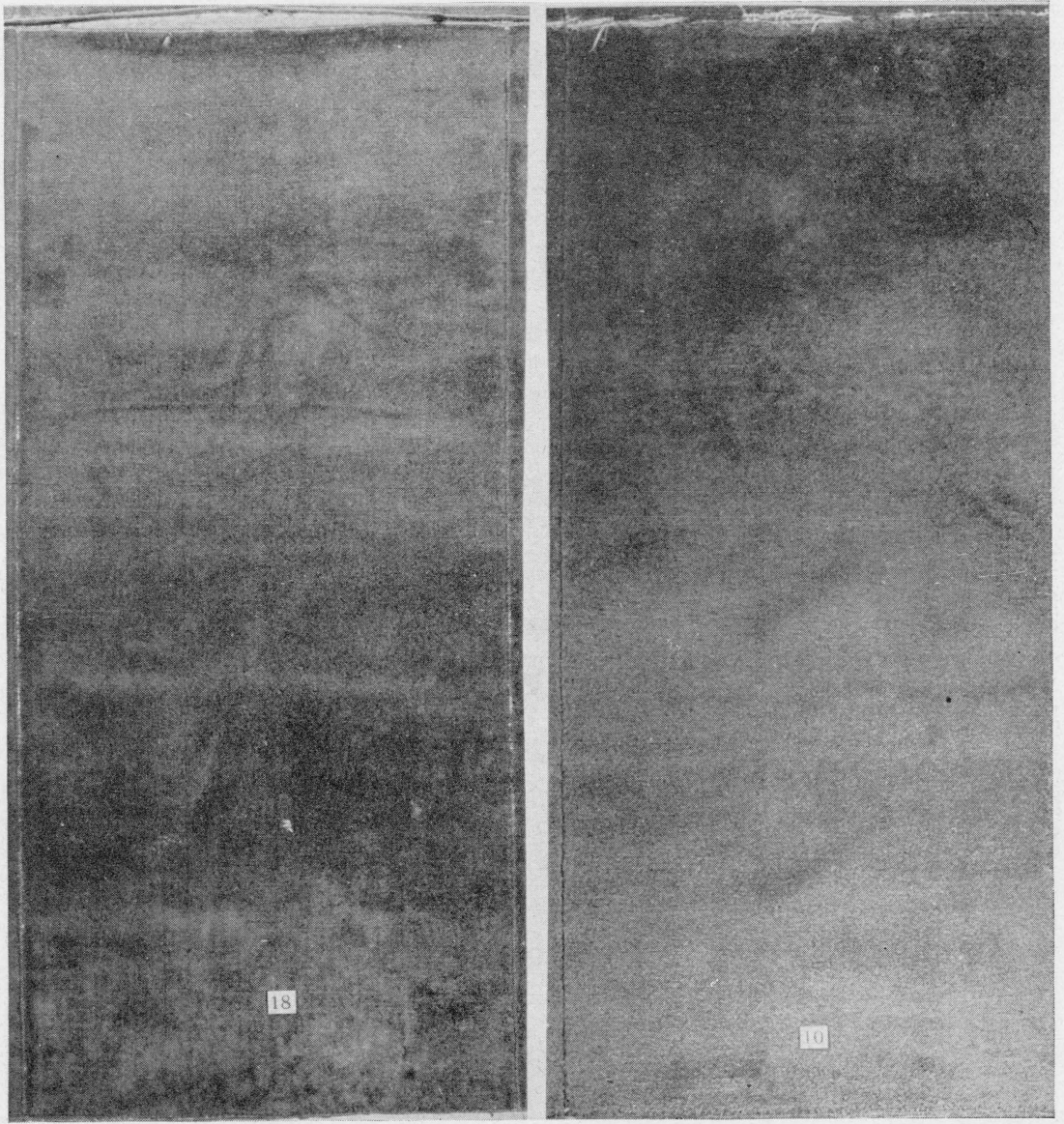

Figure 26.-Carpets 18 and 10 after 162 weeks of service wear; S indices, 245 and 260 weeks, respectively. 


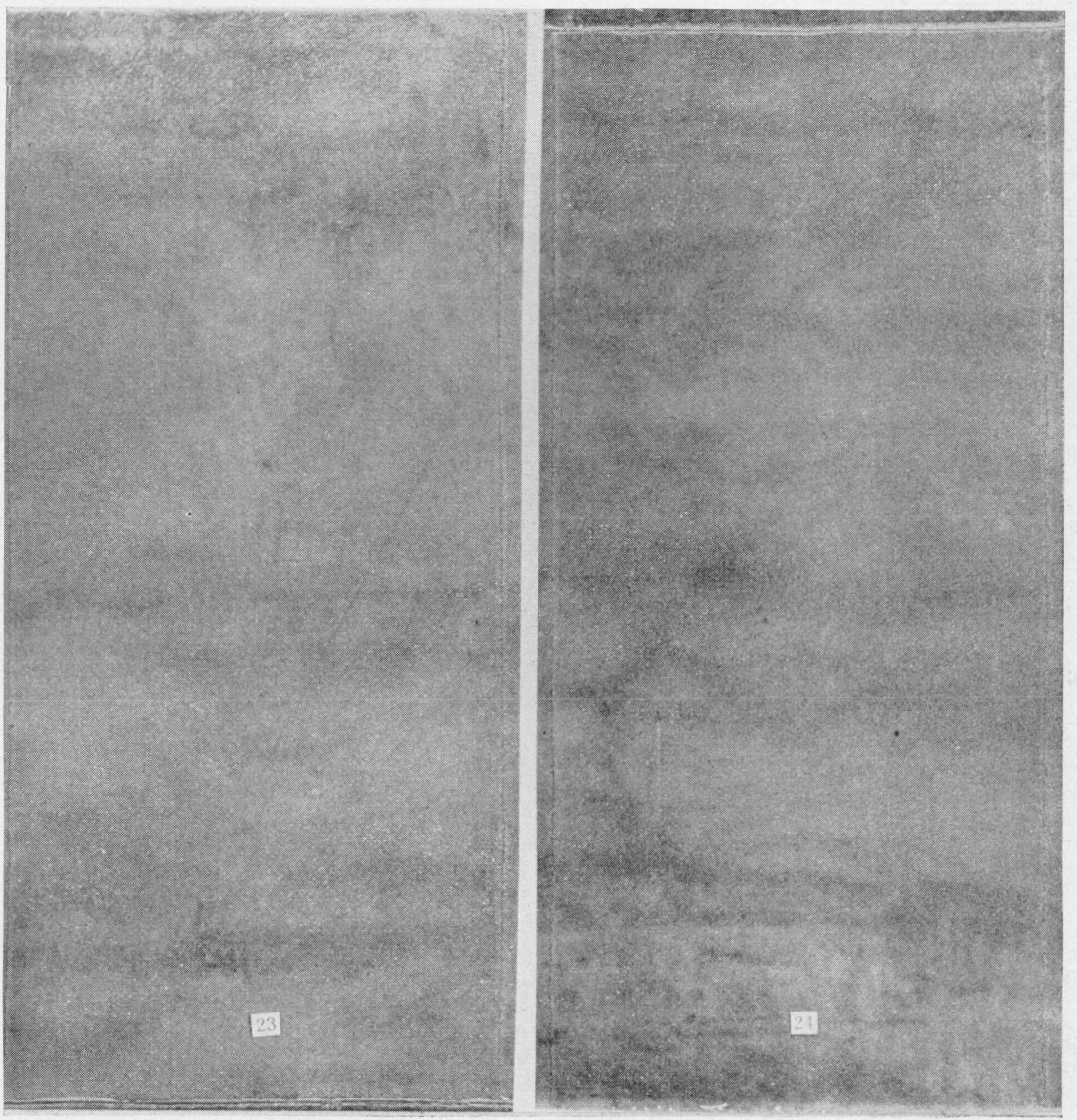

FIGURE 27.-Carpets 23 and 24 after 162 weeks of service wear; $S$ indices, 280 and 304 weeks, respectively. 

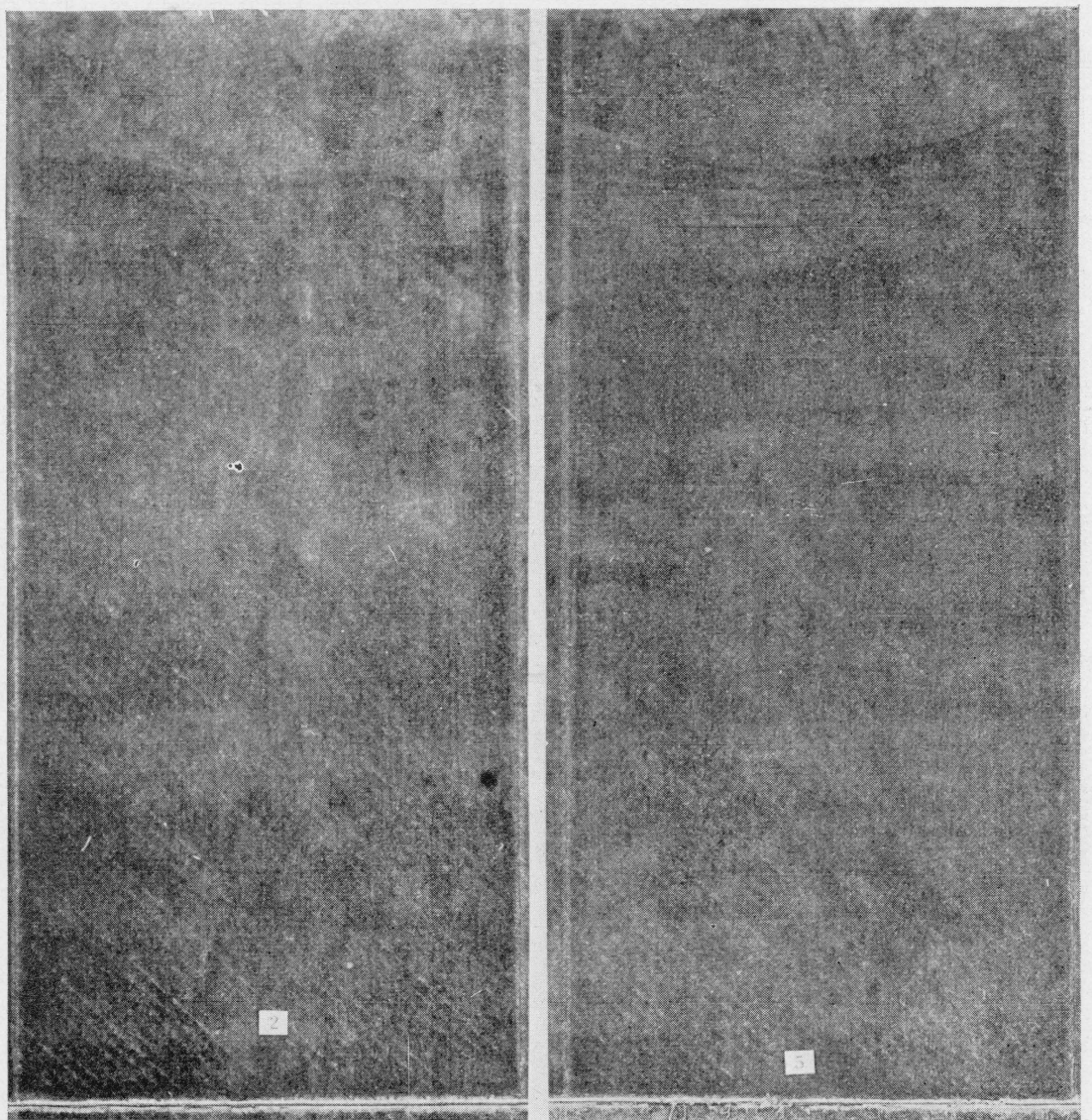

Figure 28.-Carpets 2 and 5 after 162 weeks of service wear; $S$ indices, 308 and 368 weeks, respectively. 

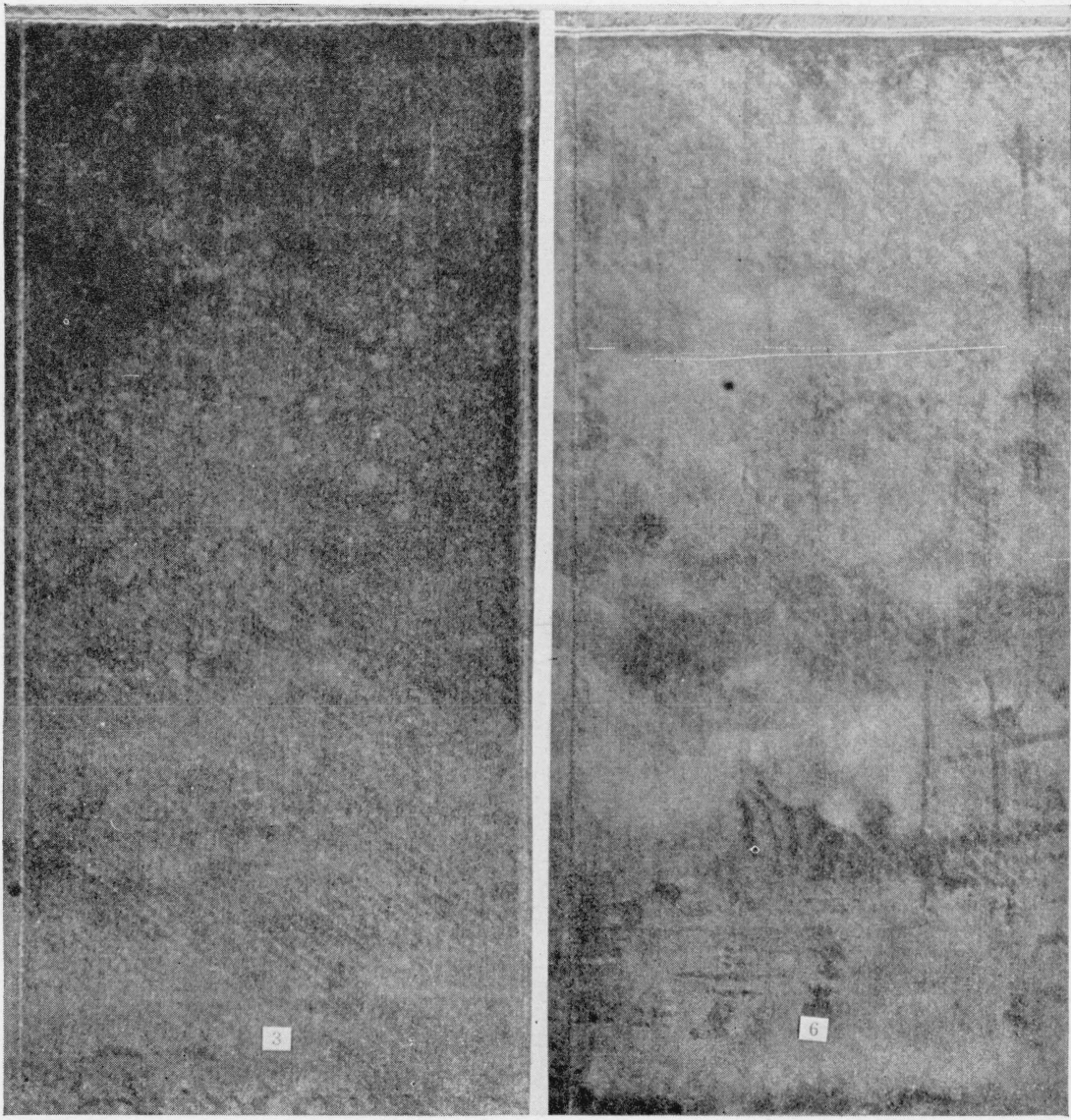

Figure 29.-Carpets 3 and 6 after 162 weeks of service wear; $S$ indices, 370 and 398 weeks, respectively. 


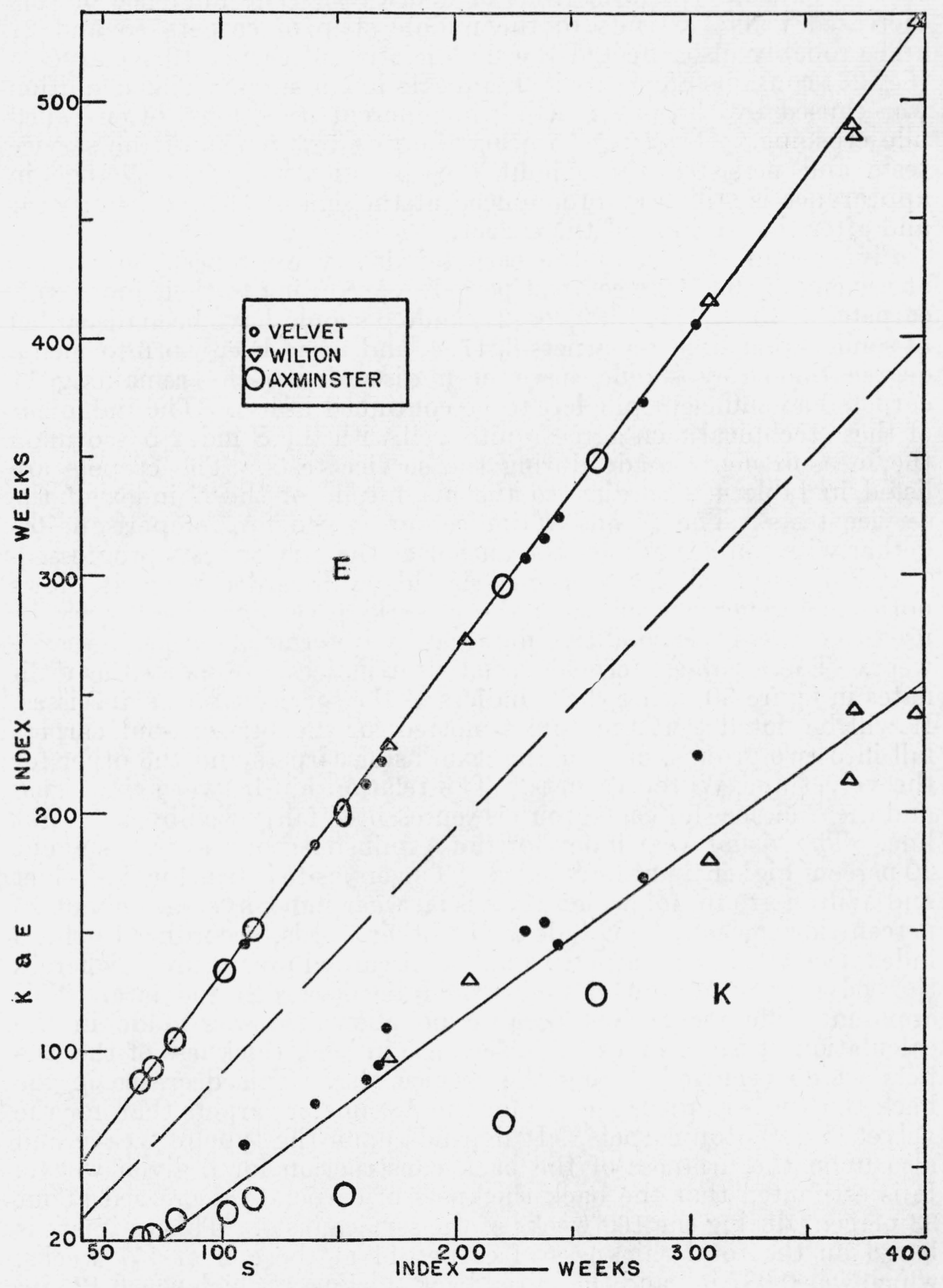

Figure 16.-Relationship between $S$ and $K$ and $E$ indices of carpets obtained in the service wear tests. 


\section{Journal of Research of the National Bureau of Standards}

The outside edges of the side strips showed in general less wear because the traffic was definitely less along the walls of the corridor. A few carpets showed more wear in one of their outside strips because of the increased traffic in front of doorways. The influence of this increased traffic extends to the middle strip of carpets 15 and 21 and probably also affected the middle strip of carpet 19. Carpet 4 (fig. 23) contains an area which appears like a stain. This condition was caused by the pile matting in different directions, often called pile crushing. It occurred during the first few weeks of the service tests and persisted throughout the investigation. The change in appearance is still very pronounced at the end of 162 weeks of wear and after the washing of the carpet.

Five technical men of the carpet industry examined the carpets at the end of the 162-week test period. According to their judgment, carpets $14,13,12,11,15,9,20,21$, and 16 should have been discarded at some earlier time. Carpets 8, 17, 4, and 1 had been worn to such a degree that they should have been discarded. The remaining 11 carpets had sufficient pile left to be continued in use. The judgment of these technical men agrees quite well with the $S$ index based upon the measurements made during the service tests. The carpets are listed in table 4 according to the magnitude of the $S$ index of the service tests. The $K$ and $E$ indices are 'given for comparison, together with notes made by the author as the service tests progressed.

If it is assumed that a carpet should be discarded when it shows noticeable general wear, then the remarks given in table 4 may be used to express this condition numerically in terms of weeks of service wear. These values, termed visual wear indices, are plotted as ordinates in figure 30 , using the $S$ indices of the service tests as abscissas. It will be noted that the points plotted for the 14 worn-out carpets fall into two groups, one for the Axminster carpets and the other for the velvet and Wilton carpets. The relationship between the visual and the $S$ indices for each group is represented fairly well by a straight line. The visual wear index for the Axminster group averages about 20 percent higher than the $S$ index. The opposite is true for the velvet and Wilton group, for which the visual wear index averages about 25 percent lower than the $S$ index. In other words, according to the $S$ index, the Axminster carpets would be discarded prematurely, whereas the velvet and Wilton carpets would be discarded too late. This apparent difference arises because no allowance was made in the calculation of the $S$ index for a decrease in back thickness of the carpets which occurred during the service test. This decrease in the back thickness is much greater for the Axminster carpets than for the velvet and Wilton carpets. It depends upon the type of weave and also upon the firmness of the back construction for a given weave. It is estimated that the back thickness of carpet 14 decreased about 33 percent during the 162 weeks of the service tests. This estimate is based on the total thickness of carpet 14 at the end of 157 weeks, which was 0.087 in., and the initial back thickness, which was 0.125 in. This estimate is probably low as there was still some pile remaining at the end of 162 weeks, as is shown in figure 17. If an allowance is made for this decrease in the back thickness, the corrected $S$ index is about 125 instead of 65 weeks. If this value is plotted against the visual wear index of 90 weeks in figure 30 , the point falls very 
nearly on the straight line for the velvet and Wilton group. If similar allowances are made for carpets 13 and 12 , the corrected $S$ indices

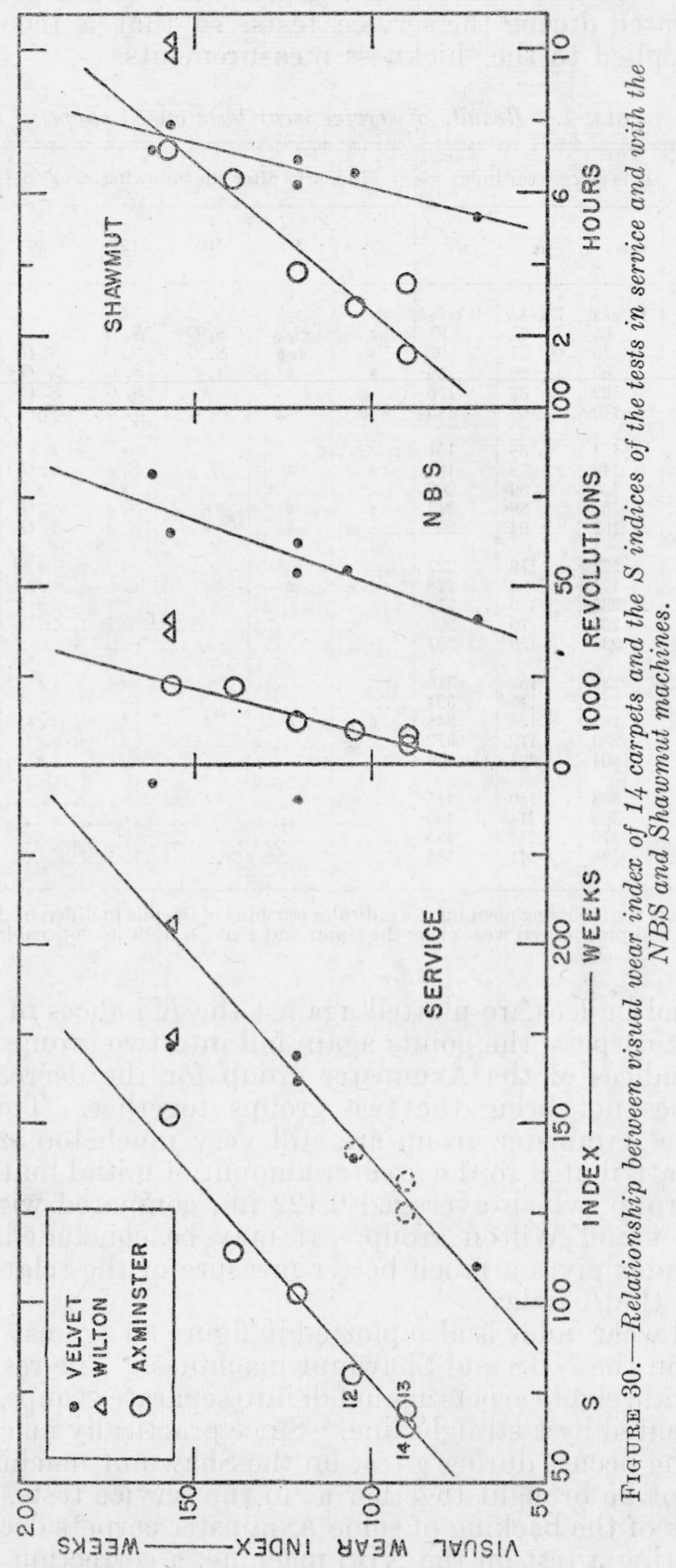

become about 130 and 150, respectively, instead of 70 and 80 weeks. The points for these values also fall very nearly on the straight line 
for the velvet and Wilton groups. This separation of the carpets into two groups may be attributed, therefore, to the decrease in the back thickness of the Axminster carpets. Unfortunately this decrease could not be evaluated during the service tests, so that a true correction cannot be applied to the thickness measurements.

TABLE 4.- Results of service wear tests on 24 carpets

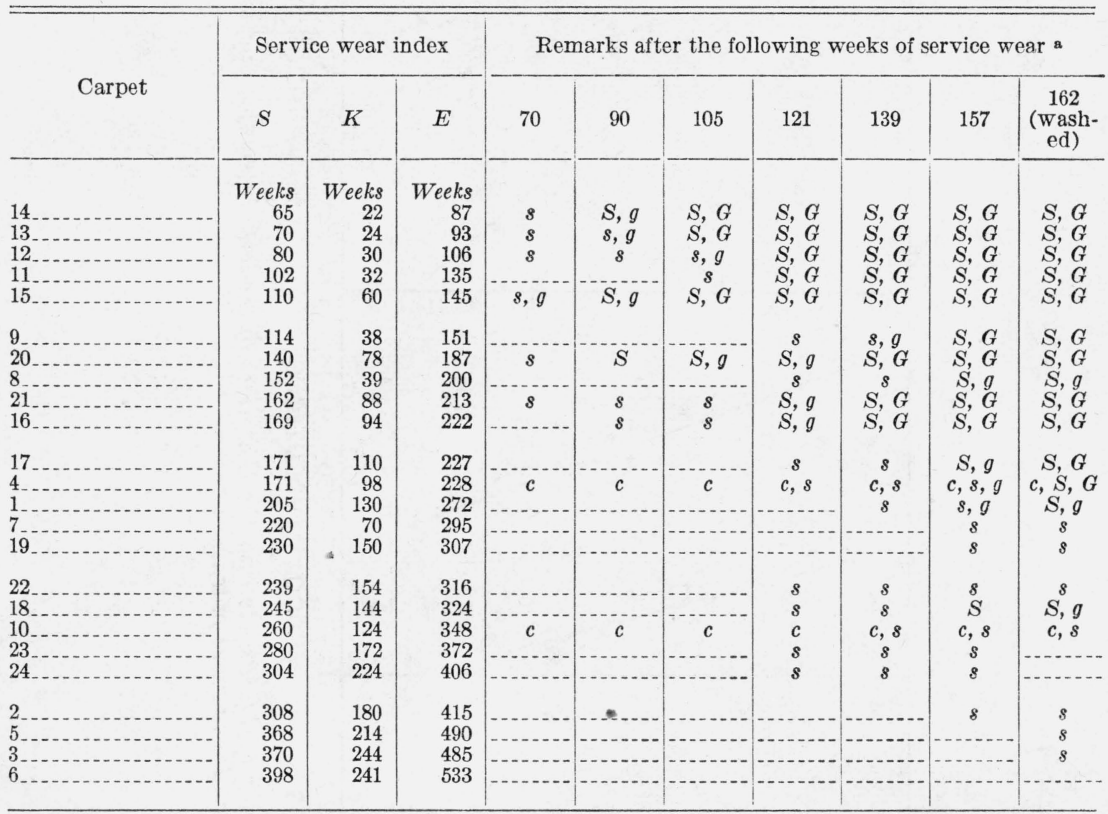

a The symbols have the following meanings: $c$ indicates crushing of the pile in different directions; $s$ and $S$ indicate noticeable and pronounced wear along the seam: and $g$ and $G$ indicate noticeable and pronounced general wear.

If the visual indices are plotted against the $K$ indices of the service tests of these carpets, the points again fall into two groups. Correcting the $K$ indices of the Axminster group for the decrease in back thickness does not bring the two groups together. The corrected results of the Axminster group are still very much too small. This difference is attributed to the greater amount of initial matting for the Axminster group, which averaged 0.122 in., compared with 0.076 in. for the velvet and Wilton group. It may be concluded, therefore, that the $S$ index gives a much better measure of the relative wear of carpets than the $K$ index.

The visual wear index is also plotted in figure 30 against the $S$ index of the tests on the NBS and Shawmut machines. The results for the Axminster and velvet carpets again fall into separate groups, each group being represented by a straight line. Since practically no compression of the backing occurs during a test on the Shawmut machine, the two groups cannot be brought together as in the service tests. Although the thickness of the backing of some Axminster carpets decreased considerably during a test on the NBS machine, a correction for this decrease also does not bring the two groups into coincidence. The ex- 
planation for the difference between the Axminster and velvet carpets is not apparent but presents a very puzzling problem. It could be assumed that the results of the service tests of the velvet carpets were low because the amount of traffic was greater than for the Axminster carpets. However, since the difference in wear of the two strips at the ends of the corridor was very slight, the $S$ indices being 138 and 150 weeks (fig. 20), this explanation does not seem very probable. Localized variation in traffic along the length of the corridor, due to doorways and other causes, can be ruled out as a contributing cause, since there is little scattering of the points for either the velvet or the Axminster groups in figure 30. The difference in wool blend, dyeing, and yarn structure between the carpets of the Axminster and velvet weaves should have the same effect on the visual wear index and on the $S$ index of the laboratory tests, unless these factors greatly influenced the appearance of the carpets during the service tests. Appearance is, of course, not taken into consideration when the wear index is based upon thickness measurements. A difference in the nature of the wear on the fibers during the laboratory tests is another factor which could contribute to the observed difference.

\section{COMPARISON OF SERVICE, NBS, AND SHAWMUT TESTS ON 24 CARPETS}

The $S$ index of the service tests of 24 carpets is plotted against the $S$ index of the tests on the NBS machine in figure 31 . The over-all correlation if fair. The correlation for the carpets of each weave is good, with the carpets of each weave forming a distinct group. The Wilton group falls between the Axminster and velvet groups. The lines for the three groups are nearly parallel and intercept the coordinate axes near the origin.

The $S$ index of the service tests is also plotted against the $S$ index of the tests on the Shawmut machine in figure 31 . The over-all correlation is fair. The correlation for the carpets of each weave is good, with the carpets of each weave forming a distinct group. The lines for the Wilton and velvet groups are nearly parallel, but their intercepts with the coordinate axes are not near the origin. The slope of the line for the Axminster group is, however, very different and the intercepts of the line with the coordinate axes are near the origin.

The $S$ index of the tests on the NBS machine is plotted against the $S$ index of the tests on the Shawmut machine in figure 32 . The over-all correlation is poor. The correlation for the carpets of each weave is good, with the carpets of each weave forming a distinct group. The lines for the Wilton and velvet groups are nearly parallel, but their intercepts with the coordinate axes are not near the origin. The slope of the Axminster group is, however, very different, and the intercepts of the line with the coordinate axes are near the origin.

The relative wear of these carpets when tested on the Shawmut and NBS machines depends very greatly upon some factor which is different for the three weaves. In addition to the fundamental differences between the three weaves, the carpets of one weave probably differ from those of another weave in wool blend, dyeing, and yarn structure, since the carpets of each weave were made by a different manufacturer. It is likely that these factors influence the results differently in each of the three types of tests. The present data are not sufficient to permit a detailed study of this problem. 
358 Journal of Research of the National Bureau of Standards

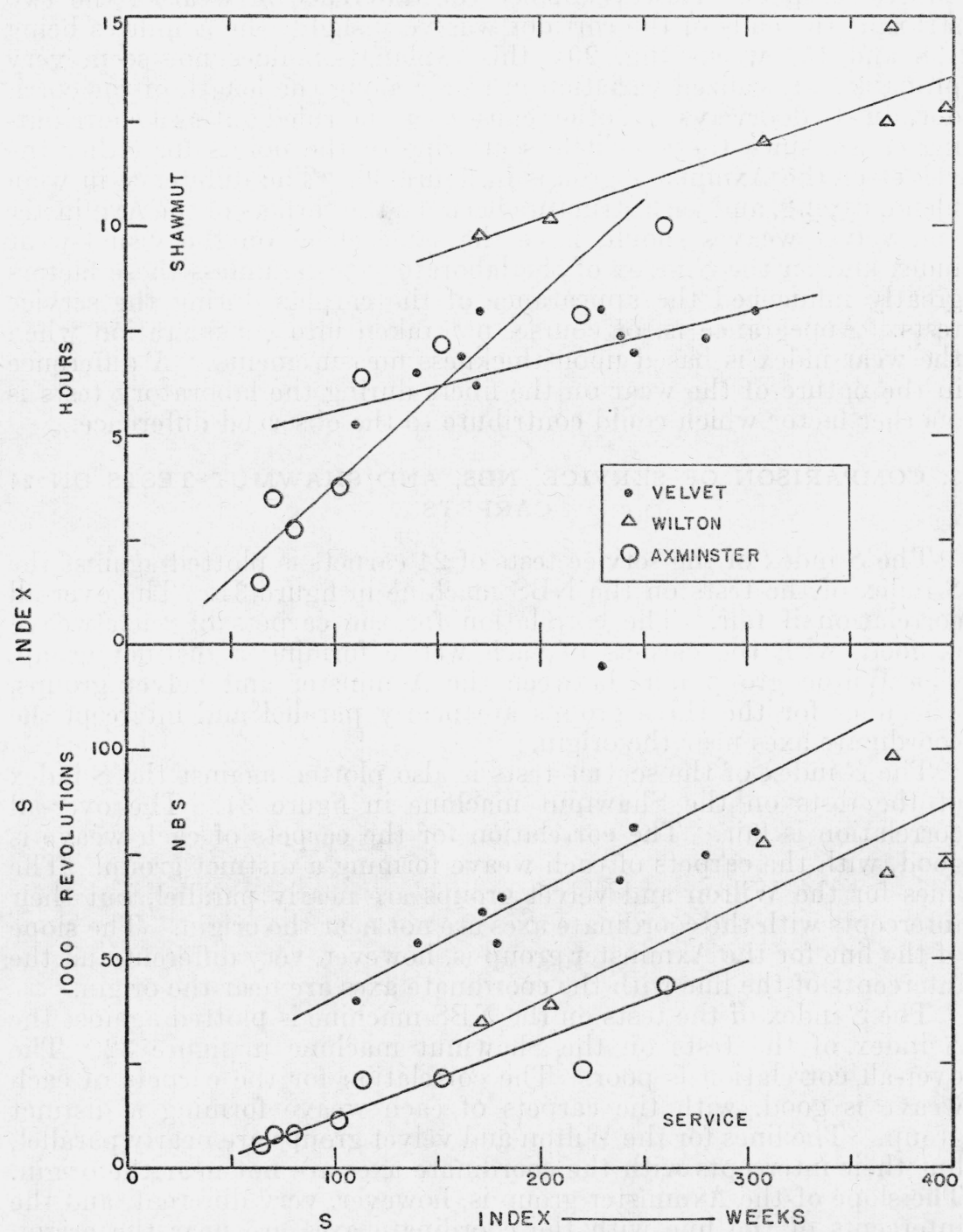

FIGURE 31.-Relationship between the $S$ index of 24 carpets of the service tests and the $S$ indices of the tests with the NBS and Shawmut machines. 


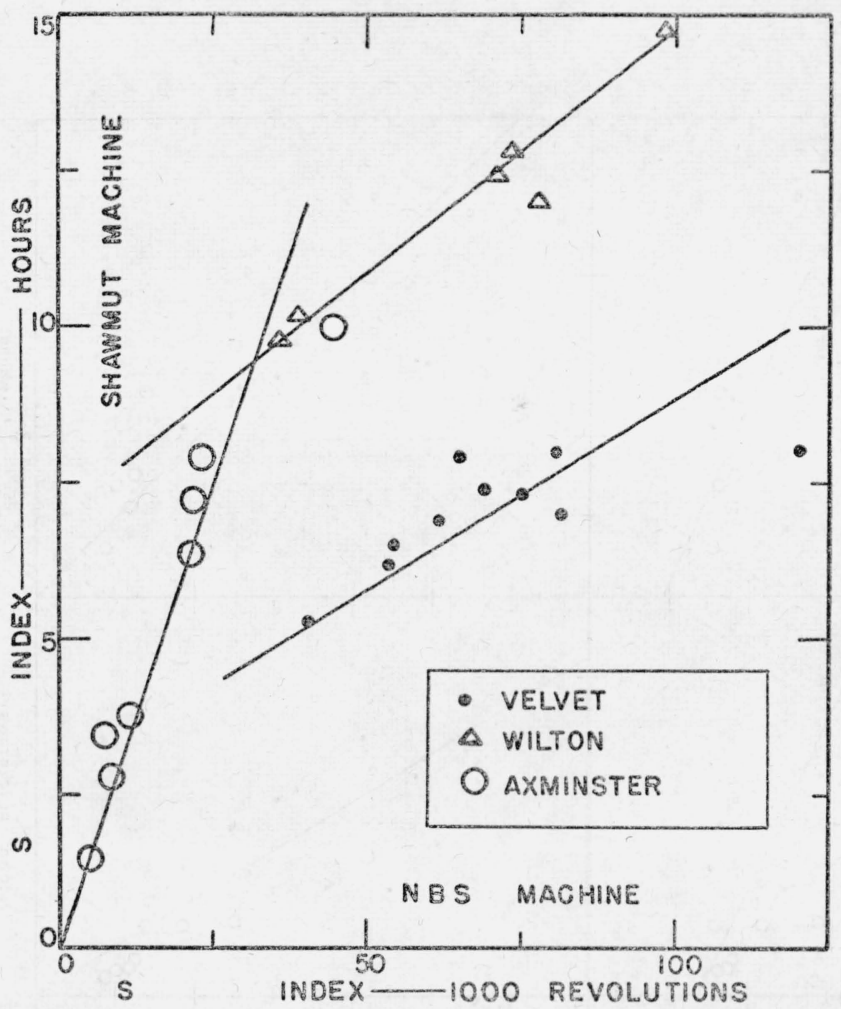

FIGURE 32.-Relationship between the $S$ indices of 24 carpets tested with the NBS and Shawmut machines.

\section{COMPARISON OF WEAR TESTS WITH ANALYSES OF CARPETS}

The products $D^{2} H$ and $D H$, where $D$ and $H$ are the density and height of pile, respectively, have been used as a criteria of the relative wear of carpets. The $S$ index of the service tests is plotted against $D^{2} H$ and $D H$ in figure 33 . The $S$ indices of the tests on the NBS and Shawmut machines are similarly plotted in figure 33. The correlation of the $S$ indices with $D^{2} H$ is good. There is some indication, however, that the correlation is affected by the type of weave or more likely by the wool blend used in the carpets of the three weaves. The correlation of the $S$ indices with $D H$ is also good, although the straight lines do not pass through the origin. There is again some indication that the correlation is affected by the type of weave, wool blend, dyeing, and yarn structure. A more thorough discussion of these factors is given in section 8 .

The 24 carpets of Wilton, velvet, and Axminster weaves were made in two series for each weave. In one series all the manufacturing details were kept constant except that the wire height (pile height) was varied systematically. In the other series, all the manufacturing details were kept constant except that the number of rows per inch (tufts per square inch) was varied systematically. The effect of an increase in pile height or in number of tufts per square inch may thus be evaluated. 


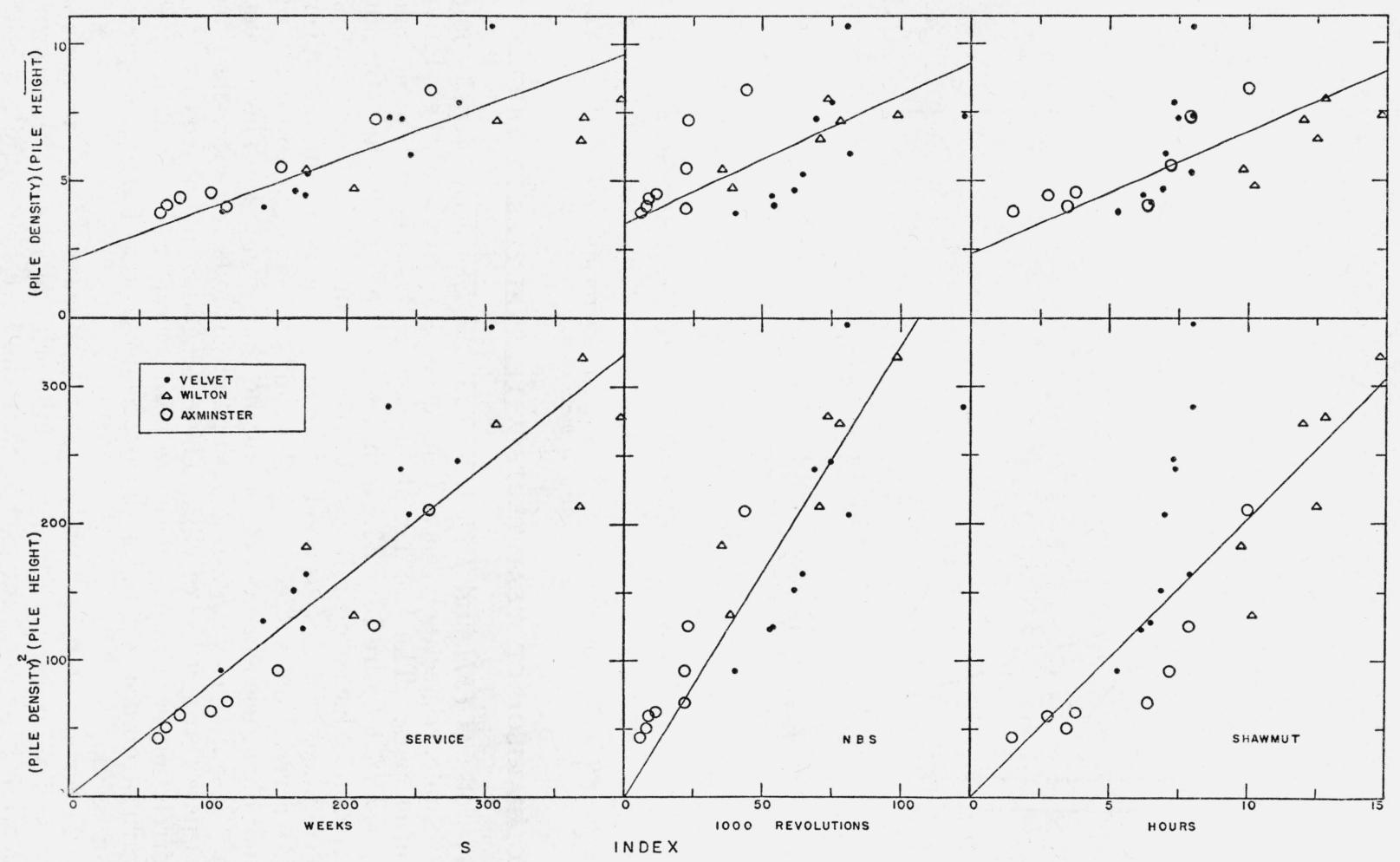

FIGURE 33.-Relationship between the $S$ indices of 24 carpets and the products $D^{2} H$ and $D H$, where $D$ and $H$ are density and height of pile, respectively. 
The percentage increase in the $S$ index of the service, NBS and Shawmut tests is plotted in figure 34 against the percentage increase in pile height. The points belonging to the same series are connected by straight lines. An increase of 100 percent in pile height produces an increase of about 75 percent in the $S$ index of the service tests. The corresponding increases in the $S$ indices for the tests on the same carpets with the NBS and Shawmut machines are only 25 and 15 percent, respectively. The laboratory wear tests apparently underestimate the effect of an increase in the height of the pile on the wear of the carpets.

The percentage increase in the $S$ index of the service, NBS and Shawmut tests is also plotted in figure 34 against the percentage increase
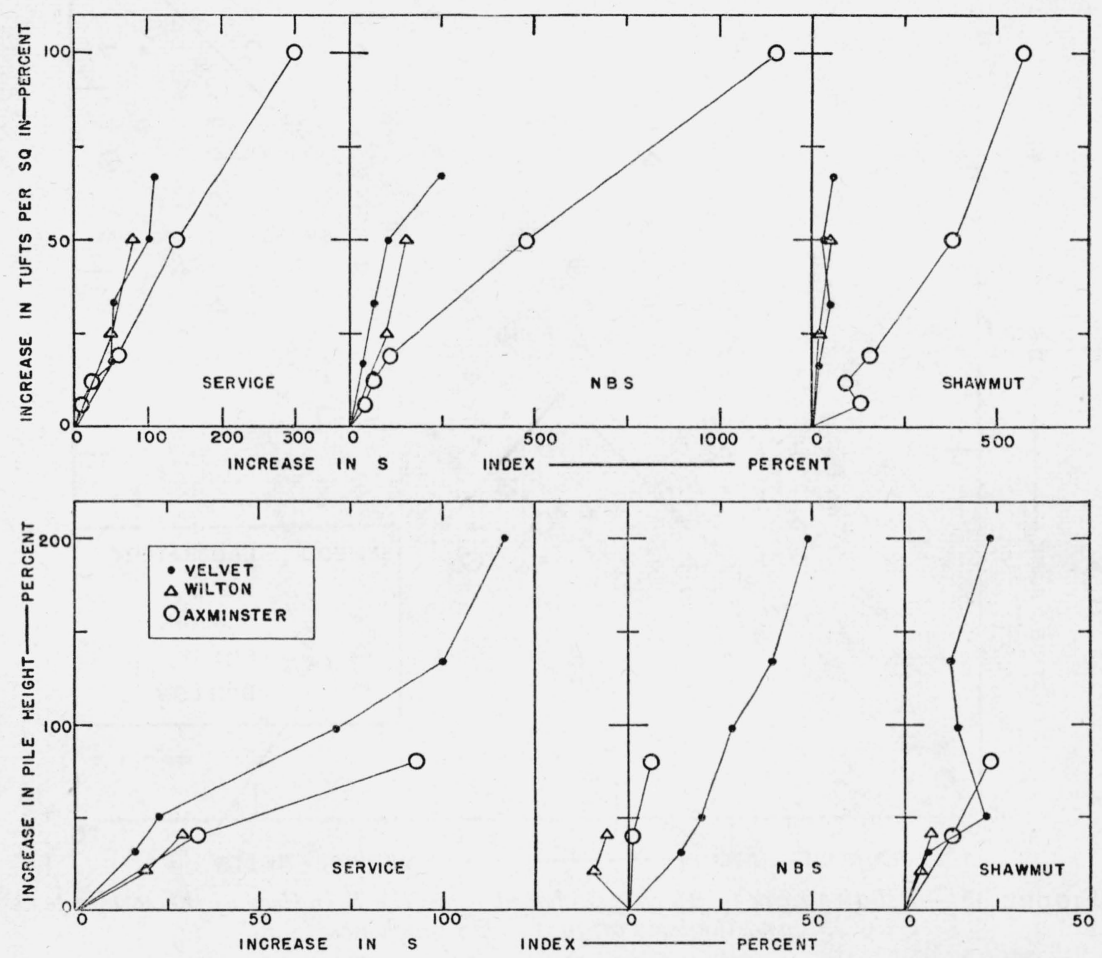

FIGURE 34.-Effect of pile height and number of tufts per square inch on the $S$ index.

in number of tufts per square inch. The points belonging to the same series are connected by straight lines. An increase of 100 percent in the number of tufts per square inch produces an increase of about 225 percent in the $S$ index of the service tests. This increase is three times the increase obtained for an increase of 100 percent in height of pile. The increases in the $S$ indices for the tests on the same carpets with the NBS and Shawmut machines depend very much upon the weave. The increase obtained with the Shawmut machine is about 100 percent for the Wilton and velvet weaves and about 600 percent for the Axminster weave. The increase obtained with the NBS machine is about 250 percent for the Wilton and velvet weaves 
and about 800 percent for the Axminster weave. The laboratory tests are probably unduly severe on carpets of low quality, and this may account for the large increase obtained for the Axminster carpets, since the percentage increases were computed on the basis of the $S$ index of an Axminster carpet of very low quality.

\section{INTERLABORATORY TESTS}

The 24 carpets which were subjected to service tests were also tested with the NBS machine in the laboratories of the Mohawk Carpet Mills,

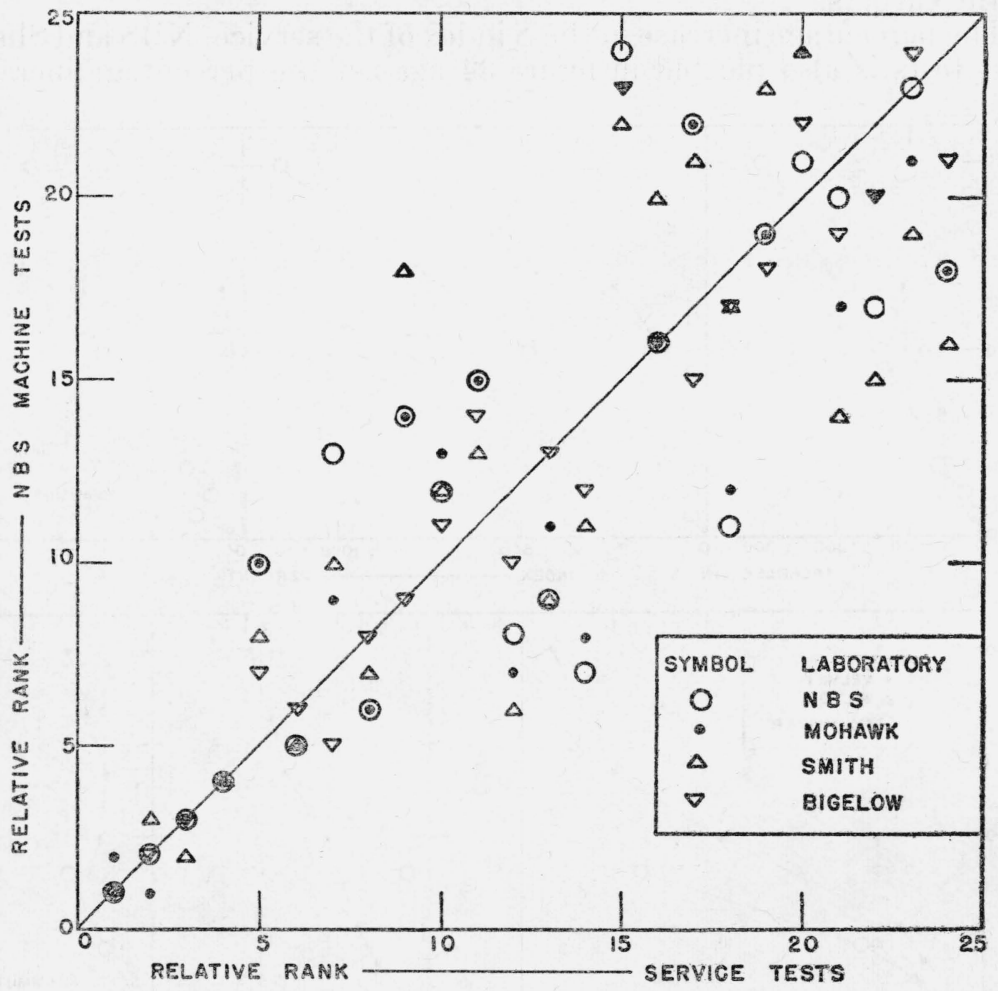

FIG URE 35.-Relative rank of 24 carpets based upon the $S$ indices of the service tests and the tests on four NBS machines.

Inc., the Alexander Smith \& Sons Carpet Co., and the Bigelow Sanford Carpet Co., and with the Shawmut machine, in the laboratories of the Alexander Smith \& Sons Carpet Co. and the Bigelow Sanford Carpet Co. Using the $S$ index of the service test as a basis for arranging the carpets in a relative order, the Kendall rank correlation coefficients were computed for each laboratory and test method. These coefficients ranged in value from 0.62 to 0.85 for the NBS machine and from 0.71 to 0.78 for the Shawmut machine. The corresponding coefficients for $D^{2} H$ and $D H$ were 0.78 and 0.71 , respectively. All these coefficients show a highly significant correlation with the $S$ index of the service tests. The relative rank of the 24 carpets according to the $S$ index of the tests with the NBS machine in the different 
laboratories is plotted in figure 35 against the relative rank according to the $S$ index of the service tests. Figures 36 and 37 give similar data for the Shawmut machine and carpet analysis, respectively. It is apparent from these figures that the general correlation of the relative rank of the carpets tested in the laboratories with the relative rank of the carpets of the service tests is very good.

Although the results obtained by the different laboratories apparently evaluated the carpets in much the same relative order as the service test, the numerical values which were obtained differed greatly. These differences are shown by the ratios of the results obtained at

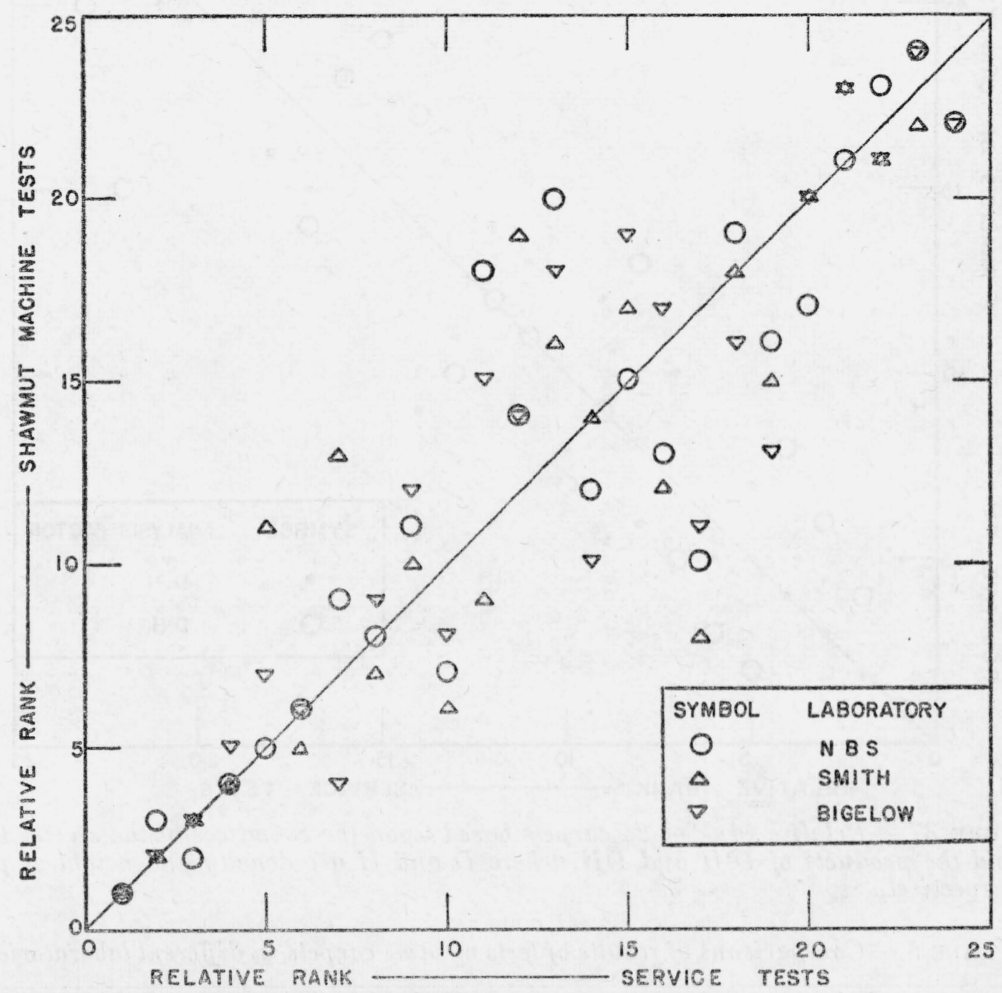

FiguRE 36.-Relative rank of 24 carpets based upon the $S$ indices of the service tests and the $E$ indices of the tests on three Shawmut machines.

the National Bureau of Standards to the results obtained in each of the cooperating laboratories. The average ratio and coefficient of variation for each laboratory and testing machine are given in table 5 . The large systematic differences obtained with the NBS machine are probably due to a lack of uniformity in the testing procedure. The tests at the National Bureau of Standards were carried to completion on each sample tested; that is, each test was continued until the pile was worn off. This procedure was not followed generally in the cooperating laboratories. If a test is discontinued too soon and the wear indices are arrived at by extrapolation of the wear-test curve, then values which are too low will be obtained. The testing procedure in 
this respect varied less in the case of the Shawmut machine, since this machine is set to stop automatically when the thickness of the carpet is reduced to its back thickness. The closer agreement between laboratories for this machine is apparent.

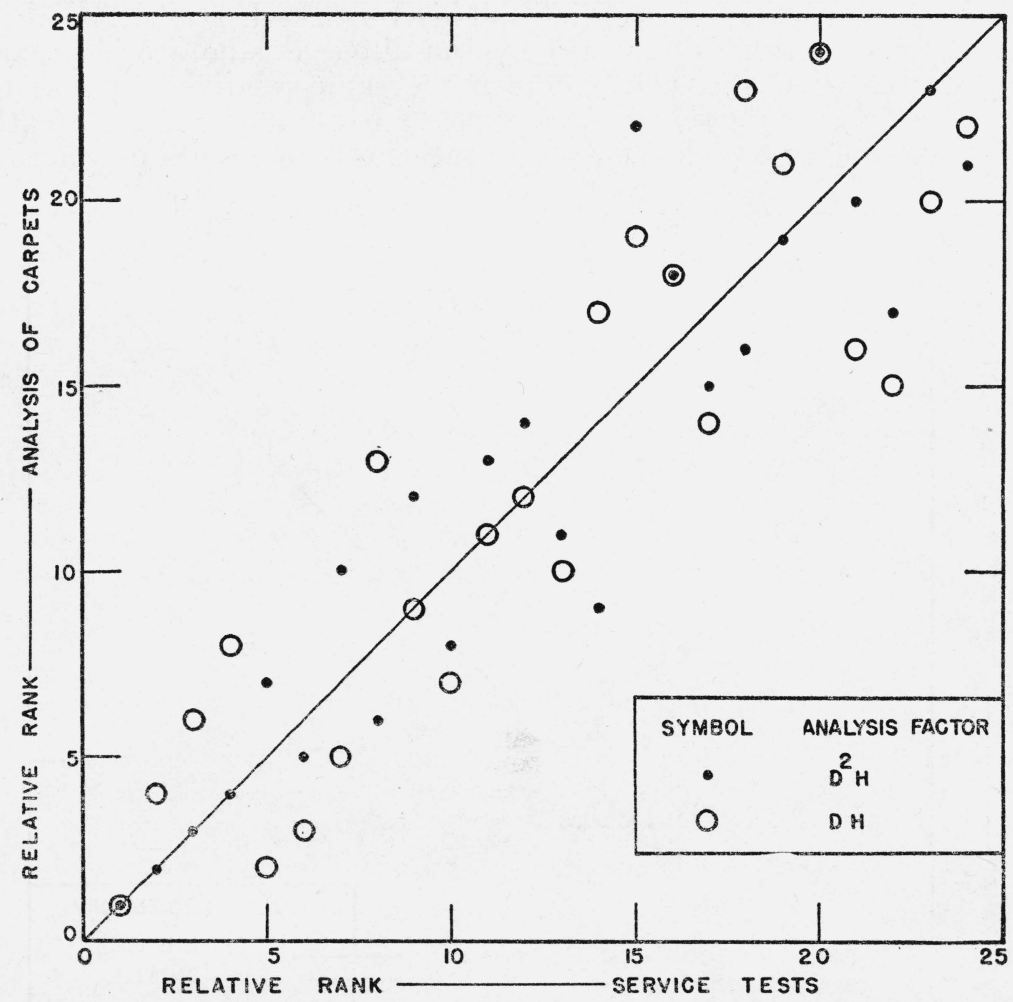

FIGURE 37.-Relative rank of 24 carpets based upon the $S$ indices of the service tests and the products of $D^{2} H$ and $D H$, where $D$ and $H$ are density and height of pile, respectively.

TABLE 5.-Comparisons of results of tests of same carpets in different laboratories

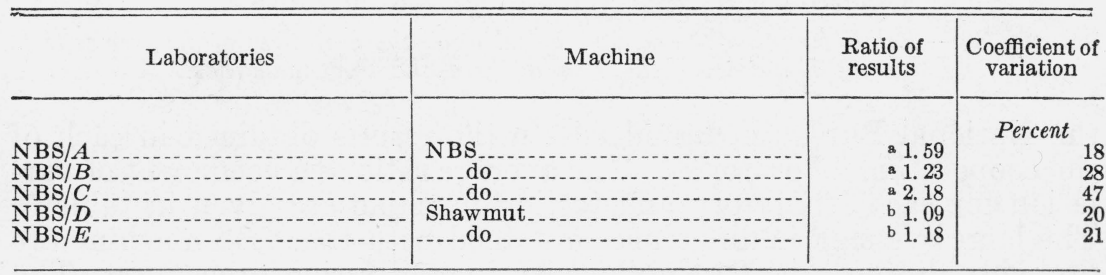

- Ratio is based upon $S$ indices.

b Ratio is based upon $E$ indices. 
The coefficient of variation is large for each machine and laboratory. One factor which probably contributes greatly to this variation is the variation in back thickness obtained for the same carpets in the different laboratories. Such a variation is critical since it affects the wear index directly and considerably. The difference in back thickness for the same carpet varied as much as 15 percent between laboratories. The large coefficient of variation of the ratios of the tests at the National Bureau of Standards to the tests at laboratory $C$ with the NBS machine results from some factor which caused a large systematic difference in the results of the 10 velvet carpets. This fact is brought out by the different ratios obtained for the three weaves-namely, $1.77,1.50$, and 2.97 for the Wilton, Axminster, and velvet carpet series, respectively. The large ratios result because the $S$ indices obtained in laboratory $C$ were low owing to the extrapolation of the wear-test curves. Whether the much larger ratio for the velvet carpets is entirely due to extrapolation or whether it is due to another cause, such as a change in the leather, can not be determined from the available data.

The relationship of the results of the tests on the 24 carpets in the different laboratories and with the different machines to the results of the service tests for the three weaves is shown by the data given in table 6 . This table contains the average and standard error of the ratios of the $S$ indices of the service wear tests of carpets to those of the laboratory tests for the Wilton, Axminster, and velvet weaves. Since the $S$ indices for the Shawmut machine were not obtained at laboratories $D$ and $E$, the ratios given for this machine are based upon the $E$ indices. The ratios for the tests with the Shawmut machine at the National Bureau of Standards are based upon both the $S$ and $E$ indices. The ratios for $D^{2} H$ and $D H$ are also given for comparison. The systematic difference between the results obtained at laboratory $C$ and the other laboratories with the NBS machine is again very evident. The rather large discrepancies in the ratios for the different weaves obtained in each laboratory or for the same weave obtained in the different laboratories indicate the absolute need for closer standardization of the testing machines and procedures. The weighted averages of the different laboratories, with the one exception, are in good agreement for each testing machine. This good agreement merely indicates that the errors arising from nonuniform wear of the service wear tests, and due to variations in the laboratory wear tests and in the back thickness determinations, are compensated for in the weighted averages. It may be concluded that the results of adequately standardized laboratory wear tests can be used safely to judge the probable relative wear of carpets for the same use. 
TABLE 6.-Average and standard error of the ratios of the $S$ indices of the service wear of carpets to those of the laboratory for the Wilton, Axminster, and velvet weaves

\begin{tabular}{|c|c|c|c|c|c|c|c|c|c|c|}
\hline \multirow{2}{*}{ Weave } & \multicolumn{4}{|c|}{ NBS machine } & \multicolumn{4}{|c|}{ Shawmut machine } & \multirow{2}{*}{$D^{2} H$} & \multirow{2}{*}{$D H$} \\
\hline & NBS & Lab. $A$ & Lab. $B$ & Lab. $C$ & NBS & NBS a & Lab. $D^{\mathrm{a}}$ & Lab. $E^{a}$ & & \\
\hline 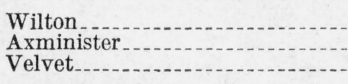 & $\begin{array}{l}4.6 \pm 0.3 \\
7.8 \pm 0.8 \\
3.0 \pm 0.2\end{array}$ & $\begin{array}{l}7.4 \pm 0.9 \\
4.2 \pm 1.7 \\
4.4 \pm 0.3\end{array}$ & $\begin{array}{l}7.4 \pm 0.3 \\
6.9 \pm 1.0 \\
3.5 \pm 0.2\end{array}$ & $\begin{array}{r}8.2 \pm 0.5 \\
11.4 \pm 1.1 \\
7.8 \pm 1.0\end{array}$ & $\begin{array}{l}2.5 \pm 0.2 \\
2.7 \pm 0.3 \\
2.9 \pm 0.2\end{array}$ & $\begin{array}{r}9.8 \pm 0.4 \\
13.3 \pm 1.8 \\
12.8 \pm 1.0\end{array}$ & $\begin{array}{r}9.9 \pm 0.9 \\
14.3 \pm 0.5 \\
13.7 \pm 1.3\end{array}$ & $\begin{array}{l}12.8 \pm 0.8 \\
14.1 \pm 1.0 \\
15.1 \pm 1.1\end{array}$ & $\begin{array}{l}1.3 \pm 0.1 \\
1.5 \pm 0.2 \\
1.1 \pm 0.1\end{array}$ & $\begin{array}{l}44 \pm 4 \\
23 \pm 2 \\
32 \pm 1\end{array}$ \\
\hline Weighted average.. & 5.0 & 5.1 & 5.6 & 9.1 & 2.7 & 12.2 & 13.1 & 14.2 & 1.3 & 32 \\
\hline
\end{tabular}

- These ratios are based upon the $S$ indices of the service wear tests and the $E$ indices of wear tests with the Shawmut machine. 


\section{EFFECT OF TYPE OF VACUUM-CLEANING SYSTEM ON THE SERVICE WEAR OF CARPETS}

After the 24 carpets had been in service for nearly 2 years they were soiled in appearance and considerable dirt was embedded in the pile. Because of this condition, it seemed advisable to change the cleaning system and procedure. Since this might result in a change in the rate of wear of the carpets, it was decided to use three types of cleaners and to study the effect of each on the rate of wear. The three types of cleaners selected for this study were (I) suction combined with agitation and rotation of a brush, (II) suction, using the central suction system installed in the building, and (III) suction combined with rotation of a brush. Each cleaning system was adapted to utilize special paper bags for collecting the dirt which was removed from the carpets during each day's cleaning. A new paper bag, which had previously been weighed after being conditioned to 65 -percent relative humidity and $70^{\circ} \mathrm{F}$, was used each day. The duration of each daily cleaning was about 45 minutes on $900 \mathrm{ft}^{2}$ of carpet. The actual cleaning time was recorded for each day. The filled bags were again weighed after being conditioned to 65-percent relative humidity and $70^{\circ} \mathrm{F}$., and the weight of the dirt collected each day was computed. The rate of wear of the carpets was determined by changes in the thickness of the pile.

The appearance of the carpets, after beginning the closely supervised cleaning on January 10, 1940, was definitely improved at the end of the first week. The type of cleaning had no measurable effect on the rate of wear of the carpets. Whether this would also be the case if similar experiments were conducted on new carpets cannot be determined from these tests.

The amount of dirt which is collected each day in experiments of this character depends upon a number of factors. It seems worth while to discuss the results more fully to aid those who may undertake similar experiments in the future.

The average amount of dirt collected per day for each of the 27 weeks of this investigation and the standard error of each average are given in table 7. The significance ratios of the difference between successive weeks are also given in the table, together with meteorological data on precipitation, sunshine, and remarks regarding number of days of rain, snow, sleet, and thawing.

The difference between the average amount of dirt collected per day during the first and the second week is highly significant. This difference cannot be attributed to abnormal weather conditions. It is unquestionably due to the removal of dirt which had accumulated in the pile of the carpets over a period of time. The difference between the total amount of dirt collected during the first and the second week is 3 pounds. It may be taken as an estimate of the amount of dirt which had accumulated in the pile of the carpets. On the basis of the daily average for the second week, this accumulated amount would represent approximately the quantity of dirt which is carried onto the carpets during 3 days of service wear. It explains the difference in appearance of the carpets before and after the beginning of the closely supervised cleaning. The cumulative daily amount of dirt removed during the first 4 weeks, Sundays excepted, is plotted in 
TABLE 7.-Results of tests with three vacuum-cleaning systems

\begin{tabular}{|c|c|c|c|c|c|c|}
\hline $\begin{array}{l}\text { Type of } \\
\text { cleaning }\end{array}$ & $\begin{array}{c}\text { Week } \\
\text { number }\end{array}$ & $\begin{array}{l}\text { Daily } \\
\text { average } \\
\text { amount } \\
\text { of dirt } \\
\text { collected } \\
\text { during } \\
\text { week }\end{array}$ & $\begin{array}{c}\text { Significance } \\
\text { ratio of } \\
\text { difference } \\
\text { between } \\
\text { con- } \\
\text { secutive } \\
\text { weeks a }\end{array}$ & $\begin{array}{c}\text { Precipi- } \\
\text { tation }\end{array}$ & Sunshine & Remarks \\
\hline III... & \begin{tabular}{r|}
1 \\
2 \\
3 \\
4 \\
5 \\
6 \\
7 \\
7 \\
8 \\
9 \\
10 \\
11 \\
12 \\
13 \\
14 \\
15 \\
16 \\
17 \\
18 \\
19 \\
20 \\
21 \\
22 \\
23 \\
24 \\
25 \\
26 \\
27
\end{tabular} & $\begin{array}{c}l b . \\
1.45 \pm .06 \\
0.93 \pm .09 \\
.93 \pm .06 \\
.93 \pm .08 \\
.99 \pm .08 \\
1.03 \pm .15 \\
0.76 \pm .07 \\
.78 \pm .06 \\
.54 \pm .06 \\
.66 \pm .08 \\
.59 \pm .04 \\
.58 \pm .04 \\
.59 \pm .02 \\
.75 \pm .03 \\
.58 \pm .07 \\
.56 \pm .03 \\
.46 \pm .05 \\
.59 \pm .04 \\
.61 \pm .09 \\
.60 \pm .05 \\
.47 \pm .04 \\
.54 \pm .03 \\
.63 \pm .04 \\
.63 \pm .05 \\
.60 \pm .06 \\
.48 \pm .02 \\
.53 \pm .04\end{array}$ & $\begin{array}{r}* * 4.7 \\
0.0 \\
.0 \\
.5 \\
.3 \\
1.7 \\
0.2 \\
* 2.9 \\
1.3 \\
0.8 \\
.3 \\
.2 \\
* * 4.0 \\
* 2.4 \\
0.2 \\
1.7 \\
* 2.0 \\
0.2 \\
.1 \\
* 2.0 \\
1.2 \\
1.9 \\
0.0 \\
.3 \\
* 2.1 \\
1.3\end{array}$ & $\begin{array}{r}\text { Ins. } \\
0.70 \\
.15 \\
.91 \\
.08 \\
.27 \\
2.19 \\
0.23 \\
.21 \\
0 \\
1.73 \\
0.48 \\
2.33 \\
0.87 \\
2.99 \\
0 \\
.45 \\
.01 \\
1.39 \\
0.49 \\
.09 \\
0 \\
.02 \\
.70 \\
2.39 \\
0.43 \\
.01 \\
2.60\end{array}$ & $\begin{array}{r}\text { Percent } \\
23 \\
59 \\
80 \\
58 \\
50 \\
40 \\
\\
64 \\
18 \\
18 \\
64 \\
50 \\
58 \\
45 \\
46 \\
15 \\
73 \\
46 \\
67 \\
56 \\
35 \\
68 \\
41 \\
55 \\
55 \\
56 \\
48 \\
67 \\
72\end{array}$ & $\begin{array}{l}\text { Sleet on } 1 \text { day; thawing on } 4 \text { days. } \\
\text { Snow on } 2 \text { days. } \\
\text { Snow on } 1 \text { day, thawing on } 6 \text { days. } \\
\text { Thawing on } 6 \text { days, rain on } 1 \text { day. } \\
\text { Rain on } 1 \text { day. } \\
\text { Snow on } 1 \text { day, sleet on } 2 \text { days, thawing } \\
\text { on } 3 \text { days; rain on } 3 \text { days. } \\
\text { Snow on } 1 \text { day, sleet on } 1 \text { day, thawing } \\
\text { on } 1 \text { day, rain on } 1 \text { day. } \\
\text { Rain on } 5 \text { days. } \\
\text { Snow on } 1 \text { day, rain on } 2 \text { days. } \\
\text { Rain on } 3 \text { days. } \\
\text { Do. } \\
\text { Snow on } 1 \text { day, sleet on } 1 \text { day, rain on } \\
3 \text { days. } \\
\text { Rain on } 6 \text { days. } \\
\text { Rain on } 3 \text { days. } \\
\text { Rain on } 1 \text { day. } \\
\text { Rain on } 4 \text { days. } \\
\text { Rain on } 1 \text { day. } \\
\text { Rain on } 4 \text { days. } \\
\text { Do. } \\
\text { Rain on } 2 \text { days. } \\
\text { Rain on } 1 \text { day. }\end{array}$ \\
\hline
\end{tabular}

- Double and single asterisks indicate highly significant and significant ratios, respectively.

figure 38. The curve approaches a straight line after the first week. The slope of this straight line equals both the average amount of dirt deposited on the carpets each day and the average amount removed each day by the cleaning unit. The ordinate intercept, namely 3 pounds, represents the amount of accumulated dirt which was removed from the carpets by the cleaning unit during the first week and is in addition to the amount which was carried onto the carpets during this week and was also removed.

The random daily average fluctuations for the 27 weeks, table 7 , are attributable to differences in weather conditions. For example, during the sixth week the precipitation was 2.19 inches; the snowfall was 2.7 inches; there was sleet on 2 days; and the sunshine was equal to 40 percent of the possible amount. On the other hand, during the seventh week the precipitation was 0.23 inch; the snowfall was 1.51 inches; there was sleet on 1 day; and the sunshine was equal to 64 percent of the possible amount. Similar differences may be noted between the eighth and ninth weeks, with 5 days of rain for a total precipitation of 1.21 inches and only 18 percent of sunshine during the eighth week compared with no precipitation and 65 percent of sunshine during the ninth week. It is apparent that there is a definite correlation between the amount of dirt deposited on the carpets and the weather conditions.

In addition to the random daily average fluctuations just referred to, systematic changes in the daily average may also be noted. These systematic changes are shown in figure 39 , where the cumulative daily 


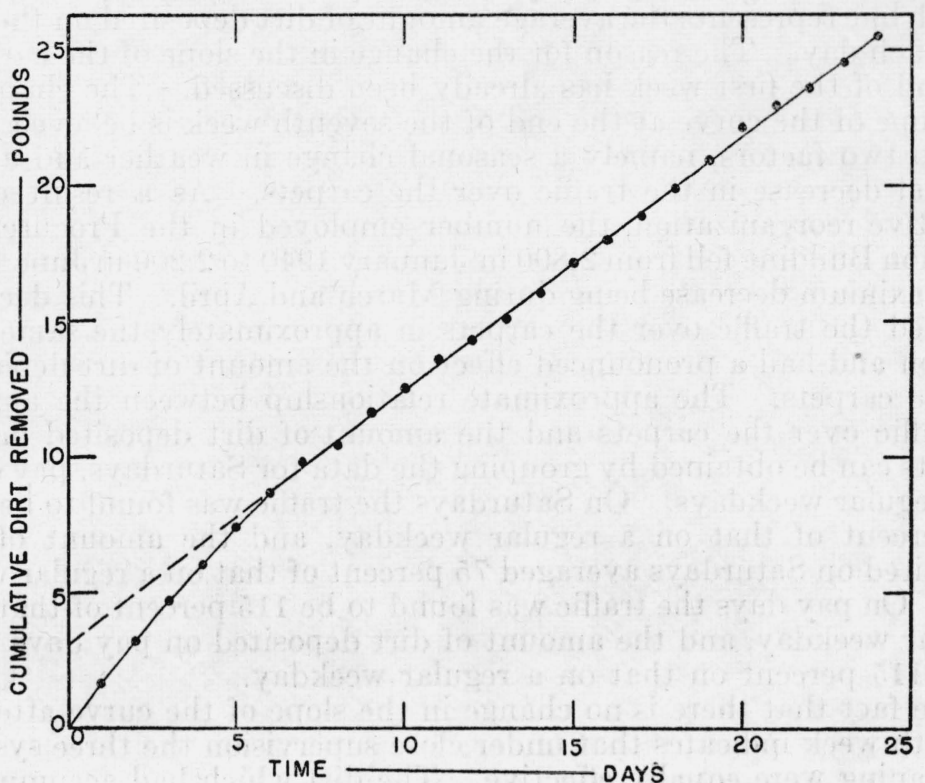

FigURE 38.-Cumulative amount of dirt removed from the carpets per day during 24 days of service wear.

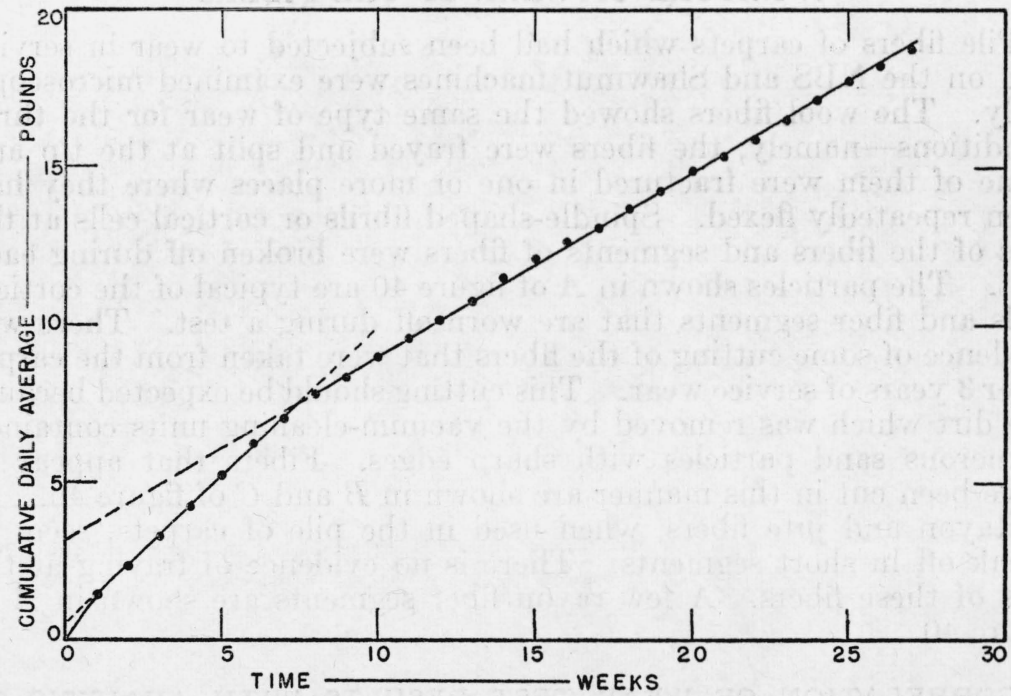

FigURE 39.-Cumulative daily average amount of dirt removed from the carpets per week during 27 weeks of service wear. 
average per week is plotted for the 27 weeks. The curve is comprised of three sections, each being represented by a straight line. The slope of each line represents the average amount of dirt deposited on the carpets each day. The reason for the change in the slope of the curve at the end of the first week has already been discussed. The change in the slope of the curve at the end of the seventh week is believed to be due to two factors, namely a seasonal change in weather and a substantial decrease in the traffic over the carpets. As a result of an executive reorganization, the number employed in the Procurement Division Building fell from 2,800 in January 1940 to 2,200 in June 1940, the maximum decrease being during March and April. This decrease lessened the traffic over the carpets in approximately the same proportion and had a pronounced effect on the amount of dirt deposited on the carpets. The approximate relationship between the amount of traffic over the carpets and the amount of dirt deposited on the carpets can be obtained by grouping the data for Saturdays, pay days, and regular weekdays. On Saturdays the traffic was found to be only 60 percent of that on a regular weekday, and the amount of dirt deposited on Saturdays averaged 75 percent of that on a regular weekday. On pay days the traffic was found to be 115 percent of that on a regular weekday, and the amount of dirt deposited on pay days averaged 115 percent on that on a regular weekday.

The fact that there is no change in the slope of the curve after the seventh week indicates that under close supervision the three systems of cleaning were equally effective. The dirt which had accumulated in the pile of the carpets prior to the close supervision in the use of the three cleaning systems is not attributable to the inadequacy of the cleaning system that was used but to the inadequate operation of the system.

\section{NATURE OF WEAR OF THE FIBERS}

Pile fibers of carpets which had been subjected to wear in service and on the NBS and Shawmut machines were examined microscopically. The wool fibers showed the same type of wear for the three conditions-namely, the fibers were frayed and split at the tip and some of them were fractured in one or more places where they had been repeatedly flexed. Spindle-shaped fibrils or cortical cells at the tips of the fibers and segments of fibers were broken off during each test. The particles shown in $A$ of figure 40 are typical of the cortical cells and fiber segments that are worn off during a test. There was evidence of some cutting of the fibers that were taken from the carpet after 3 years of service wear. This cutting should be expected because the dirt which was removed by the vacuum-cleaning units contained numerous sand particles with sharp edges. Fibers that appear to have been cut in this manner are shown in $B$ and $C$ of figure 40.

Rayon and jute fibers, when used in the pile of carpets, seem to break off in short segments. There is no evidence of fraying at the tips of these fibers. A few rayon fiber segments are shown in $A$ of figure 40 .

\section{CORRELATION OF WEAR TEST RESULTS WITH ANALYSIS OF CARPETS}

The product of pile density squared and height of pile, $D^{2} H$, is plotted in figure 41 against the $S$ index obtained with the NBS 


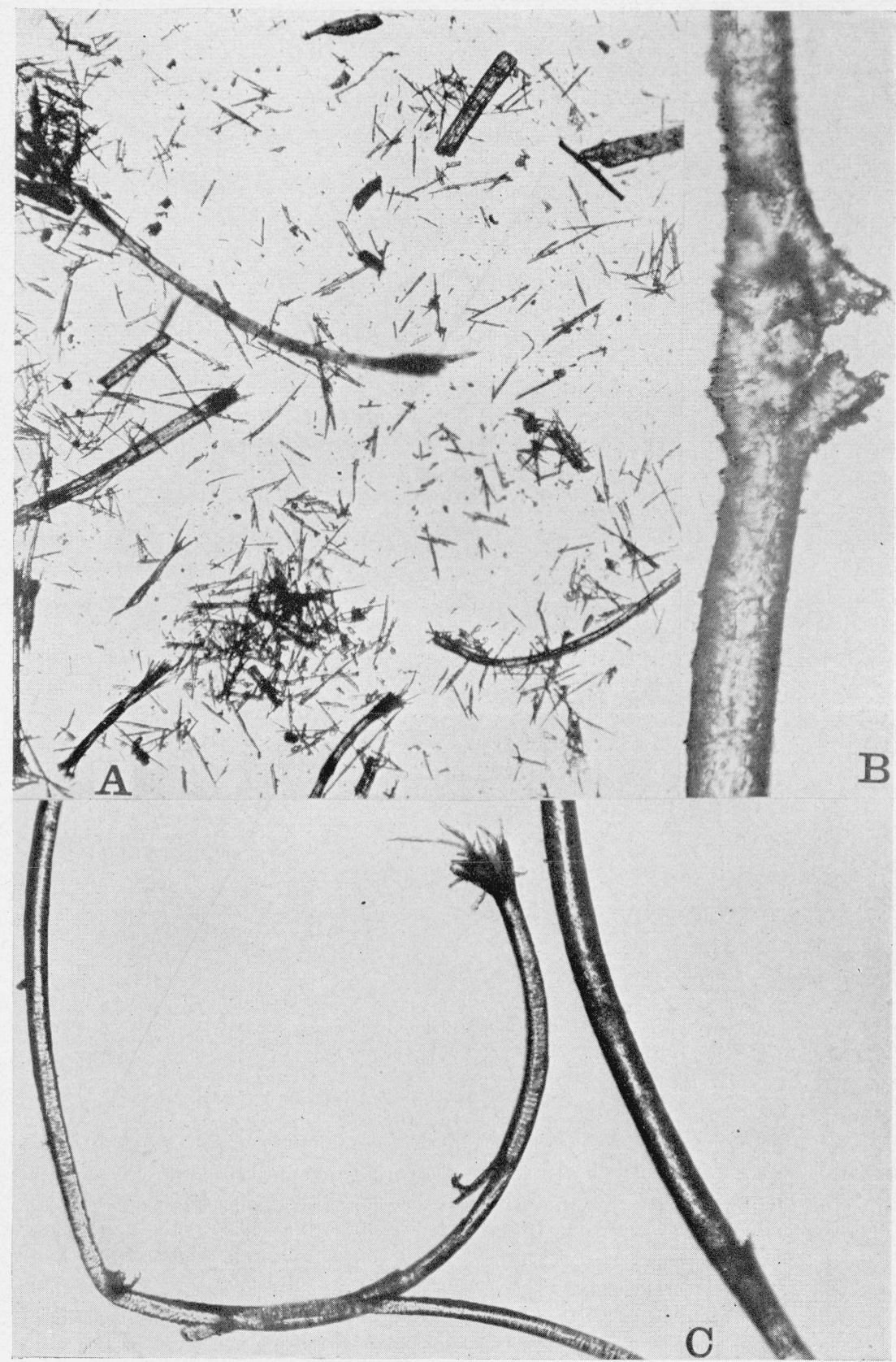

FIGURE 40.-Nature of wear on fibers.

$A$, Wool and rayon fibers worn on Shawmut machine. Magnification about $\times 50$.

$B$, Wool fiber worn 3 years in the service test. Magnification about $\times 275$.

$C$, Wool fiber worn 3 years in the service test. Magnification about $\times 100$. 
and Shawmut machines, respectively. The points belonging to the same series of carpets are connected by straight lines. It is apparent that the $S$ index and $D^{2} H$ for each series of carpets show in general a good linear correlation. However, such factors as weave, blend of wool, and nature of the pile affect the correlation

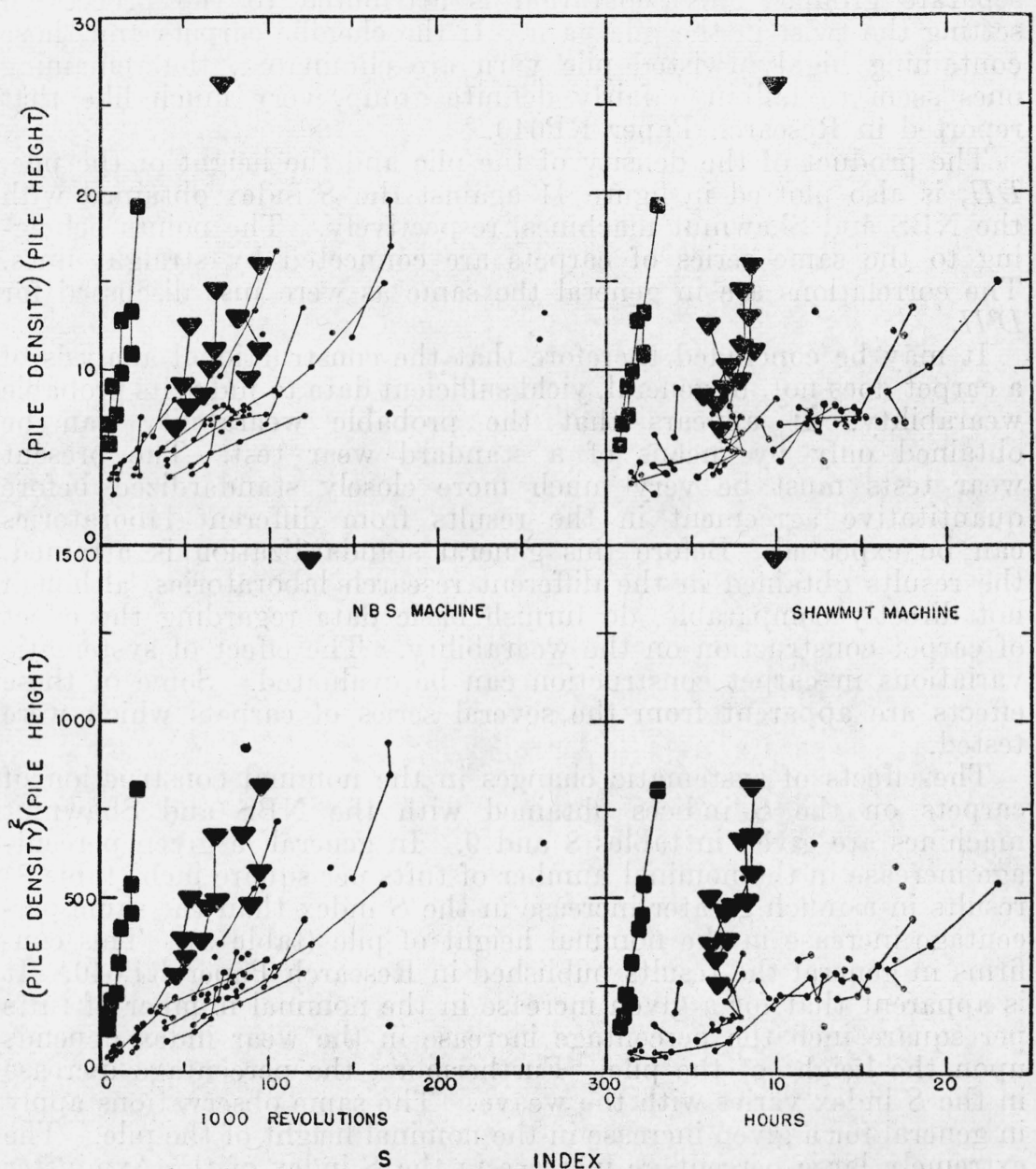

FIGURE 41.- Relationship between the $S$ indices of all carpets tested and the products of $D^{2} H$ and $D H$, where $D$ and $H$ are density and height of pile, respectively.

The chenile carpets are represented by the solid squares and the carpets containing highly twisted pile are represented by the solid triangles.

greatly. The chenille carpets are definitely isolated from the carpets of the other weaves. The very low wear indices for the chenille carpets were not expected because of the reputed long wear which chenille carpets give in service. The alkali-solubility of the wool forming the pile of these carpets was determined according to the method of Harris and $\operatorname{Smith}^{8}$ and was found to be 80 percent for

${ }^{8}$ Milton Harris and Arthur L. Smith, Oxidation of wool: Alkali-solubility test for determining the extent of oxidation, J. Research NBS 17, 577-583 (1936) RP928. 
each carpet. Apparently the wool used in weaving these carpets was seriously damaged during processing. The very high alkalisolubility* explains fully the low wear indices which were obtained and also the separation of these carpets from the others in figure 41. The carpets in which the pile yarns are highly twisted also fall in a separate group. This separation is attributed to the process of setting the twist in the pile yarn. If the chenille carpets and those containing highly twisted pile yarn are eliminated, the remaining ones seem to fall in a fairly definite group, very much like that reported in Research Paper RP640. ${ }^{9}$

The product of the density of the pile and the height of the pile, $D H$, is also plotted in figure 41 against the $S$ index obtained with the NBS and Shawmut machines, respectively. The points belonging to the same series of carpets are connected by straight lines. The correlations are in general the same as were just discussed for $D^{2} H$.

It may be concluded therefore that the constructional analysis of a carpet does not, in general, yield sufficient data to judge its probable wearability. It appears that the probable wearability can be obtained only by means of a standard wear test. The present wear tests must be very much more closely standardized before quantitative agreement in the results from different laboratories can be expected. Before this general standardization is attained, the results obtained in the different research laboratories, although not directly comparable, do furnish basic data regarding the effect of carpet construction on the wearability. The effect of systematic variations in carpet construction can be evaluated. Some of these effects are apparent from the several series of carpets which were tested.

The effects of systematic changes in the nominal construction of carpets on the $S$ indices obtained with the NBS and Shawmut machines are given in tables 8 and 9 . In general, a given percentage increase in the nominal number of tufts per square inch (table 8) results in a much greater increase in the $S$ index than the same percentage increase in the nominal height of pile (table 9). This confirms in general the results published in Research Paper RP640. It is apparent that for a given increase in the nominal number of tufts per square inch the percentage increase in the wear index depends upon the height of the pile. Furthermore, the percentage increase in the $S$ index varies with the weave. The same observations apply in general for a given increase in the nominal height of the pile. The extremely large percentage increase in the $S$ index of the Axminster carpets with an increase in the nominal number of tufts per square inch results partly because of the decrease in the back thickness of the Axminster carpets having few rows per inch. No correction has been made in these tests for this decrease in back thickness of the Axminster carpets.

Another series of carpets which is of interest consists of six velvet carpets in which a stock-dyed standard base blend of the pile wool was combined with 20 percent of different wool wastes. The percentage changes in the $S$ indices obtained with the NBS and Shawmut

\footnotetext{
- Herbert F. Schiefer and Richard S. Cleveland, Wear of carpets, BS J. Research 12, 155-166 (1934) RP640. *The alkali-solubility of the wool forming the pile of carpets of commercial production ordinarily does not exceed about 20 percent.
} 
machines are given in table 10 . The utilization of 20 percent of these respective wool wastes with the standard base blend had in general a beneficial rather than a detrimental effect on the $S$ index.

TABLE 8.-Effect of systematic changes in the nominal construction of carpets on the $S$ indices

[The increase in $S$ index is computed for systematic increases in tufts per square inch, rows times pitch]

\begin{tabular}{|c|c|c|c|c|c|c|}
\hline \multicolumn{3}{|c|}{ Nominal construction of carpets } & \multirow{2}{*}{$\begin{array}{l}\text { Nominal } \\
\text { increase } \\
\text { in tufts } \\
\text { per square } \\
\text { inch }\end{array}$} & \multicolumn{3}{|c|}{ Increase in $S$ index } \\
\hline $\begin{array}{l}\text { Wire } \\
\text { height }\end{array}$ & $\begin{array}{l}\text { Pitch per } \\
\text { inch }\end{array}$ & $\begin{array}{l}\text { Rows per } \\
\text { inch }\end{array}$ & & $\begin{array}{l}\text { NBS } \\
\text { machine }\end{array}$ & $\begin{array}{l}\text { Shawmut } \\
\text { machine }\end{array}$ & Service \\
\hline \multicolumn{7}{|c|}{ WILTON } \\
\hline Inch & & & Percent & Percent & Percent & Percent \\
\hline 0.145 & 8 & $\begin{array}{r}10 \\
10 \\
12 \\
8\end{array}$ & $\begin{array}{l}25 \\
50\end{array}$ & $\begin{array}{r}65 \\
154\end{array}$ & $\begin{array}{l}26 \\
58\end{array}$ & - \\
\hline .172 & 8 & $\begin{array}{l}10 \\
10 \\
12\end{array}$ & 50 & $\begin{array}{l}46 \\
98\end{array}$ & $\begin{array}{l}22 \\
57\end{array}$ & . \\
\hline .145 & 9.5 & $\begin{array}{l}0 \\
10 \\
12\end{array}$ & $\begin{array}{l}25 \\
50\end{array}$ & $\begin{array}{l}100 \\
152\end{array}$ & $\begin{array}{l}18 \\
45\end{array}$ & $\begin{array}{l}50 \\
81\end{array}$ \\
\hline \multicolumn{7}{|c|}{${ }^{*}$ CHENILLE } \\
\hline & 10 & 5 & & & & \\
\hline a 0.625 & $\begin{array}{l}12 \\
14 \\
10\end{array}$ & $\begin{array}{l}6 \\
7 \\
5\end{array}$ & $\begin{array}{l}44 \\
96\end{array}$ & $\begin{array}{l}100 \\
317\end{array}$ & $\begin{array}{r}57 \\
200\end{array}$ & 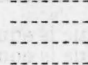 \\
\hline s. 75 & $\begin{array}{l}10 \\
14 \\
10\end{array}$ & $\begin{array}{l}6 \\
7 \\
5\end{array}$ & $\begin{array}{l}44 \\
96\end{array}$ & $\begin{array}{l}109 \\
185\end{array}$ & $\begin{array}{r}50 \\
110\end{array}$ & - \\
\hline a 1.00 & $\begin{array}{l}10 \\
12 \\
14\end{array}$ & $\begin{array}{l}5 \\
7 \\
7\end{array}$ & $\begin{array}{l}44 \\
96\end{array}$ & $\begin{array}{l}102 \\
275\end{array}$ & $\begin{array}{r}46 \\
131\end{array}$ & - \\
\hline \multicolumn{7}{|c|}{ AXMINSTER } \\
\hline b 0.625 & 7 & $\left\{\begin{array}{l}4.67 \\
5.5 \\
7.25 \\
9 \\
4.67\end{array}\right.$ & $\begin{array}{l}18 \\
55 \\
93\end{array}$ & $\begin{array}{r}27 \\
222 \\
358\end{array}$ & $\begin{array}{r}-45 \\
42 \\
46\end{array}$ & - \\
\hline b. 875 & 7 & $\begin{array}{l}5.5 \\
7.25 \\
9 \\
4\end{array}$ & $\begin{array}{l}18 \\
55 \\
93\end{array}$ & $\begin{array}{r}79 \\
261 \\
780\end{array}$ & $\begin{array}{r}48 \\
108 \\
211\end{array}$ & (1) \\
\hline b. 875 & 7 & $\begin{array}{l}4.25 \\
4.25 \\
4.75 \\
6 \\
8\end{array}$ & $\begin{array}{r}6 \\
12 \\
19 \\
50 \\
100\end{array}$ & $\begin{array}{r}43 \\
57 \\
107 \\
475 \\
1,150\end{array}$ & $\begin{array}{r}133 \\
87 \\
153 \\
380 \\
667\end{array}$ & $\begin{array}{r}8 \\
23 \\
57 \\
134 \\
300\end{array}$ \\
\hline \multicolumn{7}{|c|}{ PLAIN VELVET } \\
\hline 0.18 & $\begin{array}{l}7.25 \\
8\end{array}$ & $\begin{array}{l}8.8 \\
8.8\end{array}$ & 10 & 30 & 30 & (n).......... \\
\hline .22 & $\begin{array}{l}7.25 \\
8\end{array}$ & $\begin{array}{l}8.8 \\
8.8\end{array}$ & 10 & 29 & 12 & (n) \\
\hline .28 & $\begin{array}{l}7.25 \\
8\end{array}$ & $\begin{array}{l}8.8 \\
8.8\end{array}$ & 10 & 22 & 23 & 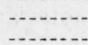 \\
\hline .41 & $\begin{array}{l}7.25 \\
8\end{array}$ & $\begin{array}{l}8.8 \\
8.8\end{array}$ & 10 & 39 & 28 & 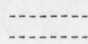 \\
\hline .187 & 8 & $\begin{array}{r}0 \\
7 \\
8 \\
9 \\
10\end{array}$ & $\begin{array}{l}17 \\
33 \\
50 \\
67\end{array}$ & $\begin{array}{r}33 \\
62 \\
103 \\
199\end{array}$ & $\begin{array}{l}17 \\
49 \\
32 \\
51\end{array}$ & $\begin{array}{r}54 \\
56 \\
103 \\
109\end{array}$ \\
\hline
\end{tabular}

See footnotes at end of table. 
TABLE 8.-Effect of systematic changes in the nominal construction of carpets on the $S$ indices-Continued

\begin{tabular}{|c|c|c|c|c|c|c|}
\hline \multicolumn{3}{|c|}{ Nominal construction of carpets } & \multirow{2}{*}{$\begin{array}{c}\text { Nominal } \\
\text { increase } \\
\text { in tufts } \\
\text { per square } \\
\text { inch }\end{array}$} & \multicolumn{3}{|c|}{ Increase in $S$ index } \\
\hline $\begin{array}{l}\text { Wire } \\
\text { height }\end{array}$ & $\begin{array}{l}\text { Pitch per } \\
\text { inch }\end{array}$ & $\begin{array}{l}\text { Rows per } \\
\text { inch }\end{array}$ & & $\begin{array}{c}\text { NBS } \\
\text { machine }\end{array}$ & $\begin{array}{l}\text { Shawmut } \\
\text { machine }\end{array}$ & Service \\
\hline \multicolumn{7}{|c|}{ HARD TWIST VELVET } \\
\hline Inch & & & Percent & Percent & Percent & Perecnt \\
\hline 0.145 & 7.67 & $\begin{array}{l}8.5 \\
9.5\end{array}$ & $\begin{array}{l}13 \\
27\end{array}$ & $\begin{array}{r}41 \\
104\end{array}$ & $\begin{array}{r}c-9 \\
13\end{array}$ & (n) \\
\hline .192 & 7.67 & $\begin{array}{l}8.5 \\
9.5\end{array}$ & $\begin{array}{l}13 \\
27\end{array}$ & $\begin{array}{l}32 \\
90\end{array}$ & $\begin{array}{l}13 \\
30\end{array}$ & . \\
\hline & 7.67 & $\begin{array}{l}8.5 \\
9.5\end{array}$ & $\begin{array}{l}13 \\
27\end{array}$ & $\begin{array}{l}38 \\
75\end{array}$ & $\begin{array}{l}16 \\
18\end{array}$ & .......... \\
\hline .290 & 7.67 & $\begin{array}{l}8.5 \\
9.5\end{array}$ & $\begin{array}{l}13 \\
27\end{array}$ & $\begin{array}{l}28 \\
79\end{array}$ & $\begin{array}{l}33 \\
41\end{array}$ & (2) \\
\hline \multicolumn{7}{|c|}{ PRINTED VELVET } \\
\hline 0.25 & 8 & $\begin{array}{l}7 \\
9\end{array}$ & 29 & 111 & & (n........ \\
\hline .37 & 8 & 9 & 29 & 86 & & (n...... \\
\hline .44 & 8 & 9 & 29 & 64 & & (n.......... \\
\hline
\end{tabular}

a This value is equal to the nominal length of the tuft or fur.

b This value is equal to the nominal length of the tuft or finish cut.

- A negative value indicates a decrease in $S$ index.

- These chenille carpets were not typical of commercial production.

\section{TABLE 9.-Effect of systematic changes in the nominal construction of carpets on the} $S$ indices

[The increase in $S$ index is computed for systematic increases in wire height]

\begin{tabular}{|c|c|c|c|c|c|c|}
\hline \multicolumn{3}{|c|}{ Nominal construction of carpets } & \multirow{2}{*}{$\begin{array}{c}\text { Nominal } \\
\text { increase in } \\
\text { height of } \\
\text { of pile }\end{array}$} & \multicolumn{3}{|c|}{ Increase in!S index } \\
\hline $\begin{array}{l}\text { Rows } \\
\text { per inch }\end{array}$ & $\begin{array}{l}\text { Pitch per } \\
\text { inch }\end{array}$ & $\begin{array}{c}\text { Wire } \\
\text { height }\end{array}$ & & $\begin{array}{l}\text { NBS } \\
\text { machine }\end{array}$ & $\begin{array}{l}\text { Shawmut } \\
\text { machine }\end{array}$ & Service \\
\hline \multicolumn{7}{|c|}{ WILTON } \\
\hline \multirow[b]{2}{*}{8} & \multirow[b]{2}{*}{8} & \multirow{6}{*}{$\begin{array}{r}\text { Inches } \\
0.145 \\
.172 \\
.145 \\
.172 \\
.145 \\
.172 \\
.145 \\
.175 \\
.205\end{array}$} & Percent & Percent & Percent & Percent \\
\hline & & & 19 & 25 & -4 & (n) \\
\hline 10 & 8 & & 19 & 11 & $a-7$ & (n)........... \\
\hline 12 & 8 & & $19^{-\cdots}$ & $8-3$ & -6 & \\
\hline & & & & & & \\
\hline 10 & 9.5 & & $\begin{array}{l}21 \\
41\end{array}$ & $\begin{array}{l}\mathrm{a}-9 \\
\mathrm{a}-6\end{array}$ & $\begin{array}{l}4 \\
7\end{array}$ & $\begin{array}{l}19 \\
29\end{array}$ \\
\hline
\end{tabular}

See footnotes at end of table. 
TABLE 9.-Effect of systematic changes in the nominal construction of carpets on the $S$ indices-Continued

\begin{tabular}{|c|c|c|c|c|c|c|}
\hline \multicolumn{3}{|c|}{ Nominal construction of carpets } & \multirow{2}{*}{$\begin{array}{l}\text { Nominal } \\
\text { increase in } \\
\text { height of } \\
\text { of pile }\end{array}$} & \multicolumn{3}{|c|}{ Increase in $S$ index } \\
\hline $\begin{array}{c}\text { Rows } \\
\text { per inch }\end{array}$ & $\begin{array}{l}\text { Pitch per } \\
\text { inch }\end{array}$ & $\begin{array}{l}\text { Wire } \\
\text { height }\end{array}$ & & $\begin{array}{c}\text { NBS } \\
\text { mashine }\end{array}$ & $\begin{array}{l}\text { Shawmut } \\
\text { machine }\end{array}$ & Service \\
\hline \multicolumn{7}{|c|}{${ }^{*} \mathrm{CHENILLE}$} \\
\hline & & $\begin{array}{r}\text { Inches } \\
\text { b. } 625\end{array}$ & Percent & Percent & Percent & Percent \\
\hline 5 & 10 & $\begin{array}{l}\text { b. } 75 \\
\text { b. } 0 \\
\text { b0. } 625\end{array}$ & $\begin{array}{l}22 \\
67\end{array}$ & $\begin{array}{l}19 \\
28\end{array}$ & $\begin{array}{l}43 \\
86\end{array}$ & ... \\
\hline 6 & 12 & $\begin{array}{l}\text { b. } 75 \\
\text { b1. } 0 \\
\text { b. } 625\end{array}$ & $\begin{array}{l}22 \\
67\end{array}$ & $\begin{array}{l}24 \\
29\end{array}$ & $\begin{array}{l}36 \\
73\end{array}$ & (-. \\
\hline 7 & 14 & $\begin{array}{l}\text { b. } 75 \\
\text { b1. } 0\end{array}$ & $\begin{array}{l}22 \\
67\end{array}$ & $\begin{array}{r}-5 \\
14\end{array}$ & $\begin{array}{r}0 \\
42\end{array}$ & 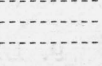 \\
\hline \multicolumn{7}{|c|}{ AXMINSTER } \\
\hline 4.67 & 7 & $\begin{array}{l}c 0.625 \\
0.875\end{array}$ & & & & \\
\hline 5.5 & 7 & $\begin{array}{l}.875 \\
.625\end{array}$ & 42 & 4 & a -51 & \\
\hline & 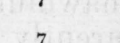 & $\begin{array}{l}\text { c. } 875 \\
\text { c. } 625\end{array}$ & 42 & 47 & 33 & - \\
\hline 7.25 & 7 & $\begin{array}{l}.875 \\
.625\end{array}$ & 42 & 17 & -28 & \\
\hline 9 & 7 & $\begin{array}{l}.027 \\
0.875 \\
0.625\end{array}$ & 42 & 33 & 5 & \\
\hline 6 & 7 & $\begin{array}{r}0.875 \\
01.125\end{array}$ & $\begin{array}{l}42 \\
83\end{array}$ & $\begin{array}{l}1 \\
6\end{array}$ & $\begin{array}{l}13 \\
23\end{array}$ & $\begin{array}{l}33 \\
93\end{array}$ \\
\hline \multicolumn{7}{|c|}{ PLAIN VELVET } \\
\hline 8.8 & 7.25 & $\begin{array}{l}0.18 \\
.22 \\
.28 \\
.41 \\
.18\end{array}$ & $\begin{array}{r}22 \\
56 \\
128\end{array}$ & $\begin{array}{r}8 \\
37 \\
62\end{array}$ & $\begin{array}{l}10 \\
15 \\
33\end{array}$ & \\
\hline 8.8 & 8.0 & $\begin{array}{l}.22 \\
.28 \\
.41 \\
.125\end{array}$ & $\begin{array}{r}22 \\
56 \\
128\end{array}$ & $\begin{array}{r}8 \\
29 \\
74\end{array}$ & $\begin{array}{r}8-6 \\
9 \\
31\end{array}$ & - \\
\hline 8 & 8 & $\begin{array}{l}.164 \\
.187 \\
.247 \\
.290 \\
.375\end{array}$ & $\begin{array}{r}31 \\
50 \\
98 \\
134 \\
200\end{array}$ & $\begin{array}{l}14 \\
20 \\
28 \\
39 \\
49\end{array}$ & $\begin{array}{r}6 \\
22 \\
14 \\
12 \\
23\end{array}$ & $\begin{array}{r}16 \\
22 \\
71 \\
100 \\
117\end{array}$ \\
\hline \multicolumn{7}{|c|}{ HARD TWIST VELVET } \\
\hline 7.5 & 7.67 & $\begin{array}{r}0.145 \\
.192 \\
.246 \\
.290 \\
.145\end{array}$ & $\begin{array}{r}32 \\
70 \\
100\end{array}$ & $\begin{array}{l}16 \\
13 \\
24\end{array}$ & $\begin{array}{r}\mathrm{a}-6 \\
4 \\
\mathrm{a}-10\end{array}$ & $\begin{array}{l}\cdots \\
\cdots \\
\cdots\end{array}$ \\
\hline 8.5 & 7.67 & $\begin{array}{l}.192 \\
.246 \\
.290 \\
.145\end{array}$ & $\begin{array}{r}32 \\
70 \\
100\end{array}$ & $\begin{array}{r}9 \\
11 \\
12\end{array}$ & $\begin{array}{l}16 \\
32 \\
31\end{array}$ & - \\
\hline 9.5 & 7. 67 & $\begin{array}{l}.192 \\
.246 \\
.290\end{array}$ & $\begin{array}{r}32 \\
70 \\
100\end{array}$ & $\begin{array}{r}8 \\
-3 \\
8\end{array}$ & $\begin{array}{r}8 \\
9 \\
12\end{array}$ & - \\
\hline \multicolumn{7}{|c|}{ PRINTED VELVET } \\
\hline 7 & 8 & $\begin{array}{r}0.250 \\
.370 \\
.440 \\
.250\end{array}$ & $\begin{array}{l}48 \\
76\end{array}$ & $\begin{array}{l}30 \\
48\end{array}$ & & - \\
\hline 9 & 8 & $\begin{array}{l}.370 \\
.440\end{array}$ & $\begin{array}{l}48 \\
76\end{array}$ & $\begin{array}{l}15 \\
15\end{array}$ & & - \\
\hline
\end{tabular}

a A negative value indicates a decrease in $S$ index.

b This value is equal to the nominal length of the tuft or fur.

c This value is equal to the nominal length of the tuft or finish eut.

* These chenille carpets were not typical of commercial production. 
TABLE 10.-Effect of the admixture of various wool wastes with the standard base blend of the pile wool on the $S$ index

\begin{tabular}{|c|c|c|}
\hline \multirow{2}{*}{ Composition of pile wool } & \multicolumn{2}{|c|}{$\begin{array}{l}\text { Change in } S \text { index based } \\
\text { upon the } S \text { index of the } \\
\text { standard base blend }\end{array}$} \\
\hline & $\begin{array}{c}\text { NBS } \\
\text { machine }\end{array}$ & $\begin{array}{l}\text { Shawmut } \\
\text { machine }\end{array}$ \\
\hline $\begin{array}{l}\text { Standard base blend plus } 20 \text { percent of dusted droppings } \\
\text { Standard base blend plus } 20 \text { percent of garnetted threads } \\
\text { Standard base blend plus } 20 \text { percent of blend through Schofield Picker } \\
\text { Standard base blend plus } 20 \text { percent of dusted droppings carded on breaker card.- } \\
\text { Standard base blend plus } 20 \text { percent of roving waste of base blend }\end{array}$ & $\begin{array}{r}\text { Percent } \\
\text { a } 5 \\
36 \\
20 \\
30 \\
32\end{array}$ & $\begin{array}{r}\text { Percent } \\
17 \\
42 \\
35 \\
49 \\
32\end{array}$ \\
\hline
\end{tabular}

a A negative value indicates a decrease in $S$ index.

\section{LIMITATIONS OF WEAR-TESTING MACHINES}

The Shawmut machine was not found suitable for testing all pilefaced carpets. This was especially true for printed velvet carpets and for carpets where the pile yarn contained very little extractable oils. When these carpets were tested on the Shawmut machine, the wearing surface became excessively heated, apparently because of the higher friction between the wearing surface and the pile of the carpets. As the wearing surface became heated, the moisture content of the fibers was reduced and the friction between the pile and wearing surface was increased. This condition usually produced a shimmy motion between the wearing surface and the carpet specimen, and the fibers of the pile were pulled out in a short time. Figure 9 illustrates the general appearance of the specimen after such a test, and figure 42 shows the poor correlation which is obtained between the results of the NBS and Shawmut machines if the results of these tests are included. Intermittent operation of the Shawmut machine to minimize the amount of heating of the wearing surface or the use of a cooling device did not eliminate this type of wear. No difficulty was encountered in testing these carpets with the NBS machine, and no heating of the wearing surface was observed. The automatic adjustment of the speeds of the turntable and braking pulley relative to the driving pulley of the NBS machine, when the friction between the pile and the wearing surface is increased, prevents excessive generation of heat during the test. In this respect the friction drive of the NBS machine is preferable to the positive drive of the Shawmut machine.

The NBS machine has been found suitable for testing all types of pile-faced carpets. Probably the chief limitation of this machine has been the difficulty of obtaining a constant wearing surface. This difficulty has been overcome to a great degree by the specification for the leather and the procedure of preparing the wearing surface, which have been developed by Section IV on Pile Floor Coverings, of Subcommittee A-3, Committee D-13, ASTM. The matter of maintaining a constant wearing surface is still a serious problem and depends largely upon the care given to the machine by the operator. The inherent variation between different pieces of leather cannot be eliminated and ultimately may require the use of some other material for the wearing surface of this machine. The lack of uniform design of the NBS-type machines which are being used probably accounts 
for some of the systematic differences in the results obtained in the interlaboratory tests.

The decrease in the back thickness of low-quality Axminster carpets during a test with the NBS machine presents a difficult problem for evaluating their relative wear resistances. Although this same condition was also encountered in the service tests of the low-quality Axminster carpets, further work needs to be done to obtain the correct solution. The lack of standardization of the NBS machine in the different laboratories can be rectified and uniform procedures for testing and for the evaluation of the wear index should be established

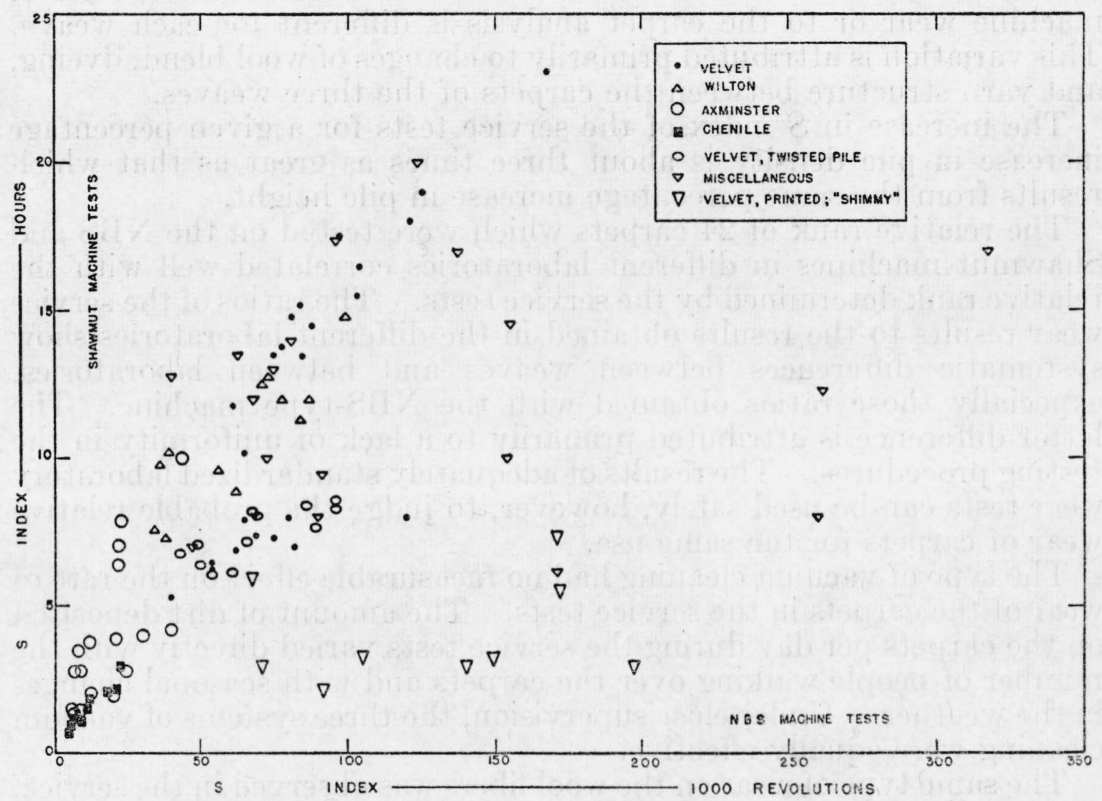

FIGURE 42.-Relationship between the $S$ indices of all carpets tested with the NBS and Shawmut machines.

as soon as possible. The results presented in this paper should be of value in this standardization and unification of carpet wear testing.

\section{SUMMARY}

The NBS and Shawmut machines for testing the wear resistance of carpets, a gage for measuring the thickness of carpets, and an instrument for measuring the length of a tuft of pile from a carpet are described.

The results of the tests with the NBS machine depend upon the downward force at each pulley, the braking torque, the speed of the turntable, the height of the nozzle above the pile, the amount of suction at the nozzle, and the relative humidity and temperature of the air in which the tests are made. These factors must be maintained constant in order to effect standardization of the wear test and to obtain quantitative agreement in the results between different laboratories. 
The $S$ index, which is the time required to wear the pile of a carpet down to one-fourth of the matted pile thickness, is more reliable than the $K$ and $E$ indices, and in the service tests it corresponded more nearly with the time at which a carpet would be discarded on account of visible wear. The deviation of the $S$ index of a single test from the average is less than 10 percent in 9 trials out of 10 .

The correlation of the results obtained during the 3 years of severe wear in service on 24 carpets of Axminster, velvet, and Wilton weaves with the results obtained with the NBS and Shawmut machines on the same carpets and with the analysis of the carpets is good for each weave. However, the ratio of the results of service wear to the results of machine wear or to the carpet analysis is different for each weave. This variation is attributed primarily to changes of wool blend, dyeing, and yarn structure between the carpets of the three weaves.

The increase in $S$ index of the service tests for a given percentage increase in pile density is about three times as great as that which results from the same percentage increase in pile height.

The relative rank of 24 carpets which were tested on the NBS and Shawmut machines in different laboratories correlated well with the relative rank determined by the service tests. The ratios of the service wear results to the results obtained in the different laboratories show systematic differences between weaves and between laboratories, expecially those ratios obtained with the NBS-type machine. The latter difference is attributed primarily to a lack of uniformity in the testing procedures. The results of adequately standardized laboratory wear tests can be used safely, 'however, to judge the probable relative wear of carpets for the same use.

The type of vacuum cleaning had no measurable effect on the rate of wear of the carpets in the service tests. The amount of dirt deposited on the carpets per day during the service tests varied directly with the number of people walking over the carpets and with seasonal changes in the weather. Under close supervision, the three systems of vacuum cleaning were equally effective.

The same type of wear on the wool fibers was observed in the service, NBS, and Shawmut machine wear tests. The fibers were frayed and split at the tip and some of them were fractured in one or more places. Spindle-shaped fibrils or cortical cells were broken off at the tips of the fibers or segments of fibers were broken off. Some of the fibers were partially cut by the sharp edges of sand particles which were deposited in the pile during the service tests. Jute and rayon pile fibers seem to break off in short segments during a wear test, with no fraying at the tips of the fibers.

The correlation between the results of wear tests and the constructional analyses of carpets depends upon such factors as weave, blend of wool, dyeing, chemical degradation of the wool, and twist of the pile yarn. The constructional analysis of a carpet does not yield sufficient data in general to judge the probable wearability of the carpet.

A given increase in the nominal number of tufts per square inch, density of pile, increases the $S$ index very much more than the same percentage increase in nominal height of pile and confirms the results published in NBS Research Paper RP640. The increase in $S$ index of a carpet for a given increase in pile height and pile density varies with the type of wear test and also with weave and pile construction. 
The author expresses his appreciation to A. G. Ashcroft, J. N. Dow, G. E. Hopkins, L. R. Jones, and A. G. Mann for their interest and suggestions during this investigation. The assistance of the Alexander Smith \& Sons Carpet Co., the Bigelow Sanford Carpet Co., the Mohawk Carpet Mills, Inc., the United States Testing Co., the Hoover Co., the Westinghouse Electric \& Mfg. Co., the Shawmut Engineering Co., and the Procurement Division of the United States Treasury Department is gratefully acknowledged.

Washington, September 18, 1942. 